\title{
Data Package of Results from Salt Spill Testing
}

Chemical and Fuel Cycle Technologies Division, Argonne National Laboratory 


\begin{abstract}
About Argonne National Laboratory
Argonne is a U.S. Department of Energy laboratory managed by UChicago Argonne, LLC under contract DE-AC02-06CH11357. The Laboratory's main facility is outside Chicago, at 9700 South Cass Avenue, Argonne, Illinois 60439. For information about Argonne and its pioneering science and technology programs, see www.anl.gov.
\end{abstract}

\title{
DOCUMENT AVAILABILITY \\ Online Access: U.S. Department of Energy (DOE) reports produced after 1991 and a growing number of pre-1991 documents are available free at OSTI.GOV (http://www.osti.gov/), a service of the US Dept. of Energy's Office of Scientific and Technical Information.
}

\author{
Reports not in digital format may be purchased by the public from the \\ National Technical Information Service (NTIS): \\ U.S. Department of Commerce \\ National Technical Information Service \\ 5301 Shawnee Rd \\ Alexandria, VA 22312 \\ www.ntis.gov \\ Phone: (800) 553-NTIS (6847) or (703) 605-6000 \\ Fax: (703) 605-6900 \\ Email: orders@ntis.gov
}

Reports not in digital format are available to DOE and DOE contractors from the Office of Scientific and Technical Information (OSTI):

U.S. Department of Energy

Office of Scientific and Technical Information

P.O. Box 62

Oak Ridge, TN 37831-0062

www.osti.gov

Phone: (865) 576-8401

Fax: (865) 576-5728

Email: reports@osti.gov

\section{Disclaimer}

This report was prepared as an account of work sponsored by an agency of the United States Government. Neither the United States Government nor any agency thereof, nor UChicago Argonne, LLC, nor any of their employees or officers, makes any warranty, express or implied, or assumes any legal liability or responsibility for the accuracy, completeness, or usefulness of any information, apparatus, product, or process disclosed, or represents that its use would not infringe privately owned rights. Reference herein to any specific commercial product, process, or service by trade name, trademark, manufacturer, or otherwise, does not necessarily constitute or imply its endorsement, recommendation, or favoring by the United States Government or any agency thereof. The views and opinions of document authors expressed herein do not necessarily state or reflect those of the United States Government or any agency thereof, Argonne National Laboratory, or UChicago Argonne, LLC. 


\section{Data Package of Results from Salt Spill Testing}

Prepared by

Sara Thomas and Josh Jackson

Chemical and Fuel Cycle Technologies Division, Argonne National Laboratory

October 29, 2021 


\section{Acknowledgements}

This report was produced under the auspices of the US DOE Fuel Cycle R\&D program Advanced Reactors Regulatory Development Campaign. Issuance of this report meets milestone M3RD-21AN06010510.

This work was conducted at Argonne National Laboratory and supported by the U.S. Department of Energy, Office of Nuclear Energy, under Contract DE-AC02-06CH11357. 


\section{Table of Contents}

Abstract

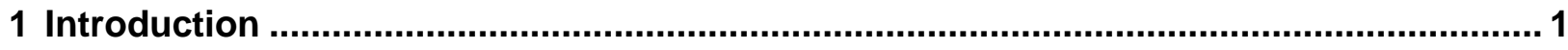

2 Molten salt spreading and heat transfer ..................................................................... 1

2.1 Visible video from tests of molten salt spreading and freezing on a stainless steel

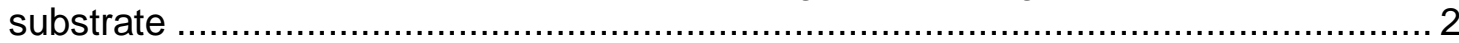

2.2 Temperature of substrate underside measured by thermocouple …….................... 2

2.3 Temperature measurements of salt and substrate surface by IR camera ................ 16

2.4 Leading edge and covered area as a function of time ........................................ 17

2.5 Thermocouple measurements of atmosphere temperature above pool ....................22

2.6 Thickness measurements of frozen salt by location ..............................................2. 24

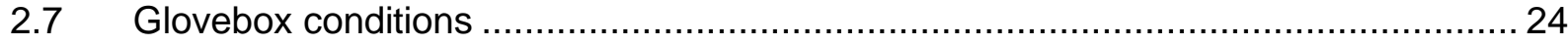

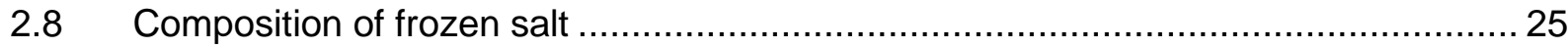

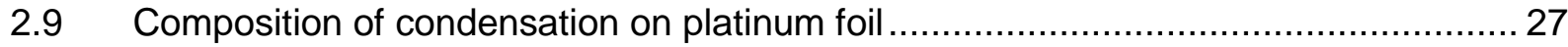

3 Molten salt flowing and freezing behavior through tubing ..........................................29

3.1 Visible video from tests of molten salt flowing on stainless steel funnel cone ............ 29

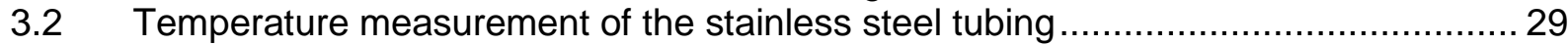

3.3 Temperature of the underside of the stainless steel beaker ….............................. 30

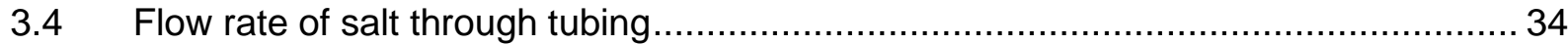

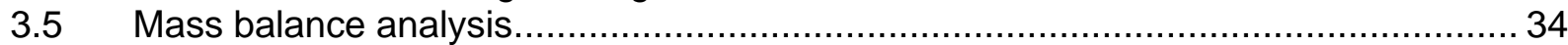

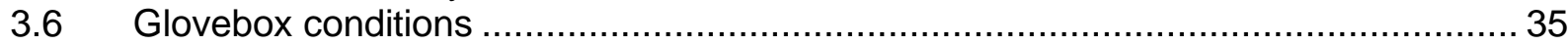

4 Stainless steel corrosion kinetics in molten salt ........................................................... 35

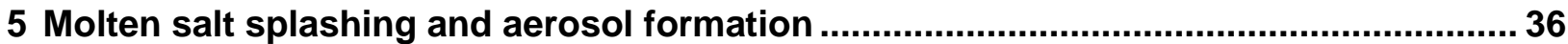

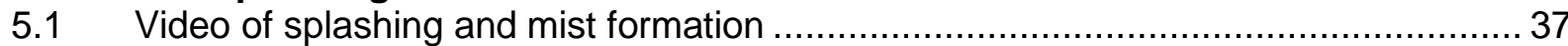

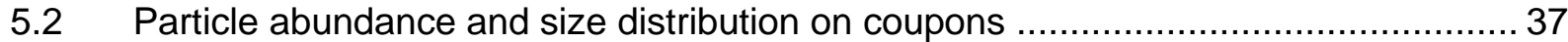

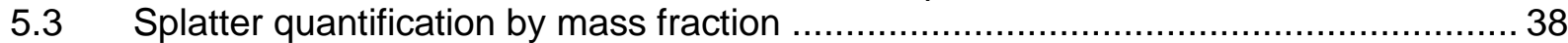

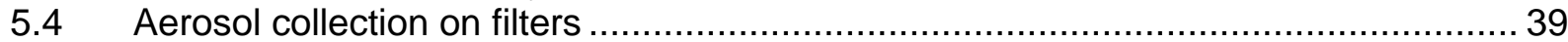

5.5 Temperatures measured in the spill containment box ...................................... 40

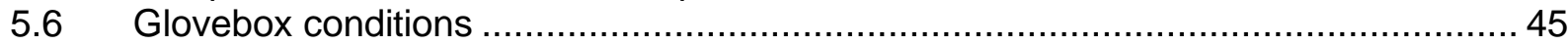

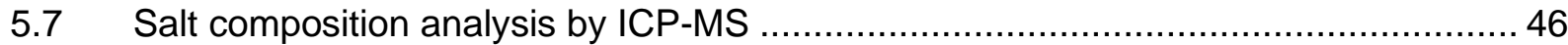

5.8 Composition of condensation on ceiling of spill containment box .......................... 47

5.9 Splatter and aerosol particle composition by SEM-EDS ..................................... 48

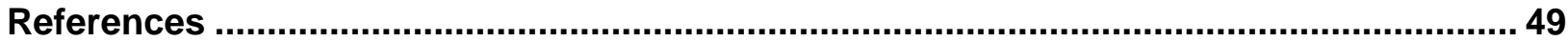




\section{Figures}

1. Location of thermocouples attached to the underside of the substrate overlaid on an image of the salt spreading path for Tests (A) 1, (B) 3, (C) 4, (D) 6, (E) 8, and (F) 9.......... 3

2. Schematic of the coordinates of the stainless steel substrate boundary relative to the direction of the salt flow.

3. Image of stainless steel sheet that shows the position of the platinum foil for condensation collection.

4. The coupon labels and locations in the catch pan relative to the salt spill zone.

5. The locations of thermocouples TC2, TC3, and TC4 that measured the temperature of the spill containment box atmosphere. 


\section{Tables}

1. Summary of spreading and heat transfer test conditions ............................................... 1

2. List of available visible video of spreading tests ............................................................ 2

3. Temp. $\left({ }^{\circ} \mathrm{C}\right)$ measured by thermocouples attached to substrate underside for Test $1 \ldots \ldots \ldots \ldots . . .4$

4. Temp. $\left({ }^{\circ} \mathrm{C}\right)$ measured by thermocouples attached to substrate underside for Test $3 \ldots \ldots \ldots \ldots . .6$

5. Temp. $\left({ }^{\circ} \mathrm{C}\right)$ measured by thermocouples attached to substrate underside for Test $4 \ldots \ldots \ldots \ldots . . .8$

6. Temp. $\left({ }^{\circ} \mathrm{C}\right)$ measured by thermocouples attached to substrate underside for Test $6 \ldots \ldots \ldots . . .10$

7. Temp. $\left({ }^{\circ} \mathrm{C}\right)$ measured by thermocouples attached to substrate underside for Test $8 \ldots \ldots \ldots \ldots . .12$

8. Temp. $\left({ }^{\circ} \mathrm{C}\right)$ measured by thermocouples attached to substrate underside for Test $9 \ldots \ldots \ldots . .14$

9. The coordinates of the corners of the stainless steel substrate in pixels .............................16

10. Leading edge position $(\mathrm{mm})$ of molten salt spreading on stainless steel substrate ..............18

11. Covered area $\left(\mathrm{mm}^{2}\right)$ of molten salt spreading on stainless steel substrate .......................20

12. Description of thermocouple location above molten salt pool ............................................22

13. Temp. $\left({ }^{\circ} \mathrm{C}\right)$ measured by thermocouple above molten salt pool for spreading tests ............22

14. Thickness of frozen salt crust and pool after spreading $(\mathrm{mm}) \ldots \ldots \ldots \ldots \ldots \ldots \ldots \ldots \ldots \ldots \ldots \ldots \ldots \ldots \ldots . .24$

15. The $\mathrm{O}_{2}$ content, $\mathrm{H}_{2} \mathrm{O}$ content, and temperature of the glovebox atmosphere measured

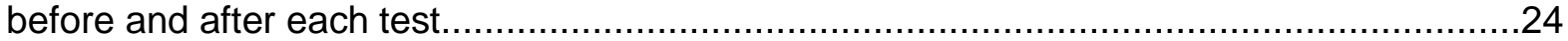

16. Concentrations of major components in FLiNaK before the tests (mol \%) …......................25

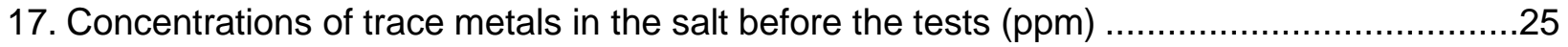

18. Composition of frozen salt samples collected from spreading tests .................................26

19. Trace metal composition of frozen salt samples collected from spreading tests .................27

20. Composition of condensate collected on platinum foil above salt pool in Test 4 .................28

21. Summary of test conditions to study molten FLiNaK flowing through tubing .......................29

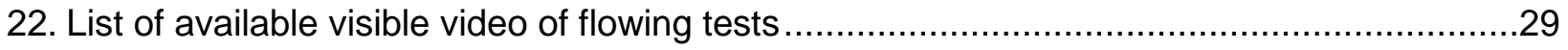

23. Information on exported IR video frames from flowing tests ...........................................30

24. Temp. $\left({ }^{\circ} \mathrm{C}\right)$ measured by thermocouple attached to underside of stainless steel beaker .....30 
25. The maximum temperature recorded at the beaker underside, the thickness of the frozen salt pool, and salt mass in beaker ...

26. The mass $(\mathrm{g})$ of salt recorded in stainless steel beaker as a function of time .......

27. Results from mass balance analysis of frozen FLiNaK salt.

28. The $\mathrm{O}_{2}$ content, $\mathrm{H}_{2} \mathrm{O}$ content, and temperature of the glovebox atmosphere measured before and after each test.

29. Summary of test conditions to study the corrosion of 316 stainless steel in molten FLiNaK

30. Summary of steady corrosion currents measured during PS tests and calculated mass corrosion rates and penetration rates....

31. Summary of splashing and aerosol generation test conditions for tests using molten FLiNaK with surrogate fission products

32. List of available visible video of molten salt splashing tests

33. Summary of total area $\left(\mathrm{mm}^{2}\right)$ analyzed per coupon for each test

34. Mass balance results of salt recovered from catch pan after freezing.....

35. Elemental composition of particulates collected on $0.45-\mu \mathrm{m}$ filters measured in water leachate

36. Elemental composition of particulates collected on $0.45-\mu \mathrm{m}$ filters after acid leach

37. Temp. $\left({ }^{\circ} \mathrm{C}\right)$ measured by thermocouple attached to underside of stainless steel catch pan

38. The temp. $\left({ }^{\circ} \mathrm{C}\right)$ of the spill containment box atmosphere by thermocouple for Test $1 \ldots \ldots \ldots . . .42$

39. The temp. $\left({ }^{\circ} \mathrm{C}\right)$ of the spill containment box atmosphere by thermocouple for Test $2 \ldots \ldots \ldots . . .42$

40. The temp. $\left({ }^{\circ} \mathrm{C}\right)$ of the spill containment box atmosphere by thermocouple for Test $3 \ldots \ldots \ldots . . .43$

41. The temp. $\left({ }^{\circ} \mathrm{C}\right)$ of the spill containment box atmosphere by thermocouple for Test $4 \ldots \ldots \ldots . . .43$

42. The temp. $\left({ }^{\circ} \mathrm{C}\right)$ of the spill containment box atmosphere by thermocouple for Test $5 \ldots \ldots \ldots . . .44$

43. The temp. $\left({ }^{\circ} \mathrm{C}\right)$ of the spill containment box atmosphere by thermocouple for Test $6 \ldots \ldots \ldots . . .44$

44. The temp. $\left({ }^{\circ} \mathrm{C}\right)$ of the spill containment box atmosphere by thermocouple for Test $7 \ldots \ldots \ldots . . .45$

45. The $\mathrm{O}_{2}$ content and $\mathrm{H}_{2} \mathrm{O}$ content of the glovebox atmosphere measured before and after each test

46. Composition of frozen salt samples collected from splash tests 
47. Trace metal composition of frozen salt samples collected from splash tests

48. Elemental composition of wipe sample from ceiling of spill containment box measured after first leach with water...

49. Elemental composition of wipe sample from ceiling of spill containment box measured after second leach with acid 


\section{Acronyms}

$\begin{array}{ll}\text { EDS } & \text { Energy dispersive X-ray spectroscopy } \\ \text { FLiNaK } & \text { LiF-NaF-KF eutectic (46.5-11.5-42 mol \%) } \\ \text { ICP-MS } & \text { Inductively coupled plasma-mass spectrometry } \\ \text { ICP-OES } & \text { Inductively coupled plasma-optical emission spectroscopy } \\ \text { IR } & \text { Infrared } \\ \text { MSR } & \text { Molten salt reactor } \\ \text { PD } & \text { Potentiodynamic } \\ \text { PS } & \text { Potentiostatic } \\ \text { PTFE } & \text { Poly(tetrafluoroethylene) } \\ \text { SEM } & \text { Scanning electron microscopy }\end{array}$




\begin{abstract}
This document provides or identifies the full suite of data that was measured in laboratory salt spill tests on molten salt spreading and heat transfer, molten salt flowing and freezing in tubing, stainless steel corrosion kinetics in molten salt, and molten salt splashing and aerosol generation. The background, motivation, and methods for the work are explained in detail in an accompanying report (Thomas and Jackson, 2021), which includes representative results for all measurements. Quantitative data are tabulated to facilitate use in follow-on calculations and qualitative data in the form of visual and infrared video files are indexed for reference.
\end{abstract}




\section{Introduction}

This document provides the full suite of data that were measured in laboratory tests to provide experimental data that can be used to parameterize and validate individual process models that predict the consequences of molten salt spill accidents. All tests were conducted with pure eutectic FLiNaK (46.5-11.5-42 mol \% LiF-NaF-KF) or eutectic FLiNaK doped with 0.9 mol \% CsF and $0.099 \mathrm{~mol} \% \mathrm{Csl}$ as surrogate fission products. The motivation for the work, the experimental approach, the experimental designs, and the methods are described in detail elsewhere (Thomas and Jackson, 2021). The results provided in this document are grouped in sections that focus on four aspects of a molten salt spill accident:

- Molten salt spreading and heat transfer on stainless steel sheets,

- Molten salt flowing and freezing in stainless steel tubing,

- Stainless steel corrosion kinetics in molten salt, and

- Molten salt splashing and aerosol generation.

The corresponding sections addressing these aspects of a molten salt spill accident in Thomas and Jackson, 2021 are identifed for convenience. Representative results are provided in Thomas and Jackson, 2021 and most of the acquired data are provided in this document. Datasets that are too large for inclusion in this document and available videos are identified in the following sections and can be provided by the authors upon reasonable request.

\section{Molten salt spreading and heat transfer}

The data provided in this section correspond to Section 3 in Thomas and Jackson, 2021, and the experimental design and methods are described therein. Table 1 provides a summary of the conditions used in each FLiNaK spreading and heat transfer test that was conducted.

Table 1: Summary of spreading and heat transfer test conditions

\begin{tabular}{ccccccc}
\hline Test & $\begin{array}{c}\text { Target initial salt } \\
\text { temp. }\left({ }^{\circ} \mathrm{C}\right)^{\mathrm{a}}\end{array}$ & $\begin{array}{c}\text { Max temp. } \\
\left({ }^{\circ} \mathrm{C}\right)^{\mathrm{b}}\end{array}$ & $\begin{array}{c}\text { Mass of salt } \\
\text { poured }(\mathrm{g})\end{array}$ & $\begin{array}{c}\text { Average pour } \\
\text { rate }\left(\mathrm{g} \mathrm{s}^{-1}\right)\end{array}$ & $\begin{array}{c}\text { Sheet tilt } \\
\text { angle }\left({ }^{\circ}\right)\end{array}$ & $\begin{array}{c}\text { CsF \& Csl } \\
\text { in salt? }\end{array}$ \\
\hline 1 & 500 & 476.7 & 28.4 & 20.8 & 2.5 & No \\
2 & 500 & 494.5 & 38.4 & 21.8 & 5.0 & No \\
3 & 550 & 530.0 & 49.0 & 18.6 & 2.5 & No \\
4 & 650 & 625.3 & 30.0 & 19.6 & 2.5 & Yes $^{\text {c }}$ \\
5 & 650 & 640.0 & 24.3 & 13.3 & 5.0 & No \\
6 & 650 & 649.0 & 29.6 & 22.2 & 2.5 & No \\
7 & 800 & 731.5 & 19.1 & 9.7 & 5.0 & No \\
8 & 800 & 734.1 & 30.3 & 13.6 & 2.5 & No \\
9 & 800 & 794.5 & 30.6 & 29.6 & 2.5 & No \\
\hline
\end{tabular}

a The actual salt temperature may have differed from the target temperature.

b The maximum temperature of the salt measured by the infrared camera (uncorrected for emissivity) provides an estimate of the actual salt temperature as it was poured.

${ }^{\mathrm{c}}$ Added $0.200 \mathrm{~g} \mathrm{Csl}$ and $0.967 \mathrm{~g} \mathrm{CsF}$ to FLiNaK with a total mass of $30.463 \mathrm{~g}$. 


\subsection{Visible video from tests of molten salt spreading and freezing on a stainless steel substrate}

Video of the molten salt spreading and freezing on the stainless steel substrate was filmed using a visible camera mounted on a tripod and is available upon reasonable request. A list of available videos is provided in Table 2.

Table 2: List of available visible video of spreading tests

\begin{tabular}{ccc}
\hline Test & Filename & Notes \\
\hline 1 & SpreadingVideo_Test1.mp4 & Video is blurry \\
2 & SpreadingVideo_Test2.mp4 & - \\
3 & SpreadingVideo_Test3.mp4 & - \\
4 & SpreadingVideo_Test4.mp4 & - \\
5 & SpreadingVideo_Test5.mp4 & - \\
6 & SpreadingVideo_Test6.mp4 & - \\
7 & SpreadingVideo_Test7.mp4 & Video taken using camera outside of glovebox \\
8 & SpreadingVideo_Test8.mp4 & - \\
9 & SpreadingVideo_Test9.mp4 & - \\
\hline
\end{tabular}

\subsection{Temperature of substrate underside measured by thermocouple}

The temperatures at the underside of the substrate surface were measured by using butt-welded thermocouple lead wires with the junction attached to the surface with thermally conductive cement. A description of the method and the performance of the method is provided in Appendix B of Thomas and Jackson, 2021.

Figure 1 shows the layout of the thermocouples attached to the underside of the stainless steel substrate overlaid on a still frame of video collected by using the infrared (IR) camera for Tests 1 , $3,4,6,8$, and 9 . These images show the positions of the salt after spreading ceased relative to the locations of the thermocouples. The pooled salt at the end of the substrate for Test 4 is blocked from the field of view of the IR camera by a platinum foil (Figure $3 \mathrm{C}$ ). The thermocouple measurements failed for Tests 2, 5, and 7 and the temperature of the underside of the substrate is unknown for these tests. The position of Thermocouple 8 (TC8) was approximately $0.5 \mathrm{in}$. from the bottom of the sheet for all tests.

The temperatures recorded by each thermocouple for Tests $1,3,4,6,8$, and 9 are provided in Table 3, Table 4, Table 5, Table 6, Table 7, and Table 8, respectively. 
(A)

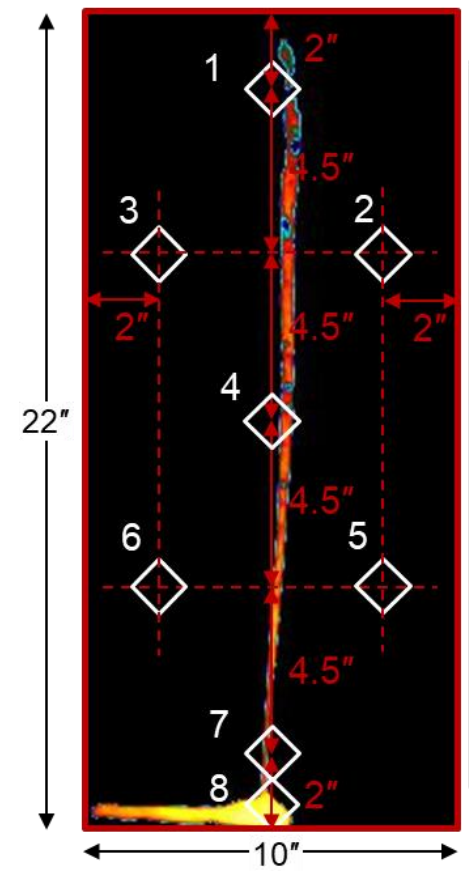

(D)

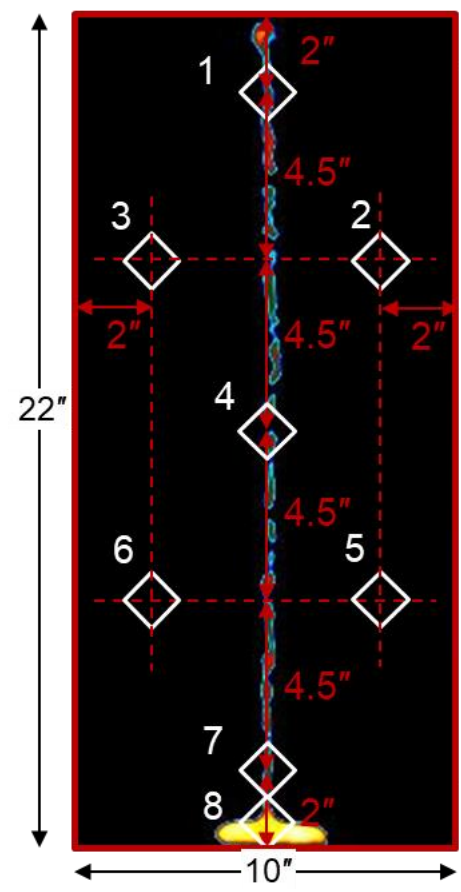

(B)

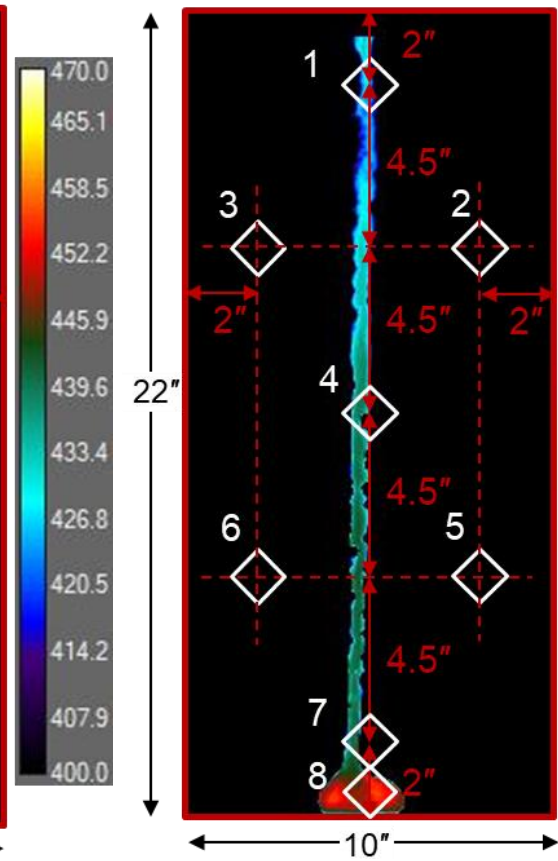

(E)

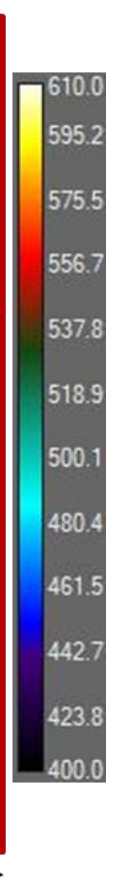

(C)

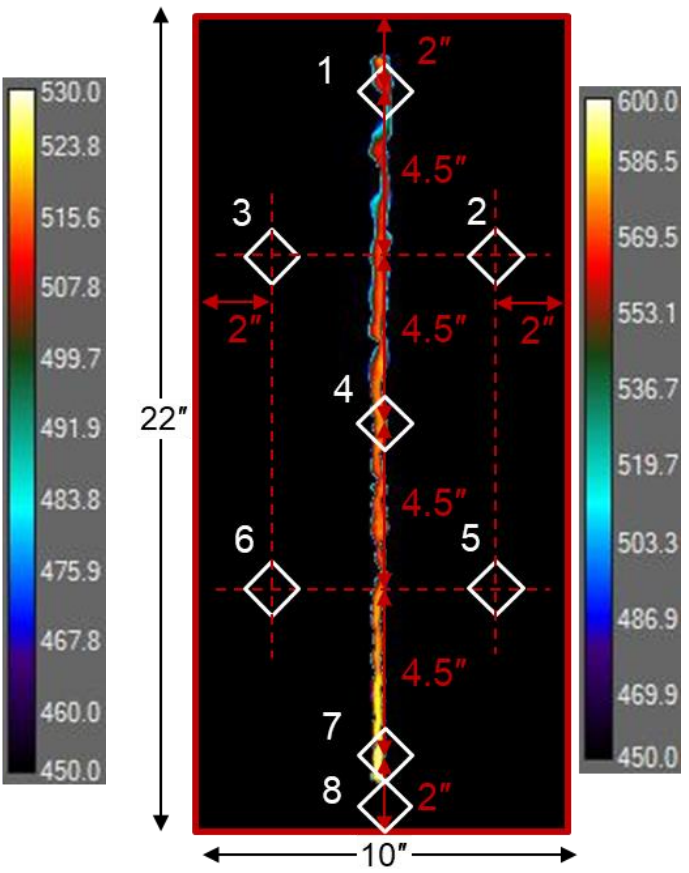

(F)

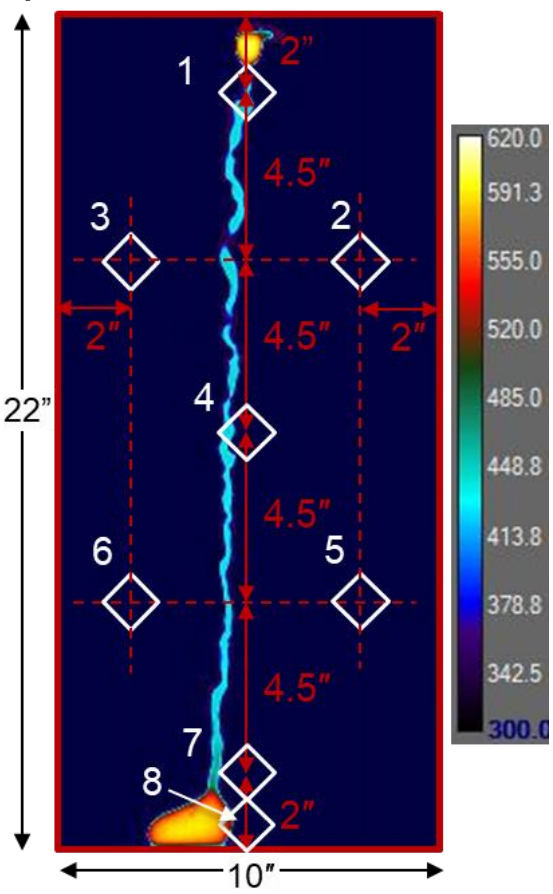

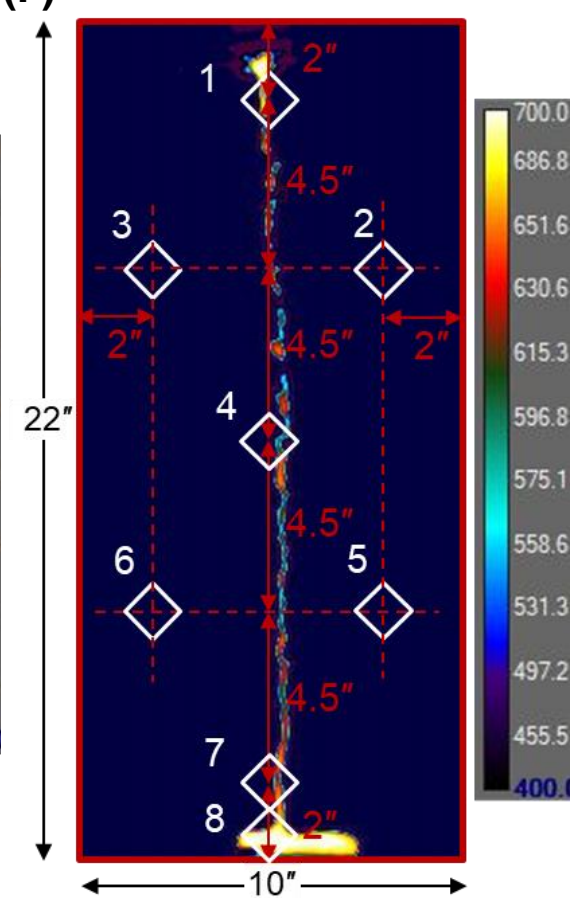

Figure 1: Location of thermocouples attached to the underside of the substrate overlaid on an image of the salt spreading path for Tests (A) 1, (B) 3, (C) 4, (D) 6, (E) 8, and (F) 9. Thermocouple 8 is positioned approximately 0.5 in. from the substrate bottom. A platinum foil blocked the pooled salt above Thermocouple 8 from the IR camera field of view for Test 4 in subplot C. 
Table 3: Temp. $\left({ }^{\circ} \mathrm{C}\right)$ measured by thermocouples attached to substrate underside for Test 1

\begin{tabular}{|c|c|c|c|c|c|c|c|c|}
\hline Time (min.) & TC1 & TC2 & TC3 & TC4 & TC5 & TC6 & TC7 & TC8 \\
\hline 0.00 & 27.0 & 26.9 & 27.8 & 28.7 & 26.7 & 27.2 & 25.3 & 26.4 \\
\hline 0.25 & 55.2 & 26.9 & 27.8 & 48.8 & 26.7 & 27.2 & 44.2 & 125.3 \\
\hline 0.50 & 58.3 & 27.0 & 27.8 & 57.7 & 26.7 & 27.2 & 60.5 & 154.3 \\
\hline 0.75 & 60.5 & 27.0 & 27.9 & 60.3 & 26.8 & 27.2 & 70.9 & 153.9 \\
\hline 1.00 & 61.0 & 27.0 & 27.9 & 61.0 & 26.8 & 27.3 & 76.1 & 149.3 \\
\hline 1.25 & 60.5 & 27.1 & 28.1 & 61.0 & 26.9 & 27.3 & 78.5 & 145.0 \\
\hline 1.50 & 59.3 & 27.1 & 28.2 & 60.7 & 27.0 & 27.4 & 79.5 & 140.9 \\
\hline 1.75 & 59.7 & 27.3 & 28.4 & 60.0 & 27.2 & 27.5 & 79.7 & 137.0 \\
\hline 2.00 & 60.3 & 27.4 & 28.7 & 59.3 & 27.4 & 27.7 & 79.6 & 133.2 \\
\hline 2.25 & 59.6 & 27.7 & 29.0 & 58.5 & 27.7 & 28.0 & 79.1 & 129.4 \\
\hline 2.50 & 58.4 & 27.9 & 29.4 & 57.7 & 28.0 & 28.2 & 78.4 & 125.6 \\
\hline 2.75 & 57.1 & 28.2 & 29.8 & 56.9 & 28.4 & 28.5 & 77.6 & 121.9 \\
\hline 3.00 & 55.7 & 28.5 & 30.3 & 56.1 & 28.7 & 28.8 & 76.7 & 118.1 \\
\hline 3.25 & 54.3 & 28.8 & 30.7 & 55.2 & 29.1 & 29.2 & 75.8 & 114.6 \\
\hline 3.50 & 52.8 & 29.1 & 31.1 & 54.3 & 29.4 & 29.5 & 74.7 & 111.4 \\
\hline 3.75 & 51.4 & 29.4 & 31.5 & 53.5 & 29.7 & 29.8 & 73.7 & 108.1 \\
\hline 4.00 & 50.1 & 29.7 & 31.9 & 52.6 & 30.1 & 30.1 & 72.6 & 104.9 \\
\hline 4.25 & 48.8 & 30.0 & 32.3 & 51.8 & 30.4 & 30.4 & 71.5 & 101.9 \\
\hline 4.50 & 47.7 & 30.3 & 32.7 & 51.0 & 30.6 & 30.6 & 70.4 & 98.9 \\
\hline 4.75 & 46.6 & 30.6 & 33.0 & 50.2 & 30.9 & 30.9 & 69.3 & 96.0 \\
\hline 5.00 & 45.6 & 30.8 & 33.3 & 49.5 & 31.1 & 31.1 & 68.2 & 93.2 \\
\hline 5.25 & 44.7 & 31.0 & 33.5 & 48.8 & 31.4 & 31.3 & 67.1 & 90.5 \\
\hline 5.50 & 43.8 & 31.1 & 33.7 & 48.1 & 31.5 & 31.5 & 66.0 & 88.0 \\
\hline 5.75 & 43.0 & 31.3 & 33.9 & 47.4 & 31.7 & 31.7 & 64.9 & 85.6 \\
\hline 6.00 & 42.2 & 31.5 & 34.1 & 46.8 & 31.9 & 31.8 & 63.8 & 83.3 \\
\hline 6.25 & 41.5 & 31.6 & 34.3 & 46.2 & 32.1 & 31.9 & 62.7 & 81.0 \\
\hline 6.50 & 40.9 & 31.7 & 34.4 & 45.6 & 32.2 & 32.0 & 61.6 & 78.9 \\
\hline 6.75 & 40.3 & 31.8 & 34.5 & 45.1 & 32.3 & 32.0 & 60.6 & 76.9 \\
\hline 7.00 & 39.7 & 31.8 & 34.6 & 44.6 & 32.5 & 32.1 & 59.6 & 75.0 \\
\hline 7.25 & 39.2 & 31.9 & 34.6 & 44.1 & 32.5 & 32.1 & 58.5 & 73.1 \\
\hline 7.50 & 38.7 & 31.9 & 34.7 & 43.6 & 32.6 & 32.2 & 57.6 & 71.3 \\
\hline 7.75 & 38.2 & 32.0 & 34.7 & 43.1 & 32.7 & 32.2 & 56.6 & 69.6 \\
\hline 8.00 & 37.7 & 32.0 & 34.7 & 42.7 & 32.8 & 32.2 & 55.7 & 67.9 \\
\hline 8.25 & 37.3 & 32.0 & 34.7 & 42.2 & 32.8 & 32.2 & 54.8 & 66.4 \\
\hline 8.50 & 36.9 & 32.0 & 34.7 & 41.8 & 32.9 & 32.2 & 53.9 & 64.9 \\
\hline 8.75 & 36.5 & 32.0 & 34.7 & 41.4 & 32.9 & 32.2 & 53.1 & 63.5 \\
\hline 9.00 & 36.2 & 31.9 & 34.7 & 41.0 & 33.0 & 32.2 & 52.3 & 62.1 \\
\hline 9.25 & 35.8 & 31.9 & 34.6 & 40.7 & 33.0 & 32.2 & 51.5 & 60.8 \\
\hline 9.50 & 35.5 & 31.9 & 34.6 & 40.4 & 33.0 & 32.2 & 50.6 & 59.6 \\
\hline 9.75 & 35.2 & 31.9 & 34.6 & 40.0 & 33.0 & 32.1 & 49.9 & 58.2 \\
\hline 10.00 & 34.9 & 31.8 & 34.5 & 39.7 & 33.0 & 32.1 & 49.1 & 56.9 \\
\hline
\end{tabular}


Table 3: (cont.)

\begin{tabular}{|c|c|c|c|c|c|c|c|c|}
\hline Time (min.) & $\mathrm{TC} 1$ & TC2 & TC3 & TC4 & TC5 & TC6 & TC7 & TC8 \\
\hline 10.25 & 34.7 & 31.8 & 34.4 & 39.4 & 33.0 & 32.0 & 48.4 & 55.7 \\
\hline 10.50 & 34.4 & 31.8 & 34.4 & 39.1 & 33.0 & 32.0 & 47.7 & 54.5 \\
\hline 10.75 & 34.2 & 31.7 & 34.3 & 38.8 & 33.0 & 32.0 & 47.0 & 53.4 \\
\hline 11.00 & 33.9 & 31.7 & 34.2 & 38.6 & 33.0 & 31.9 & 46.4 & 52.4 \\
\hline 11.25 & 33.7 & 31.6 & 34.2 & 38.3 & 33.0 & 31.9 & 45.8 & 51.5 \\
\hline 11.50 & 33.5 & 31.5 & 34.1 & 38.1 & 33.0 & 31.8 & 45.1 & 50.6 \\
\hline 11.75 & 33.2 & 31.5 & 34.0 & 37.8 & 32.9 & 31.8 & 44.5 & 49.7 \\
\hline 12.00 & 33.0 & 31.4 & 33.9 & 37.6 & 32.9 & 31.8 & 43.9 & 48.9 \\
\hline 12.25 & 32.9 & 31.4 & 33.9 & 37.4 & 32.9 & 31.7 & 43.4 & 48.1 \\
\hline 12.50 & 32.7 & 31.3 & 33.8 & 37.1 & 32.8 & 31.7 & 42.8 & 47.3 \\
\hline 12.75 & 32.5 & 31.3 & 33.7 & 36.9 & 32.8 & 31.6 & 42.3 & 46.6 \\
\hline 13.00 & 32.3 & 31.2 & 33.6 & 36.7 & 32.8 & 31.5 & 41.8 & 45.8 \\
\hline 13.25 & 32.2 & 31.2 & 33.5 & 36.5 & 32.7 & 31.5 & 41.3 & 45.2 \\
\hline 13.50 & 32.0 & 31.1 & 33.4 & 36.3 & 32.7 & 31.5 & 40.8 & 44.5 \\
\hline 13.75 & 31.9 & 31.0 & 33.4 & 36.2 & 32.6 & 31.4 & 40.4 & 43.9 \\
\hline 14.00 & 31.7 & 31.0 & 33.3 & 36.0 & 32.6 & 31.4 & 39.9 & 43.3 \\
\hline 14.25 & 31.6 & 30.9 & 33.2 & 35.8 & 32.5 & 31.3 & 39.5 & 42.8 \\
\hline 14.50 & 31.5 & 30.9 & 33.1 & 35.7 & 32.5 & 31.3 & 39.1 & 42.2 \\
\hline 14.75 & 31.4 & 30.8 & 33.0 & 35.5 & 32.4 & 31.2 & 38.7 & 41.7 \\
\hline 15.00 & 31.3 & 30.8 & 32.9 & 35.4 & 32.4 & 31.2 & 38.3 & 41.2 \\
\hline 15.25 & 31.1 & 30.7 & 32.8 & 35.2 & 32.3 & 31.1 & 37.9 & 40.8 \\
\hline 15.50 & 31.0 & 30.7 & 32.8 & 35.1 & 32.2 & 31.1 & 37.6 & 40.3 \\
\hline 15.75 & 30.9 & 30.6 & 32.7 & 34.9 & 32.2 & 31.0 & 37.2 & 39.9 \\
\hline 16.00 & 30.8 & 30.6 & 32.6 & 34.8 & 32.1 & 31.0 & 36.9 & 39.5 \\
\hline 16.25 & 30.7 & 30.5 & 32.5 & 34.6 & 32.0 & 30.9 & 36.6 & 39.1 \\
\hline 16.50 & 30.6 & 30.5 & 32.4 & 34.5 & 32.0 & 30.9 & 36.2 & 38.7 \\
\hline 16.75 & 30.6 & 30.4 & 32.4 & 34.4 & 31.9 & 30.8 & 35.9 & 38.3 \\
\hline 17.00 & 30.5 & 30.4 & 32.3 & 34.3 & 31.9 & 30.8 & 35.6 & 37.9 \\
\hline 17.25 & 30.4 & 30.3 & 32.2 & 34.2 & 31.8 & 30.7 & 35.4 & 37.6 \\
\hline 17.50 & 30.3 & 30.3 & 32.2 & 34.0 & 31.7 & 30.7 & 35.1 & 37.3 \\
\hline 17.75 & 30.2 & 30.3 & 32.0 & 33.9 & 31.7 & 30.6 & 34.8 & 37.0 \\
\hline 18.00 & 30.2 & 30.2 & 32.0 & 33.8 & 31.6 & 30.6 & 34.6 & 36.7 \\
\hline 18.25 & 30.1 & 30.2 & 31.9 & 33.7 & 31.6 & 30.5 & 34.3 & 36.4 \\
\hline 18.50 & 30.0 & 30.1 & 31.8 & 33.6 & 31.5 & 30.5 & 34.1 & 36.1 \\
\hline 18.75 & 30.0 & 30.1 & 31.8 & 33.5 & 31.4 & 30.4 & 33.9 & 35.8 \\
\hline 19.00 & 29.9 & 30.0 & 31.7 & 33.4 & 31.4 & 30.4 & 33.6 & 35.5 \\
\hline 19.25 & 29.8 & 30.0 & 31.6 & 33.3 & 31.3 & 30.4 & 33.4 & 35.3 \\
\hline 19.50 & 29.8 & 29.9 & 31.6 & 33.2 & 31.2 & 30.3 & 33.2 & 35.0 \\
\hline 19.75 & 29.7 & 29.9 & 31.5 & 33.1 & 31.2 & 30.3 & 33.0 & 34.8 \\
\hline 20.00 & 29.6 & 29.8 & 31.5 & 33.0 & 31.1 & 30.2 & 32.8 & 34.6 \\
\hline
\end{tabular}


Table 4: Temp. $\left({ }^{\circ} \mathrm{C}\right)$ measured by thermocouples attached to substrate underside for Test 3

\begin{tabular}{|c|c|c|c|c|c|c|c|c|}
\hline Time (min.) & TC1 & $\mathrm{TC2^{ \textrm {a } }}$ & TC3 & TC4 ${ }^{\mathrm{a}}$ & TC5 & TC6 & TC7 & TC8 \\
\hline 0.00 & 25.9 & - & 26.8 & - & 25.4 & 26.3 & 24.0 & 25.2 \\
\hline 0.25 & 100.8 & - & 26.8 & - & 25.4 & 26.3 & 24.1 & 44.9 \\
\hline 0.50 & 115.8 & - & 26.9 & - & 25.5 & 26.3 & 41.8 & 133.4 \\
\hline 0.75 & 114.2 & - & 26.9 & - & 25.5 & 26.3 & 78.2 & 198.2 \\
\hline 1.00 & 109.3 & - & 27.1 & - & 25.5 & 26.5 & 111.4 & 230.3 \\
\hline 1.25 & 103.9 & - & 27.5 & - & 25.6 & 27.0 & 127.8 & 229.2 \\
\hline 1.50 & 103.2 & - & 28.2 & - & 25.7 & 27.7 & 139.1 & 216.9 \\
\hline 1.75 & 102.1 & - & 29.1 & - & 25.8 & 28.5 & 147.5 & 206.0 \\
\hline 2.00 & 97.7 & - & 30.1 & - & 25.9 & 29.5 & 152.6 & 199.0 \\
\hline 2.25 & 92.5 & - & 31.2 & - & 26.2 & 30.5 & 155.3 & 193.6 \\
\hline 2.50 & 87.7 & - & 32.3 & - & 26.5 & 31.5 & 156.0 & 188.9 \\
\hline 2.75 & 83.1 & - & 33.3 & - & 26.8 & 32.4 & 155.3 & 184.3 \\
\hline 3.00 & 79.1 & - & 34.3 & - & 27.2 & 33.2 & 153.8 & 180.0 \\
\hline 3.25 & 75.4 & - & 35.2 & - & 27.6 & 33.9 & 151.7 & 175.8 \\
\hline 3.50 & 72.1 & - & 35.9 & - & 28.0 & 34.5 & 149.2 & 171.3 \\
\hline 3.75 & 69.1 & - & 36.6 & - & 28.4 & 35.1 & 146.4 & 166.6 \\
\hline 4.00 & 66.4 & - & 37.1 & - & 28.8 & 35.5 & 143.3 & 161.9 \\
\hline 4.25 & 64.0 & - & 37.6 & - & 29.2 & 35.9 & 140.2 & 157.1 \\
\hline 4.50 & 61.8 & - & 38.0 & - & 29.6 & 36.3 & 137.1 & 152.5 \\
\hline 4.75 & 59.9 & - & 38.3 & - & 29.9 & 36.6 & 133.9 & 147.9 \\
\hline 5.00 & 58.0 & - & 38.5 & - & 30.2 & 36.8 & 130.7 & 143.3 \\
\hline 5.25 & 56.3 & - & 38.7 & - & 30.5 & 37.0 & 127.6 & 138.9 \\
\hline 5.50 & 54.8 & - & 38.8 & - & 30.8 & 37.2 & 124.5 & 134.6 \\
\hline 5.75 & 53.3 & - & 38.9 & - & 31.0 & 37.3 & 121.4 & 130.6 \\
\hline 6.00 & 52.0 & - & 39.0 & - & 31.3 & 37.4 & 118.4 & 126.7 \\
\hline 6.25 & 50.8 & - & 39.0 & - & 31.5 & 37.5 & 115.5 & 123.7 \\
\hline 6.50 & 49.6 & - & 39.0 & - & 31.7 & 37.6 & 113.4 & 123.8 \\
\hline 6.75 & 48.5 & - & 38.9 & - & 31.9 & 37.6 & 111.8 & 123.5 \\
\hline 7.00 & 47.5 & - & 38.8 & - & 32.0 & 37.6 & 110.3 & 122.3 \\
\hline 7.25 & 46.6 & - & 38.7 & - & 32.2 & 37.6 & 108.7 & 120.3 \\
\hline 7.50 & 45.8 & - & 38.6 & - & 32.3 & 37.6 & 106.9 & 117.9 \\
\hline 7.75 & 44.9 & - & 38.5 & - & 32.4 & 37.7 & 105.0 & 115.3 \\
\hline 8.00 & 44.2 & - & 38.4 & - & 32.5 & 37.6 & 103.0 & 112.6 \\
\hline 8.25 & 43.5 & - & 38.2 & - & 32.6 & 37.6 & 100.9 & 109.9 \\
\hline 8.50 & 42.8 & - & 38.1 & - & 32.7 & 37.6 & 98.8 & 107.2 \\
\hline 8.75 & 42.1 & - & 37.9 & - & 32.8 & 37.5 & 96.7 & 104.6 \\
\hline 9.00 & 41.5 & - & 37.8 & - & 32.9 & 37.5 & 94.8 & 102.1 \\
\hline 9.25 & 41.0 & - & 37.6 & - & 32.9 & 37.4 & 92.9 & 99.6 \\
\hline 9.50 & 40.4 & - & 37.5 & - & 32.9 & 37.4 & 90.9 & 97.3 \\
\hline 9.75 & 39.9 & - & 37.3 & - & 33.0 & 37.4 & 88.9 & 95.0 \\
\hline
\end{tabular}


Table 4: (cont.)

\begin{tabular}{|c|c|c|c|c|c|c|c|c|}
\hline Time (min.) & TC1 & TC2 $2^{a}$ & TC3 & $\mathrm{TC} 4^{\mathrm{a}}$ & TC5 & TC6 & TC7 & TC8 \\
\hline 10.00 & 39.4 & - & 37.1 & - & 33.0 & 37.3 & 87.0 & 92.8 \\
\hline 10.25 & 38.9 & - & 37.0 & - & 33.0 & 37.3 & 85.1 & 90.6 \\
\hline 10.50 & 38.5 & - & 36.8 & - & 33.1 & 37.2 & 83.4 & 88.6 \\
\hline 10.75 & 38.1 & - & 36.6 & - & 33.1 & 37.2 & 81.7 & 86.6 \\
\hline 11.00 & 37.7 & - & 36.5 & - & 33.1 & 37.1 & 80.0 & 84.6 \\
\hline 11.25 & 37.3 & - & 36.3 & - & 33.1 & 37.0 & 78.4 & 82.8 \\
\hline 11.50 & 36.9 & - & 36.1 & - & 33.1 & 37.0 & 76.9 & 81.0 \\
\hline 11.75 & 36.6 & - & 36.0 & - & 33.1 & 36.9 & 75.4 & 79.3 \\
\hline 12.00 & 36.2 & - & 35.8 & - & 33.0 & 36.9 & 73.9 & 77.6 \\
\hline 12.25 & 35.9 & - & 35.7 & - & 33.0 & 36.8 & 72.5 & 76.1 \\
\hline 12.50 & 35.6 & - & 35.5 & - & 33.0 & 36.8 & 71.2 & 74.5 \\
\hline 12.75 & 35.3 & - & 35.4 & - & 33.0 & 36.7 & 69.8 & 73.1 \\
\hline 13.00 & 35.1 & - & 35.2 & - & 32.9 & 36.6 & 68.6 & 71.6 \\
\hline 13.25 & 34.8 & - & 35.1 & - & 32.9 & 36.5 & 67.4 & 70.3 \\
\hline 13.50 & 34.5 & - & 34.9 & - & 32.9 & 36.5 & 66.2 & 69.0 \\
\hline 13.75 & 34.3 & - & 34.8 & - & 32.8 & 36.4 & 65.1 & 67.7 \\
\hline 14.00 & 34.0 & _- & 34.6 & _ & 32.8 & 36.3 & 64.0 & 66.4 \\
\hline 14.25 & 33.8 & - & 34.5 & - & 32.7 & 36.2 & 62.9 & 65.2 \\
\hline 14.50 & 33.6 & - & 34.4 & - & 32.7 & 36.1 & 61.9 & 64.0 \\
\hline 14.75 & 33.4 & - & 34.2 & - & 32.6 & 36.0 & 60.9 & 62.9 \\
\hline 15.00 & 33.2 & - & 34.1 & - & 32.6 & 35.9 & 59.9 & 61.9 \\
\hline 15.25 & 32.9 & - & 33.9 & - & 32.5 & 35.8 & 59.0 & 60.9 \\
\hline 15.50 & 32.8 & - & 33.8 & - & 32.5 & 35.7 & 58.1 & 60.0 \\
\hline 15.75 & 32.6 & - & 33.7 & - & 32.4 & 35.6 & 57.2 & 59.1 \\
\hline 16.00 & 32.4 & - & 33.5 & - & 32.4 & 35.6 & 56.3 & 58.2 \\
\hline 16.25 & 32.2 & - & 33.4 & - & 32.3 & 35.5 & 55.4 & 57.3 \\
\hline 16.50 & 32.1 & - & 33.3 & - & 32.3 & 35.4 & 54.6 & 56.5 \\
\hline 16.75 & 31.9 & - & 33.2 & - & 32.2 & 35.4 & 53.8 & 55.7 \\
\hline 17.00 & 31.8 & - & 33.1 & - & 32.2 & 35.3 & 53.1 & 54.9 \\
\hline 17.25 & 31.6 & - & 33.0 & - & 32.1 & 35.2 & 52.3 & 54.1 \\
\hline 17.50 & 31.5 & - & 32.9 & - & 32.0 & 35.1 & 51.6 & 53.4 \\
\hline 17.75 & 31.3 & - & 32.8 & - & 32.0 & 35.0 & 50.9 & 52.7 \\
\hline 18.00 & 31.2 & - & 32.7 & - & 31.9 & 34.9 & 50.2 & 52.0 \\
\hline 18.25 & 31.1 & - & 32.6 & - & 31.9 & 34.8 & 49.6 & 51.3 \\
\hline 18.50 & 30.9 & - & 32.5 & - & 31.8 & 34.8 & 49.0 & 50.7 \\
\hline 18.75 & 30.8 & - & 32.4 & - & 31.7 & 34.7 & 48.4 & 50.0 \\
\hline 19.00 & 30.7 & - & 32.3 & - & 31.7 & 34.6 & 47.8 & 49.5 \\
\hline 19.25 & 30.6 & - & 32.2 & - & 31.6 & 34.5 & 47.2 & 48.9 \\
\hline 19.50 & 30.5 & - & 32.1 & - & 31.5 & 34.4 & 46.7 & 48.3 \\
\hline 19.75 & 30.4 & - & 32.0 & - & 31.5 & 34.3 & 46.1 & 47.8 \\
\hline 20.00 & 30.3 & - & 31.9 & - & 31.4 & 34.2 & 45.6 & 47.3 \\
\hline
\end{tabular}

${ }^{\mathrm{a}} \mathrm{TC} 2$ and TC4 failed during the test. 
Table 5: Temp. $\left({ }^{\circ} \mathrm{C}\right)$ measured by thermocouples attached to substrate underside for Test 4

\begin{tabular}{|c|c|c|c|c|c|c|c|c|}
\hline Time (min.) & TC1 & TC2 & TC3 & TC4 & TC5 & TC6 & TC7 & TC8 \\
\hline 0.00 & 26.2 & 26.0 & 27.0 & 27.8 & 25.6 & 26.2 & 24.0 & 25.3 \\
\hline 0.25 & 63.2 & 26.0 & 27.0 & 90.2 & 25.6 & 26.2 & 96.5 & 194.0 \\
\hline 0.50 & 88.7 & 26.0 & 27.1 & 109.4 & 25.7 & 26.3 & 127.1 & 248.3 \\
\hline 0.75 & 103.3 & 26.1 & 27.3 & 113.8 & 25.7 & 26.3 & 146.5 & 269.5 \\
\hline 1.00 & 107.8 & 26.2 & 27.4 & 112.2 & 25.9 & 26.4 & 156.2 & 265.5 \\
\hline 1.25 & 107.1 & 26.4 & 27.6 & 108.4 & 26.1 & 26.5 & 159.1 & 254.6 \\
\hline 1.50 & 104.0 & 26.7 & 27.9 & 104.4 & 26.4 & 26.7 & 158.7 & 243.7 \\
\hline 1.75 & 99.9 & 27.0 & 28.3 & 100.5 & 26.8 & 27.0 & 156.4 & 234.4 \\
\hline 2.00 & 95.4 & 27.4 & 28.8 & 96.4 & 27.3 & 27.4 & 153.1 & 226.4 \\
\hline 2.25 & 90.8 & 27.9 & 29.4 & 92.5 & 27.9 & 27.9 & 149.3 & 219.0 \\
\hline 2.50 & 86.3 & 28.4 & 30.0 & 88.7 & 28.5 & 28.4 & 145.3 & 211.6 \\
\hline 2.75 & 82.0 & 28.9 & 30.7 & 85.2 & 29.2 & 28.9 & 141.3 & 204.3 \\
\hline 3.00 & 78.0 & 29.5 & 31.4 & 82.0 & 29.8 & 29.5 & 137.4 & 197.0 \\
\hline 3.25 & 74.4 & 30.0 & 32.0 & 79.0 & 30.5 & 30.0 & 133.6 & 190.0 \\
\hline 3.50 & 71.0 & 30.5 & 32.6 & 76.3 & 31.1 & 30.5 & 130.0 & 183.2 \\
\hline 3.75 & 67.9 & 31.0 & 33.2 & 73.8 & 31.6 & 31.0 & 126.4 & 176.8 \\
\hline 4.00 & 65.1 & 31.4 & 33.8 & 71.5 & 32.1 & 31.5 & 123.0 & 170.5 \\
\hline 4.25 & 62.5 & 31.8 & 34.3 & 69.4 & 32.6 & 31.9 & 119.8 & 164.6 \\
\hline 4.50 & 60.2 & 32.2 & 34.8 & 67.5 & 33.0 & 32.3 & 116.6 & 159.1 \\
\hline 4.75 & 58.1 & 32.6 & 35.2 & 65.7 & 33.5 & 32.7 & 113.6 & 153.8 \\
\hline 5.00 & 56.1 & 32.9 & 35.6 & 64.0 & 33.8 & 33.1 & 110.8 & 148.7 \\
\hline 5.25 & 54.3 & 33.1 & 35.9 & 62.5 & 34.1 & 33.4 & 108.0 & 143.9 \\
\hline 5.50 & 52.7 & 33.4 & 36.2 & 61.1 & 34.4 & 33.7 & 105.3 & 139.3 \\
\hline 5.75 & 51.1 & 33.5 & 36.4 & 59.8 & 34.7 & 33.9 & 102.7 & 134.9 \\
\hline 6.00 & 49.7 & 33.7 & 36.6 & 58.5 & 34.9 & 34.2 & 100.2 & 130.7 \\
\hline 6.25 & 48.5 & 33.8 & 36.8 & 57.3 & 35.1 & 34.4 & 97.8 & 126.7 \\
\hline 6.50 & 47.3 & 33.9 & 36.9 & 56.2 & 35.2 & 34.5 & 95.5 & 122.9 \\
\hline 6.75 & 46.2 & 34.0 & 37.0 & 55.2 & 35.4 & 34.7 & 93.2 & 119.3 \\
\hline 7.00 & 45.2 & 34.1 & 37.1 & 54.2 & 35.5 & 34.8 & 91.1 & 115.9 \\
\hline 7.25 & 44.2 & 34.1 & 37.2 & 53.3 & 35.6 & 35.0 & 89.0 & 112.5 \\
\hline 7.50 & 43.4 & 34.1 & 37.2 & 52.4 & 35.6 & 35.1 & 86.9 & 109.3 \\
\hline 7.75 & 42.5 & 34.1 & 37.2 & 51.6 & 35.7 & 35.1 & 84.9 & 106.1 \\
\hline 8.00 & 41.7 & 34.1 & 37.2 & 50.8 & 35.7 & 35.2 & 83.0 & 103.1 \\
\hline 8.25 & 41.0 & 34.1 & 37.2 & 50.1 & 35.7 & 35.3 & 81.1 & 100.1 \\
\hline 8.50 & 40.4 & 34.1 & 37.2 & 49.3 & 35.7 & 35.3 & 79.3 & 97.4 \\
\hline 8.75 & 39.8 & 34.0 & 37.1 & 48.6 & 35.7 & 35.3 & 77.4 & 94.7 \\
\hline 9.00 & 39.3 & 34.0 & 37.0 & 47.9 & 35.7 & 35.2 & 75.8 & 92.2 \\
\hline 9.25 & 38.7 & 33.9 & 37.0 & 47.3 & 35.6 & 35.2 & 74.2 & 89.7 \\
\hline 9.50 & 38.2 & 33.8 & 36.9 & 46.8 & 35.6 & 35.2 & 72.6 & 87.4 \\
\hline 9.75 & 37.7 & 33.7 & 36.8 & 46.2 & 35.6 & 35.2 & 71.1 & 85.2 \\
\hline
\end{tabular}


Table 5: (cont.)

\begin{tabular}{|c|c|c|c|c|c|c|c|c|}
\hline Time (min.) & TC1 & TC2 & TC3 & TC4 & TC5 & TC6 & TC7 & TC8 \\
\hline 10.00 & 37.2 & 33.6 & 36.7 & 45.7 & 35.5 & 35.2 & 69.7 & 83.0 \\
\hline 10.25 & 36.8 & 33.6 & 36.6 & 45.1 & 35.5 & 35.2 & 68.3 & 81.0 \\
\hline 10.50 & 36.3 & 33.5 & 36.5 & 44.7 & 35.4 & 35.2 & 66.9 & 79.0 \\
\hline 10.75 & 35.9 & 33.4 & 36.4 & 44.2 & 35.3 & 35.1 & 65.6 & 77.1 \\
\hline 11.00 & 35.6 & 33.3 & 36.3 & 43.7 & 35.3 & 35.1 & 64.4 & 75.3 \\
\hline 11.25 & 35.2 & 33.3 & 36.1 & 43.3 & 35.2 & 35.1 & 63.2 & 73.6 \\
\hline 11.50 & 34.9 & 33.2 & 36.0 & 42.9 & 35.1 & 35.0 & 62.0 & 72.0 \\
\hline 11.75 & 34.5 & 33.1 & 35.9 & 42.5 & 35.0 & 35.0 & 60.9 & 70.4 \\
\hline 12.00 & 34.2 & 33.0 & 35.8 & 42.1 & 34.9 & 34.9 & 59.8 & 68.9 \\
\hline 12.25 & 33.9 & 32.9 & 35.7 & 41.7 & 34.8 & 34.9 & 58.8 & 67.6 \\
\hline 12.50 & 33.7 & 32.8 & 35.5 & 41.4 & 34.8 & 34.8 & 57.8 & 66.2 \\
\hline 12.75 & 33.4 & 32.7 & 35.4 & 41.0 & 34.7 & 34.7 & 56.8 & 64.9 \\
\hline 13.00 & 33.1 & 32.7 & 35.3 & 40.7 & 34.6 & 34.7 & 55.9 & 63.6 \\
\hline 13.25 & 32.9 & 32.6 & 35.1 & 40.4 & 34.5 & 34.6 & 55.0 & 62.4 \\
\hline 13.50 & 32.7 & 32.5 & 35.0 & 40.1 & 34.4 & 34.5 & 54.1 & 61.2 \\
\hline 13.75 & 32.4 & 32.4 & 34.9 & 39.8 & 34.3 & 34.5 & 53.3 & 60.1 \\
\hline 14.00 & 32.2 & 32.3 & 34.8 & 39.5 & 34.2 & 34.4 & 52.4 & 59.0 \\
\hline 14.25 & 32.0 & 32.2 & 34.6 & 39.3 & 34.2 & 34.3 & 51.6 & 58.0 \\
\hline 14.50 & 31.9 & 32.1 & 34.5 & 39.0 & 34.1 & 34.3 & 50.9 & 56.7 \\
\hline 14.75 & 31.7 & 32.0 & 34.4 & 38.7 & 34.0 & 34.2 & 50.1 & 55.3 \\
\hline 15.00 & 31.5 & 31.9 & 34.3 & 38.5 & 33.9 & 34.1 & 49.4 & 54.2 \\
\hline 15.25 & 31.3 & 31.8 & 34.1 & 38.3 & 33.8 & 34.1 & 48.6 & 53.1 \\
\hline 15.50 & 31.2 & 31.6 & 34.0 & 38.0 & 33.7 & 34.0 & 48.0 & 52.2 \\
\hline 15.75 & 31.0 & 31.5 & 33.9 & 37.8 & 33.6 & 33.9 & 47.3 & 51.4 \\
\hline 16.00 & 30.9 & 31.4 & 33.8 & 37.6 & 33.5 & 33.8 & 46.7 & 50.5 \\
\hline 16.25 & 30.8 & 31.3 & 33.7 & 37.4 & 33.4 & 33.7 & 46.0 & 49.7 \\
\hline 16.50 & 30.6 & 31.2 & 33.5 & 37.2 & 33.3 & 33.7 & 45.5 & 49.0 \\
\hline 16.75 & 30.5 & 31.2 & 33.4 & 37.0 & 33.2 & 33.6 & 44.9 & 48.3 \\
\hline 17.00 & 30.4 & 31.1 & 33.3 & 36.8 & 33.1 & 33.5 & 44.3 & 47.6 \\
\hline 17.25 & 30.3 & 31.0 & 33.2 & 36.6 & 33.0 & 33.4 & 43.8 & 47.0 \\
\hline 17.50 & 30.2 & 31.0 & 33.1 & 36.4 & 32.9 & 33.3 & 43.3 & 46.4 \\
\hline 17.75 & 30.1 & 30.9 & 33.0 & 36.3 & 32.9 & 33.2 & 42.8 & 45.8 \\
\hline 18.00 & 30.0 & 30.8 & 32.9 & 36.1 & 32.8 & 33.2 & 42.3 & 45.2 \\
\hline 18.25 & 29.9 & 30.7 & 32.8 & 36.0 & 32.7 & 33.1 & 41.8 & 44.6 \\
\hline 18.50 & 29.8 & 30.7 & 32.7 & 35.8 & 32.6 & 33.0 & 41.4 & 44.0 \\
\hline 18.75 & 29.7 & 30.6 & 32.6 & 35.6 & 32.5 & 32.9 & 40.9 & 43.5 \\
\hline 19.00 & 29.6 & 30.5 & 32.5 & 35.5 & 32.4 & 32.9 & 40.5 & 43.0 \\
\hline 19.25 & 29.5 & 30.4 & 32.4 & 35.3 & 32.3 & 32.8 & 40.1 & 42.5 \\
\hline 19.50 & 29.4 & 30.4 & 32.4 & 35.2 & 32.3 & 32.7 & 39.7 & 42.0 \\
\hline 19.75 & 29.3 & 30.3 & 32.3 & 35.0 & 32.2 & 32.6 & 39.3 & 41.6 \\
\hline 20.00 & 29.3 & 30.3 & 32.2 & 34.9 & 32.1 & 32.5 & 38.9 & 41.1 \\
\hline
\end{tabular}


Table 6: Temp. $\left({ }^{\circ} \mathrm{C}\right)$ measured by thermocouples attached to substrate underside for Test 6

\begin{tabular}{|c|c|c|c|c|c|c|c|c|}
\hline Time (min.) & TC1 & TC2 & TC3 & TC4 & TC5 & TC6 & TC7 & TC8 \\
\hline 0.00 & 27.7 & 27.6 & 28.6 & 29.6 & 27.4 & 28.0 & 25.8 & 27.3 \\
\hline 0.25 & 51.2 & 27.7 & 28.6 & 54.8 & 27.4 & 28.0 & 69.4 & 169.1 \\
\hline 0.50 & 70.1 & 27.7 & 28.7 & 71.4 & 27.4 & 28.1 & 71.5 & 233.4 \\
\hline 0.75 & 79.6 & 27.8 & 28.8 & 79.6 & 27.4 & 28.2 & 73.0 & 271.5 \\
\hline 1.00 & 84.5 & 27.8 & 29.0 & 83.6 & 27.5 & 28.3 & 77.5 & 296.8 \\
\hline 1.25 & 86.9 & 27.9 & 29.3 & 85.4 & 27.6 & 28.5 & 84.2 & 300.0 \\
\hline 1.50 & 87.6 & 28.0 & 29.7 & 85.5 & 27.7 & 28.7 & 91.0 & 292.8 \\
\hline 1.75 & 87.2 & 28.2 & 30.3 & 84.9 & 27.9 & 29.1 & 96.7 & 282.0 \\
\hline 2.00 & 85.9 & 28.3 & 31.1 & 83.7 & 28.1 & 29.6 & 101.1 & 268.9 \\
\hline 2.25 & 84.3 & 28.6 & 31.9 & 82.3 & 28.4 & 30.1 & 104.1 & 255.4 \\
\hline 2.50 & 82.3 & 28.9 & 32.7 & 80.8 & 28.8 & 30.6 & 106.0 & 242.6 \\
\hline 2.75 & 80.1 & 29.2 & 33.6 & 79.2 & 29.1 & 31.1 & 107.0 & 230.6 \\
\hline 3.00 & 77.8 & 29.6 & 34.5 & 77.5 & 29.5 & 31.6 & 107.2 & 219.7 \\
\hline 3.25 & 75.6 & 30.0 & 35.3 & 75.8 & 29.8 & 32.1 & 107.0 & 209.5 \\
\hline 3.50 & 73.4 & 30.4 & 36.1 & 74.1 & 30.2 & 32.6 & 106.3 & 200.1 \\
\hline 3.75 & 71.3 & 30.8 & 36.8 & 72.3 & 30.5 & 33.0 & 105.4 & 191.4 \\
\hline 4.00 & 69.2 & 31.2 & 37.5 & 70.7 & 30.9 & 33.5 & 104.1 & 183.3 \\
\hline 4.25 & 67.2 & 31.6 & 38.0 & 69.1 & 31.2 & 33.8 & 102.8 & 175.7 \\
\hline 4.50 & 65.4 & 31.9 & 38.6 & 67.6 & 31.5 & 34.2 & 101.2 & 168.8 \\
\hline 4.75 & 63.6 & 32.3 & 39.0 & 66.1 & 31.7 & 34.5 & 99.5 & 162.3 \\
\hline 5.00 & 61.9 & 32.6 & 39.4 & 64.7 & 32.0 & 34.7 & 97.8 & 156.2 \\
\hline 5.25 & 60.3 & 32.9 & 39.8 & 63.3 & 32.2 & 35.0 & 96.0 & 150.5 \\
\hline 5.50 & 58.7 & 33.2 & 40.1 & 62.0 & 32.4 & 35.2 & 94.3 & 145.1 \\
\hline 5.75 & 57.3 & 33.4 & 40.3 & 60.8 & 32.6 & 35.3 & 92.4 & 140.0 \\
\hline 6.00 & 55.9 & 33.7 & 40.5 & 59.6 & 32.8 & 35.5 & 90.7 & 135.2 \\
\hline 6.25 & 54.6 & 33.9 & 40.7 & 58.5 & 33.0 & 35.6 & 88.9 & 130.6 \\
\hline 6.50 & 53.3 & 34.1 & 40.8 & 57.4 & 33.1 & 35.7 & 87.2 & 126.3 \\
\hline 6.75 & 52.2 & 34.2 & 40.9 & 56.4 & 33.2 & 35.8 & 85.5 & 122.2 \\
\hline 7.00 & 51.1 & 34.4 & 40.9 & 55.4 & 33.4 & 35.9 & 83.8 & 118.3 \\
\hline 7.25 & 50.1 & 34.5 & 40.9 & 54.5 & 33.5 & 36.0 & 82.1 & 114.7 \\
\hline 7.50 & 49.1 & 34.6 & 40.9 & 53.7 & 33.6 & 36.1 & 80.5 & 111.2 \\
\hline 7.75 & 48.1 & 34.7 & 40.9 & 52.9 & 33.7 & 36.1 & 78.9 & 107.9 \\
\hline 8.00 & 47.3 & 34.8 & 40.9 & 52.1 & 33.7 & 36.2 & 77.4 & 104.8 \\
\hline 8.25 & 46.5 & 34.8 & 40.8 & 51.3 & 33.8 & 36.2 & 75.9 & 101.8 \\
\hline 8.50 & 45.7 & 34.9 & 40.7 & 50.6 & 33.9 & 36.2 & 74.5 & 99.0 \\
\hline 8.75 & 44.9 & 34.9 & 40.6 & 50.0 & 33.9 & 36.2 & 73.1 & 96.3 \\
\hline 9.00 & 44.2 & 34.9 & 40.5 & 49.3 & 34.0 & 36.2 & 71.7 & 93.7 \\
\hline 9.25 & 43.6 & 34.9 & 40.4 & 48.7 & 34.0 & 36.2 & 70.4 & 91.3 \\
\hline 9.50 & 42.9 & 34.9 & 40.3 & 48.1 & 34.1 & 36.2 & 69.1 & 88.9 \\
\hline 9.75 & 42.3 & 34.9 & 40.1 & 47.6 & 34.1 & 36.2 & 67.8 & 86.7 \\
\hline
\end{tabular}


Table 6: (cont.)

\begin{tabular}{|c|c|c|c|c|c|c|c|c|}
\hline Time (min.) & TC1 & TC2 & TC3 & TC4 & TC5 & TC6 & TC7 & TC8 \\
\hline 10.00 & 41.7 & 34.9 & 40.0 & 47.0 & 34.1 & 36.2 & 66.6 & 84.6 \\
\hline 10.25 & 41.2 & 34.9 & 39.9 & 46.5 & 34.1 & 36.2 & 65.4 & 82.5 \\
\hline 10.50 & 40.7 & 34.9 & 39.8 & 46.0 & 34.1 & 36.2 & 64.3 & 80.6 \\
\hline 10.75 & 40.2 & 34.8 & 39.6 & 45.5 & 34.1 & 36.1 & 63.2 & 78.7 \\
\hline 11.00 & 39.7 & 34.8 & 39.5 & 45.1 & 34.1 & 36.1 & 62.1 & 76.9 \\
\hline 11.25 & 39.3 & 34.7 & 39.3 & 44.6 & 34.1 & 36.0 & 61.0 & 75.2 \\
\hline 11.50 & 38.9 & 34.6 & 39.2 & 44.2 & 34.1 & 36.0 & 60.1 & 73.6 \\
\hline 11.75 & 38.4 & 34.6 & 39.0 & 43.8 & 34.0 & 36.0 & 59.1 & 72.1 \\
\hline 12.00 & 38.1 & 34.5 & 38.9 & 43.4 & 34.0 & 35.9 & 58.2 & 70.6 \\
\hline 12.25 & 37.7 & 34.4 & 38.7 & 43.0 & 33.9 & 35.9 & 57.3 & 69.2 \\
\hline 12.50 & 37.3 & 34.3 & 38.6 & 42.7 & 33.9 & 35.8 & 56.4 & 67.8 \\
\hline 12.75 & 37.0 & 34.3 & 38.4 & 42.3 & 33.9 & 35.8 & 55.5 & 66.5 \\
\hline 13.00 & 36.7 & 34.2 & 38.3 & 42.0 & 33.8 & 35.7 & 54.7 & 65.2 \\
\hline 13.25 & 36.4 & 34.1 & 38.1 & 41.7 & 33.8 & 35.7 & 53.9 & 64.0 \\
\hline 13.50 & 36.1 & 34.0 & 38.0 & 41.3 & 33.7 & 35.6 & 53.1 & 62.8 \\
\hline 13.75 & 35.8 & 33.9 & 37.9 & 41.1 & 33.7 & 35.5 & 52.3 & 61.7 \\
\hline 14.00 & 35.5 & 33.8 & 37.7 & 40.8 & 33.6 & 35.5 & 51.6 & 60.6 \\
\hline 14.25 & 35.3 & 33.7 & 37.6 & 40.5 & 33.6 & 35.4 & 50.9 & 59.6 \\
\hline 14.50 & 35.0 & 33.6 & 37.4 & 40.2 & 33.5 & 35.3 & 50.2 & 58.5 \\
\hline 14.75 & 34.8 & 33.6 & 37.3 & 40.0 & 33.5 & 35.3 & 49.5 & 57.6 \\
\hline 15.00 & 34.6 & 33.5 & 37.2 & 39.7 & 33.4 & 35.2 & 48.9 & 56.6 \\
\hline 15.25 & 34.4 & 33.4 & 37.0 & 39.5 & 33.4 & 35.1 & 48.2 & 55.7 \\
\hline 15.50 & 34.2 & 33.3 & 36.9 & 39.2 & 33.3 & 35.1 & 47.6 & 54.8 \\
\hline 15.75 & 34.0 & 33.2 & 36.8 & 39.0 & 33.3 & 35.0 & 47.0 & 54.0 \\
\hline 16.00 & 33.8 & 33.1 & 36.6 & 38.8 & 33.2 & 34.9 & 46.5 & 53.1 \\
\hline 16.25 & 33.6 & 33.1 & 36.5 & 38.6 & 33.2 & 34.8 & 45.9 & 52.5 \\
\hline 16.50 & 33.4 & 32.9 & 36.4 & 38.4 & 33.1 & 34.7 & 45.4 & 51.6 \\
\hline 16.75 & 33.3 & 32.8 & 36.3 & 38.2 & 33.1 & 34.7 & 44.9 & 50.9 \\
\hline 17.00 & 33.1 & 32.7 & 36.1 & 38.0 & 33.0 & 34.6 & 44.4 & 50.2 \\
\hline 17.25 & 33.0 & 32.7 & 36.0 & 37.9 & 32.9 & 34.5 & 43.9 & 49.5 \\
\hline 17.50 & 32.8 & 32.6 & 35.9 & 37.7 & 32.9 & 34.5 & 43.4 & 48.9 \\
\hline 17.75 & 32.7 & 32.5 & 35.8 & 37.5 & 32.8 & 34.4 & 42.9 & 48.2 \\
\hline 18.00 & 32.6 & 32.5 & 35.6 & 37.3 & 32.8 & 34.3 & 42.5 & 47.6 \\
\hline 18.25 & 32.4 & 32.4 & 35.5 & 37.2 & 32.7 & 34.2 & 42.0 & 47.1 \\
\hline 18.50 & 32.3 & 32.3 & 35.4 & 37.0 & 32.7 & 34.1 & 41.6 & 46.5 \\
\hline 18.75 & 32.2 & 32.2 & 35.3 & 36.9 & 32.6 & 34.1 & 41.2 & 46.0 \\
\hline 19.00 & 32.1 & 32.2 & 35.2 & 36.7 & 32.5 & 34.0 & 40.8 & 45.4 \\
\hline 19.25 & 32.0 & 32.1 & 35.1 & 36.6 & 32.5 & 33.9 & 40.4 & 44.9 \\
\hline 19.50 & 31.9 & 32.0 & 35.0 & 36.4 & 32.4 & 33.8 & 40.0 & 44.4 \\
\hline 19.75 & 31.8 & 32.0 & 34.9 & 36.3 & 32.4 & 33.8 & 39.7 & 43.9 \\
\hline 20.00 & 31.7 & 31.9 & 34.8 & 36.2 & 32.3 & 33.7 & 39.3 & 43.5 \\
\hline
\end{tabular}


Table 7: Temp. $\left({ }^{\circ} \mathrm{C}\right)$ measured by thermocouples attached to substrate underside for Test 8

\begin{tabular}{|c|c|c|c|c|c|c|c|c|}
\hline Time (min.) & TC1 & TC2 & TC3 & $\mathrm{TC} 4^{\mathrm{a}}$ & TC5 & TC6 & TC7 & TC8 \\
\hline 0.00 & 26.6 & 26.2 & 27.3 & - & 25.7 & 26.5 & 24.1 & 25.3 \\
\hline 0.25 & 126.1 & 26.2 & 27.4 & - & 25.7 & 26.5 & 24.3 & 36.1 \\
\hline 0.50 & 150.4 & 26.3 & 27.4 & - & 25.7 & 26.6 & 43.2 & 114.9 \\
\hline 0.75 & 155.0 & 26.4 & 27.5 & - & 25.7 & 26.6 & 72.1 & 139.5 \\
\hline 1.00 & 155.7 & 26.4 & 27.7 & - & 25.8 & 26.7 & 93.2 & 148.4 \\
\hline 1.25 & 154.3 & 26.5 & 28.0 & - & 25.8 & 26.9 & 105.8 & 150.4 \\
\hline 1.50 & 151.7 & 26.8 & 28.5 & - & 25.9 & 27.3 & 112.2 & 149.8 \\
\hline 1.75 & 147.3 & 27.1 & 29.2 & - & 26.1 & 27.8 & 114.8 & 148.0 \\
\hline 2.00 & 141.2 & 27.5 & 30.1 & - & 26.3 & 28.4 & 115.6 & 146.0 \\
\hline 2.25 & 134.5 & 28.1 & 31.0 & - & 26.6 & 29.1 & 115.3 & 144.0 \\
\hline 2.50 & 127.7 & 28.7 & 31.9 & - & 27.0 & 29.8 & 114.2 & 142.0 \\
\hline 2.75 & 121.1 & 29.3 & 32.8 & - & 27.4 & 30.6 & 112.9 & 140.0 \\
\hline 3.00 & 114.9 & 30.0 & 33.6 & - & 27.8 & 31.3 & 111.5 & 138.2 \\
\hline 3.25 & 109.2 & 30.7 & 34.4 & - & 28.2 & 31.9 & 110.1 & 136.2 \\
\hline 3.50 & 103.9 & 31.2 & 35.1 & - & 28.7 & 32.5 & 108.6 & 134.2 \\
\hline 3.75 & 99.0 & 31.8 & 35.6 & - & 29.1 & 33.0 & 107.0 & 131.8 \\
\hline 4.00 & 94.5 & 32.3 & 36.1 & - & 29.5 & 33.5 & 105.4 & 129.2 \\
\hline 4.25 & 90.4 & 32.8 & 36.5 & - & 29.9 & 33.9 & 103.8 & 126.4 \\
\hline 4.50 & 86.7 & 33.2 & 36.9 & - & 30.3 & 34.3 & 102.1 & 123.4 \\
\hline 4.75 & 83.2 & 33.6 & 37.2 & - & 30.6 & 34.6 & 100.3 & 121.1 \\
\hline 5.00 & 80.0 & 34.0 & 37.4 & - & 30.9 & 34.9 & 98.8 & 121.2 \\
\hline 5.25 & 77.1 & 34.3 & 37.5 & - & 31.2 & 35.2 & 97.6 & 121.0 \\
\hline 5.50 & 74.4 & 34.5 & 37.6 & - & 31.4 & 35.4 & 96.4 & 119.8 \\
\hline 5.75 & 71.9 & 34.7 & 37.7 & - & 31.6 & 35.5 & 95.2 & 117.8 \\
\hline 6.00 & 69.6 & 34.9 & 37.8 & - & 31.9 & 35.7 & 93.9 & 115.3 \\
\hline 6.25 & 67.5 & 35.1 & 37.8 & - & 32.0 & 35.8 & 92.5 & 112.6 \\
\hline 6.50 & 65.5 & 35.2 & 37.8 & - & 32.2 & 35.9 & 91.1 & 109.7 \\
\hline 6.75 & 63.6 & 35.3 & 37.8 & - & 32.3 & 36.0 & 89.5 & 106.8 \\
\hline 7.00 & 61.9 & 35.4 & 37.7 & - & 32.4 & 36.0 & 87.9 & 103.9 \\
\hline 7.25 & 60.3 & 35.5 & 37.6 & - & 32.5 & 36.1 & 86.2 & 101.1 \\
\hline 7.50 & 58.8 & 35.5 & 37.6 & - & 32.6 & 36.1 & 84.5 & 98.4 \\
\hline 7.75 & 57.4 & 35.5 & 37.5 & - & 32.7 & 36.1 & 82.9 & 95.7 \\
\hline 8.00 & 56.1 & 35.5 & 37.4 & - & 32.8 & 36.1 & 81.3 & 93.1 \\
\hline 8.25 & 54.8 & 35.5 & 37.3 & - & 32.8 & 36.1 & 79.6 & 90.7 \\
\hline 8.50 & 53.6 & 35.5 & 37.2 & - & 32.8 & 36.1 & 78.0 & 88.3 \\
\hline 8.75 & 52.5 & 35.4 & 37.1 & - & 32.8 & 36.1 & 76.4 & 86.1 \\
\hline 9.00 & 51.5 & 35.4 & 37.0 & - & 32.8 & 36.1 & 74.9 & 84.0 \\
\hline 9.25 & 50.5 & 35.3 & 36.8 & - & 32.8 & 36.1 & 73.4 & 81.9 \\
\hline 9.50 & 49.6 & 35.3 & 36.7 & - & 32.8 & 36.0 & 72.0 & 79.9 \\
\hline 9.75 & 48.7 & 35.2 & 36.5 & - & 32.8 & 36.0 & 70.5 & 78.0 \\
\hline 10.00 & 47.8 & 35.1 & 36.4 & - & 32.8 & 36.0 & 69.1 & 76.2 \\
\hline
\end{tabular}


Table 7: (cont.)

\begin{tabular}{|c|c|c|c|c|c|c|c|c|}
\hline Time (min.) & $\mathrm{TC} 1$ & TC2 & TC3 & $\mathrm{TC} 4^{\mathrm{a}}$ & TC5 & TC6 & TC7 & TC8 \\
\hline 10.25 & 47.1 & 35.0 & 36.3 & - & 32.8 & 35.9 & 67.8 & 74.5 \\
\hline 10.50 & 46.3 & 34.9 & 36.1 & - & 32.8 & 35.9 & 66.5 & 72.8 \\
\hline 10.75 & 45.6 & 34.8 & 36.0 & - & 32.8 & 35.8 & 65.2 & 71.2 \\
\hline 11.00 & 44.9 & 34.7 & 35.9 & - & 32.8 & 35.8 & 64.0 & 69.7 \\
\hline 11.25 & 44.3 & 34.6 & 35.7 & - & 32.7 & 35.7 & 62.8 & 68.2 \\
\hline 11.50 & 43.6 & 34.5 & 35.6 & - & 32.7 & 35.7 & 61.7 & 66.8 \\
\hline 11.75 & 43.0 & 34.4 & 35.5 & - & 32.6 & 35.6 & 60.6 & 65.4 \\
\hline 12.00 & 42.5 & 34.3 & 35.3 & - & 32.6 & 35.6 & 59.5 & 64.2 \\
\hline 12.25 & 42.0 & 34.2 & 35.2 & - & 32.6 & 35.5 & 58.5 & 62.9 \\
\hline 12.50 & 41.4 & 34.0 & 35.1 & - & 32.5 & 35.4 & 57.5 & 61.7 \\
\hline 12.75 & 41.0 & 33.9 & 34.9 & - & 32.5 & 35.4 & 56.6 & 60.6 \\
\hline 13.00 & 40.5 & 33.8 & 34.8 & - & 32.4 & 35.3 & 55.7 & 59.6 \\
\hline 13.25 & 40.1 & 33.7 & 34.7 & - & 32.4 & 35.3 & 54.8 & 58.5 \\
\hline 13.50 & 39.6 & 33.6 & 34.5 & - & 32.3 & 35.2 & 53.9 & 57.5 \\
\hline 13.75 & 39.2 & 33.5 & 34.4 & - & 32.2 & 35.1 & 53.1 & 56.6 \\
\hline 14.00 & 38.8 & 33.3 & 34.3 & - & 32.2 & 35.1 & 52.2 & 55.7 \\
\hline 14.25 & 38.5 & 33.2 & 34.2 & - & 32.1 & 35.0 & 51.4 & 54.8 \\
\hline 14.50 & 38.1 & 33.1 & 34.0 & - & 32.1 & 34.9 & 50.7 & 53.9 \\
\hline 14.75 & 37.8 & 33.0 & 33.9 & - & 32.0 & 34.9 & 50.0 & 53.1 \\
\hline 15.00 & 37.4 & 32.9 & 33.8 & - & 32.0 & 34.8 & 49.2 & 52.3 \\
\hline 15.25 & 37.1 & 32.8 & 33.7 & - & 31.9 & 34.7 & 48.6 & 51.5 \\
\hline 15.50 & 36.8 & 32.6 & 33.6 & - & 31.9 & 34.7 & 47.9 & 50.8 \\
\hline 15.75 & 36.5 & 32.5 & 33.4 & - & 31.8 & 34.6 & 47.3 & 50.1 \\
\hline 16.00 & 36.2 & 32.4 & 33.4 & - & 31.7 & 34.5 & 46.7 & 49.4 \\
\hline 16.25 & 36.0 & 32.4 & 33.3 & - & 31.7 & 34.5 & 46.1 & 48.7 \\
\hline 16.50 & 35.7 & 32.3 & 33.2 & - & 31.6 & 34.4 & 45.5 & 48.1 \\
\hline 16.75 & 35.5 & 32.2 & 33.1 & - & 31.5 & 34.3 & 44.9 & 47.5 \\
\hline 17.00 & 35.2 & 32.1 & 33.0 & - & 31.5 & 34.2 & 44.4 & 46.9 \\
\hline 17.25 & 35.0 & 32.0 & 32.9 & - & 31.4 & 34.2 & 43.9 & 46.3 \\
\hline 17.50 & 34.7 & 31.9 & 32.8 & - & 31.4 & 34.1 & 43.3 & 45.7 \\
\hline 17.75 & 34.5 & 31.8 & 32.6 & - & 31.3 & 34.0 & 42.8 & 45.2 \\
\hline 18.00 & 34.3 & 31.7 & 32.5 & - & 31.3 & 34.0 & 42.3 & 44.6 \\
\hline 18.25 & 34.1 & 31.6 & 32.4 & - & 31.2 & 33.9 & 41.9 & 44.1 \\
\hline 18.50 & 33.9 & 31.5 & 32.4 & - & 31.1 & 33.8 & 41.4 & 43.6 \\
\hline 18.75 & 33.7 & 31.4 & 32.3 & - & 31.1 & 33.7 & 41.0 & 43.1 \\
\hline 19.00 & 33.5 & 31.3 & 32.2 & - & 31.0 & 33.7 & 40.6 & 42.6 \\
\hline 19.25 & 33.3 & 31.2 & 32.1 & - & 31.0 & 33.6 & 40.2 & 42.2 \\
\hline 19.50 & 33.1 & 31.1 & 32.0 & - & 30.9 & 33.5 & 39.8 & 41.7 \\
\hline 19.75 & 32.9 & 31.1 & 31.9 & - & 30.9 & 33.4 & 39.4 & 41.3 \\
\hline 20.00 & 32.8 & 31.0 & 31.8 & - & 30.8 & 33.4 & 39.0 & 40.9 \\
\hline
\end{tabular}

${ }^{\text {a }}$ TC4 failed during the test. 
Table 8: Temp. $\left({ }^{\circ} \mathrm{C}\right)$ measured by thermocouples attached to substrate underside for Test 9

\begin{tabular}{|c|c|c|c|c|c|c|c|c|}
\hline Time (min.) & TC1 & TC2 & TC3 & TC4 & TC5 & TC6 & TC7 & TC8 \\
\hline 0.00 & 28.4 & 28.1 & 29.1 & 30.1 & 27.6 & 28.4 & 26.2 & 27.6 \\
\hline 0.25 & 71.6 & 28.1 & 29.2 & 36.1 & 27.7 & 28.5 & 30.6 & 135.2 \\
\hline 0.50 & 99.6 & 28.2 & 29.3 & 50.4 & 27.7 & 28.5 & 47.3 & 166.3 \\
\hline 0.75 & 120.8 & 28.2 & 29.6 & 60.0 & 27.7 & 28.7 & 60.4 & 179.3 \\
\hline 1.00 & 133.1 & 28.3 & 29.9 & 65.0 & 27.8 & 29.2 & 70.9 & 186.4 \\
\hline 1.25 & 139.3 & 28.3 & 30.6 & 67.1 & 27.9 & 29.8 & 80.4 & 189.6 \\
\hline 1.50 & 141.4 & 28.4 & 31.5 & 67.8 & 28.0 & 30.7 & 88.6 & 191.3 \\
\hline 1.75 & 140.7 & 28.5 & 32.6 & 67.6 & 28.1 & 31.7 & 95.4 & 191.6 \\
\hline 2.00 & 138.0 & 28.6 & 33.7 & 67.0 & 28.2 & 32.7 & 100.8 & 189.9 \\
\hline 2.25 & 133.9 & 28.8 & 34.9 & 66.1 & 28.3 & 33.6 & 104.8 & 186.8 \\
\hline 2.50 & 129.0 & 29.0 & 36.1 & 65.0 & 28.5 & 34.5 & 107.5 & 183.0 \\
\hline 2.75 & 123.8 & 29.3 & 37.3 & 63.9 & 28.7 & 35.3 & 109.2 & 178.8 \\
\hline 3.00 & 118.5 & 29.6 & 38.4 & 62.8 & 29.0 & 36.0 & 110.0 & 174.4 \\
\hline 3.25 & 113.3 & 29.9 & 39.4 & 61.7 & 29.2 & 36.6 & 110.2 & 169.8 \\
\hline 3.50 & 108.3 & 30.2 & 40.3 & 60.6 & 29.5 & 37.1 & 109.9 & 165.1 \\
\hline 3.75 & 103.5 & 30.5 & 41.1 & 59.5 & 29.8 & 37.5 & 109.2 & 160.5 \\
\hline 4.00 & 99.0 & 30.8 & 41.8 & 58.5 & 30.0 & 37.9 & 108.2 & 155.9 \\
\hline 4.25 & 94.9 & 31.1 & 42.4 & 57.5 & 30.3 & 38.2 & 106.9 & 151.4 \\
\hline 4.50 & 90.9 & 31.4 & 42.8 & 56.5 & 30.5 & 38.4 & 105.4 & 147.0 \\
\hline 4.75 & 87.3 & 31.7 & 43.2 & 55.6 & 30.8 & 38.6 & 103.8 & 142.8 \\
\hline 5.00 & 83.9 & 32.0 & 43.5 & 54.7 & 31.0 & 38.8 & 102.2 & 138.6 \\
\hline 5.25 & 80.7 & 32.2 & 43.7 & 53.9 & 31.2 & 39.0 & 100.4 & 134.5 \\
\hline 5.50 & 77.7 & 32.4 & 43.8 & 53.1 & 31.5 & 39.1 & 98.7 & 130.7 \\
\hline 5.75 & 75.0 & 32.6 & 43.9 & 52.3 & 31.7 & 39.2 & 96.9 & 126.9 \\
\hline 6.00 & 72.4 & 32.8 & 44.0 & 51.6 & 31.9 & 39.3 & 95.1 & 123.3 \\
\hline 6.25 & 70.0 & 33.0 & 44.0 & 51.0 & 32.0 & 39.3 & 93.3 & 119.9 \\
\hline 6.50 & 67.8 & 33.1 & 43.9 & 50.3 & 32.2 & 39.3 & 91.5 & 116.6 \\
\hline 6.75 & 65.7 & 33.3 & 43.9 & 49.7 & 32.4 & 39.4 & 89.7 & 113.4 \\
\hline 7.00 & 63.7 & 33.4 & 43.8 & 49.1 & 32.5 & 39.4 & 88.0 & 110.3 \\
\hline 7.25 & 61.9 & 33.5 & 43.6 & 48.5 & 32.7 & 39.4 & 86.3 & 107.4 \\
\hline 7.50 & 60.2 & 33.6 & 43.5 & 47.9 & 32.8 & 39.4 & 84.6 & 104.6 \\
\hline 7.75 & 58.6 & 33.6 & 43.3 & 47.4 & 33.0 & 39.4 & 83.0 & 101.9 \\
\hline 8.00 & 57.1 & 33.7 & 43.1 & 46.9 & 33.1 & 39.3 & 81.4 & 99.3 \\
\hline 8.25 & 55.7 & 33.7 & 43.0 & 46.5 & 33.2 & 39.3 & 79.8 & 96.8 \\
\hline 8.50 & 54.4 & 33.7 & 42.7 & 46.0 & 33.3 & 39.3 & 78.3 & 94.4 \\
\hline 8.75 & 53.1 & 33.7 & 42.5 & 45.6 & 33.4 & 39.2 & 76.8 & 92.1 \\
\hline 9.00 & 52.0 & 33.7 & 42.3 & 45.1 & 33.5 & 39.2 & 75.4 & 89.9 \\
\hline 9.25 & 50.9 & 33.7 & 42.2 & 44.7 & 33.6 & 39.1 & 73.9 & 87.8 \\
\hline 9.50 & 49.9 & 33.7 & 41.9 & 44.3 & 33.6 & 39.1 & 72.6 & 85.7 \\
\hline 9.75 & 48.9 & 33.7 & 41.7 & 43.9 & 33.7 & 39.0 & 71.2 & 83.7 \\
\hline
\end{tabular}


Table 8: (cont.)

\begin{tabular}{|c|c|c|c|c|c|c|c|c|}
\hline Time (min.) & TC1 & TC2 & TC3 & TC4 & TC5 & TC6 & TC7 & TC8 \\
\hline 10.00 & 48.0 & 33.6 & 41.5 & 43.6 & 33.7 & 38.9 & 69.9 & 81.9 \\
\hline 10.25 & 47.2 & 33.6 & 41.3 & 43.2 & 33.8 & 38.9 & 68.7 & 80.0 \\
\hline 10.50 & 46.4 & 33.6 & 41.1 & 42.9 & 33.8 & 38.8 & 67.4 & 78.3 \\
\hline 10.75 & 45.6 & 33.5 & 40.9 & 42.6 & 33.9 & 38.7 & 66.2 & 76.6 \\
\hline 11.00 & 44.9 & 33.5 & 40.7 & 42.3 & 33.9 & 38.7 & 65.1 & 75.0 \\
\hline 11.25 & 44.2 & 33.4 & 40.5 & 42.0 & 33.9 & 38.6 & 64.0 & 73.5 \\
\hline 11.50 & 43.6 & 33.4 & 40.3 & 41.7 & 34.0 & 38.5 & 62.9 & 72.0 \\
\hline 11.75 & 43.0 & 33.3 & 40.1 & 41.4 & 34.0 & 38.4 & 61.9 & 70.5 \\
\hline 12.00 & 42.4 & 33.2 & 39.9 & 41.2 & 34.0 & 38.3 & 60.8 & 69.1 \\
\hline 12.25 & 41.9 & 33.2 & 39.7 & 40.9 & 34.0 & 38.2 & 59.9 & 67.8 \\
\hline 12.50 & 41.4 & 33.1 & 39.5 & 40.6 & 34.0 & 38.1 & 58.9 & 66.5 \\
\hline 12.75 & 40.9 & 33.1 & 39.3 & 40.4 & 34.0 & 38.1 & 58.0 & 65.3 \\
\hline 13.00 & 40.4 & 33.0 & 39.1 & 40.2 & 34.0 & 38.0 & 57.1 & 64.2 \\
\hline 13.25 & 40.0 & 32.9 & 38.9 & 39.9 & 34.0 & 37.8 & 56.2 & 63.0 \\
\hline 13.50 & 39.6 & 32.9 & 38.7 & 39.7 & 34.0 & 37.7 & 55.4 & 61.9 \\
\hline 13.75 & 39.2 & 32.8 & 38.5 & 39.5 & 34.0 & 37.6 & 54.6 & 60.8 \\
\hline 14.00 & 38.8 & 32.8 & 38.4 & 39.3 & 34.0 & 37.5 & 53.8 & 59.8 \\
\hline 14.25 & 38.4 & 32.7 & 38.2 & 39.1 & 33.9 & 37.4 & 53.0 & 58.8 \\
\hline 14.50 & 38.1 & 32.6 & 38.0 & 38.9 & 33.9 & 37.3 & 52.3 & 57.9 \\
\hline 14.75 & 37.7 & 32.6 & 37.8 & 38.7 & 33.9 & 37.2 & 51.6 & 57.0 \\
\hline 15.00 & 37.4 & 32.5 & 37.7 & 38.5 & 33.9 & 37.1 & 50.9 & 56.1 \\
\hline 15.25 & 37.1 & 32.4 & 37.5 & 38.4 & 33.8 & 37.1 & 50.2 & 55.2 \\
\hline 15.50 & 36.8 & 32.4 & 37.3 & 38.2 & 33.8 & 36.9 & 49.6 & 54.4 \\
\hline 15.75 & 36.6 & 32.3 & 37.2 & 38.0 & 33.8 & 36.9 & 48.9 & 53.6 \\
\hline 16.00 & 36.3 & 32.2 & 37.0 & 37.9 & 33.7 & 36.7 & 48.3 & 52.9 \\
\hline 16.25 & 36.1 & 32.2 & 36.8 & 37.7 & 33.7 & 36.6 & 47.7 & 52.1 \\
\hline 16.50 & 35.8 & 32.1 & 36.7 & 37.6 & 33.7 & 36.5 & 47.2 & 51.4 \\
\hline 16.75 & 35.6 & 32.0 & 36.5 & 37.4 & 33.6 & 36.4 & 46.6 & 50.7 \\
\hline 17.00 & 35.4 & 32.0 & 36.4 & 37.3 & 33.6 & 36.3 & 46.1 & 50.1 \\
\hline 17.25 & 35.2 & 31.9 & 36.3 & 37.1 & 33.5 & 36.2 & 45.5 & 49.4 \\
\hline 17.50 & 35.0 & 31.8 & 36.1 & 37.0 & 33.5 & 36.1 & 45.0 & 48.8 \\
\hline 17.75 & 34.8 & 31.7 & 36.0 & 36.9 & 33.4 & 36.0 & 44.5 & 48.2 \\
\hline 18.00 & 34.6 & 31.7 & 35.8 & 36.7 & 33.4 & 35.9 & 44.0 & 47.6 \\
\hline 18.25 & 34.4 & 31.6 & 35.7 & 36.6 & 33.3 & 35.8 & 43.6 & 47.1 \\
\hline 18.50 & 34.2 & 31.5 & 35.6 & 36.5 & 33.3 & 35.7 & 43.1 & 46.5 \\
\hline 18.75 & 34.1 & 31.5 & 35.5 & 36.4 & 33.3 & 35.6 & 42.7 & 46.0 \\
\hline 19.00 & 33.9 & 31.4 & 35.3 & 36.3 & 33.2 & 35.5 & 42.3 & 45.5 \\
\hline 19.25 & 33.7 & 31.4 & 35.2 & 36.2 & 33.2 & 35.4 & 41.9 & 45.0 \\
\hline 19.50 & 33.6 & 31.3 & 35.1 & 36.1 & 33.1 & 35.3 & 41.5 & 44.6 \\
\hline 19.75 & 33.4 & 31.2 & 35.0 & 36.0 & 33.1 & 35.2 & 41.1 & 44.1 \\
\hline 20.00 & 33.3 & 31.2 & 34.9 & 35.9 & 33.0 & 35.1 & 40.8 & 43.7 \\
\hline
\end{tabular}




\subsection{Temperature measurements of salt and substrate surface by IR camera}

The temperatures of the salt and substrate surface during and after molten salt spreading were recorded with an IR camera. Image processing techniques in MATLAB® were used to distinguish the salt from the substrate in the IR video frames; a description of the method is provided in Section 3.1.2.3 of Thomas and Jackson, 2021. The IR video records the temperature of each pixel in the field of view of the camera (resolution of $348 \times 464$ pixels) at a rate of 30 frames per second. The IR video data files have been exported as .csv files and are available from the authors upon reasonable request. These data files have been processed to correct for the temperature measurement of stainless steel (as described in Appendix B of Thomas and Jackson, 2021) and consist of two sets of data. The first dataset contains separate files of the salt surface temperature and the substrate surface temperature that were collected at 30 frames per second for the first 17 seconds of the test. The salt and substrate are distinguished in their respective files by setting the pixel values of the other material to "NaN". The second dataset contains separate files of the salt surface temperature and the substrate surface temperature that were collected at 5 frames per second to provide cooling information. The total number of frames available varies by test. A file that shows the time stamp for each frame is also provided for each dataset.

The pixel coordinates of the corners of the stainless steel substrate are provided in Table 9. A schematic that identifies the pixel coordinates relative to the direction of the salt flow is provided in Figure 2. It is important to note that the temperature measurement of the stainless steel surface using the IR camera is highly sensitive to the temperature of surrounding objects due to the low emissivity of stainless steel. Although accounted for in image processing, the reflection of radiation from adjacent objects may be apparent on the stainless steel surface in the data files. The temperature value in the data file was set to "NaN" if the corrected temperature of the stainless steel surface was below $22^{\circ} \mathrm{C}$. The salt temperature measured by the IR camera is not sensitive to the temperature of adjacent objects due to the high emissivity of the salt.

Table 9: The coordinates of the corners of the stainless steel substrate in pixels

\begin{tabular}{ccccccccc}
\hline Test & $\mathrm{x}_{1}$ & $\mathrm{y}_{1}$ & $\mathrm{x}_{2}$ & $\mathrm{y}_{2}$ & $\mathrm{x}_{3}$ & $\mathrm{y}_{3}$ & $\mathrm{x}_{4}$ & $\mathrm{y}_{4}$ \\
\hline 1 & 128 & 22 & 316 & 15 & 120 & 415 & 299 & 430 \\
2 & 74 & 29 & 262 & 24 & 77 & 431 & 256 & 436 \\
3 & 41 & 17 & 236 & 14 & 48 & 439 & 233 & 436 \\
$4^{\mathrm{a}}$ & 118 & 13 & 300 & 13 & 127 & 413 & 299 & 413 \\
$5^{\mathrm{b}}$ & 22 & 1 & 268 & 1 & 28 & 464 & 250 & 464 \\
6 & 103 & 26 & 281 & 28 & 110 & 431 & 281 & 436 \\
$7^{\mathrm{c}, \mathrm{d}}$ & 70 & 9 & 261 & 3 & 84 & 420 & 255 & 422 \\
$8^{\mathrm{c}, \mathrm{d}}$ & 49 & 37 & 225 & 37 & 49 & 453 & 225 & 453 \\
$9^{\mathrm{d}}$ & 159 & 42 & 348 & 44 & 155 & 443 & 333 & 448 \\
\hline
\end{tabular}

${ }^{a}$ A platinum foil is blocking the bottom 1 in. of the substrate from the camera field of view.

${ }^{\mathrm{b}}$ The bottom $1.5 \mathrm{in}$. of the substrate is out of the camera field of view and the edge of the stainless steel substrate is provided instead.

${ }^{\mathrm{c}}$ The provided coordinates of the corners of the stainless steel substrate are estimates because the boundary could not be distinguished in the IR video.

${ }^{d}$ The stainless steel surface temperature was not measured because a higher temperature calibration setting was required to measure the salt temperature with the IR camera. 


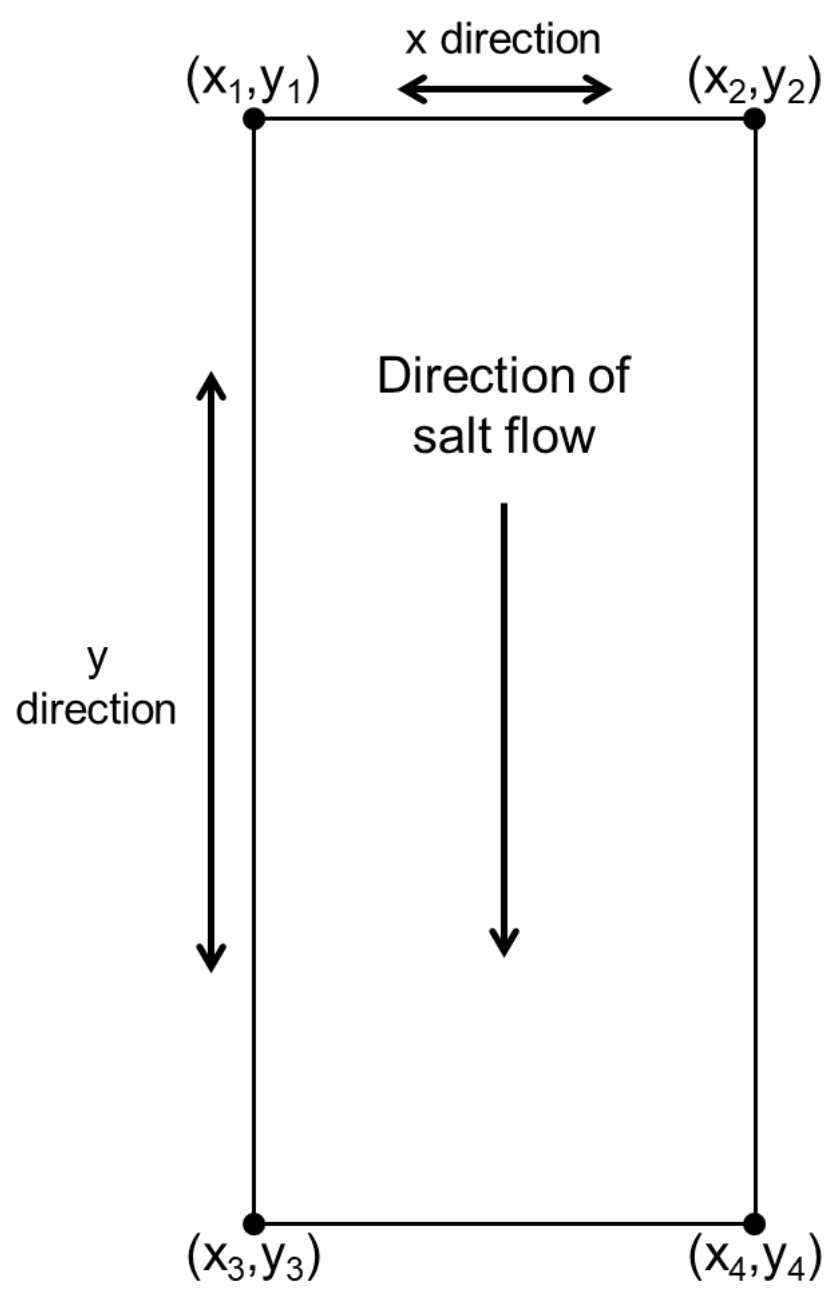

Figure 2: Schematic of the coordinates of the stainless steel substrate boundary relative to the direction of the salt flow.

A summary of the pixel coordinates in the IR video frames that provide locations of the thermocouples attached to the substrate underside for each test are available upon reasonable request. The temperature information obtained from the thermocouples attached to the substrate underside surface and from the IR camera of the substrate upper surface or the salt surface at the same location could be used for heat transfer analysis.

\subsection{Leading edge and covered area as a function of time}

The leading edge and covered area of the salt on the substrate for each still frame recorded by the IR camera were determined using the methods described in Section 3.1.2.3 in Thomas and Jackson, 2021 and are provided for each test in Table 10 and Table 11, respectively. 
Table 10: Leading edge position $(\mathrm{mm})$ of molten salt spreading on stainless steel substrate

\begin{tabular}{|c|c|c|c|c|c|c|c|c|c|}
\hline \multirow[b]{2}{*}{ Time (ms) } & \multicolumn{9}{|c|}{ Test Number } \\
\hline & 1 & 2 & 3 & $4^{a}$ & $5^{b}$ & 6 & 7 & 8 & 9 \\
\hline 0 & 0 & 0 & 0 & - & 0 & 0 & 0 & 0 & 0 \\
\hline 33 & 1.4 & 15.4 & 11.9 & - & 4.4 & 1.4 & 8.0 & 5.4 & 1.4 \\
\hline 66 & 5.8 & 19.7 & 14.5 & - & 12.2 & 5.7 & 14.7 & 12.2 & 2.9 \\
\hline 100 & 10.1 & 29.5 & 18.5 & - & 15.5 & 8.5 & 16.0 & 15.0 & 5.7 \\
\hline 133 & 14.4 & 37.9 & 21.1 & - & 21.0 & 12.7 & 21.4 & 17.7 & 5.7 \\
\hline 166 & 18.7 & 46.3 & 22.4 & - & 24.4 & 18.4 & 24.1 & 17.7 & 10.0 \\
\hline 200 & 21.6 & 51.9 & 26.4 & - & 26.6 & 22.6 & 28.1 & 20.4 & 15.7 \\
\hline 233 & 25.9 & 60.4 & 29.0 & - & 32.1 & 28.3 & 32.1 & 23.1 & 20.0 \\
\hline 266 & 30.2 & 68.8 & 33.0 & - & 35.4 & 34.0 & 37.4 & 25.8 & 27.2 \\
\hline 300 & 33.1 & 77.2 & 36.9 & - & 39.9 & 39.6 & 42.8 & 28.6 & 32.9 \\
\hline 333 & 37.4 & 87.0 & 39.5 & - & 44.3 & 45.3 & 49.5 & 32.6 & 40.0 \\
\hline 366 & 43.2 & 98.3 & 42.2 & - & 50.9 & 50.9 & 56.2 & 36.7 & 48.6 \\
\hline 400 & 47.5 & 109.5 & 46.1 & - & 56.5 & 56.6 & 62.8 & 40.8 & 57.2 \\
\hline 433 & 51.8 & 120.7 & 50.1 & - & 64.2 & 107.5 & 69.5 & 46.2 & 64.4 \\
\hline 466 & 57.6 & 134.8 & 54.0 & - & 72.0 & 67.9 & 77.5 & 50.3 & 72.9 \\
\hline 500 & 63.4 & 134.8 & 58.0 & - & 79.7 & 75.0 & 85.6 & 57.1 & 81.5 \\
\hline 533 & 79.2 & 147.4 & 61.9 & - & 88.6 & 82.1 & 92.3 & 62.6 & 90.1 \\
\hline 566 & 73.4 & 161.5 & 67.2 & - & 97.4 & 89.1 & 101.6 & 66.6 & 98.7 \\
\hline 600 & 79.2 & 175.5 & 71.2 & - & 106.3 & 96.2 & 111.0 & 72.1 & 107.3 \\
\hline 633 & 85.0 & 190.9 & 76.4 & - & 117.3 & 103.3 & 120.3 & 77.5 & 117.3 \\
\hline 666 & 92.2 & 206.4 & 81.7 & - & 128.4 & 103.3 & 129.7 & 84.3 & 127.3 \\
\hline 700 & 97.9 & 221.8 & 87.0 & - & 140.6 & 111.8 & 140.4 & 89.8 & 137.3 \\
\hline 733 & 103.7 & 235.9 & 92.3 & - & 151.7 & 120.3 & 151.1 & 96.6 & 147.3 \\
\hline 766 & 110.9 & 251.3 & 98.9 & - & 163.8 & 130.2 & 161.8 & 103.4 & 157.3 \\
\hline 800 & 118.1 & 268.2 & 105.4 & - & 176.0 & 138.7 & 171.1 & 108.8 & 168.7 \\
\hline 833 & 125.3 & 285.0 & 112.0 & - & 189.3 & 148.6 & 181.8 & 115.6 & 180.2 \\
\hline 866 & 132.5 & 301.9 & 119.9 & - & 202.6 & 157.1 & 192.5 & 123.8 & 191.6 \\
\hline 900 & 139.7 & 320.1 & 126.5 & - & 217.0 & 167.0 & 203.2 & 130.6 & 203.1 \\
\hline 933 & 146.9 & 337.0 & 133.1 & - & 232.5 & 176.9 & 215.3 & 137.4 & 214.5 \\
\hline 966 & 154.1 & 355.2 & 141.0 & - & 248.0 & 188.2 & 226.0 & 145.5 & 225.9 \\
\hline 999 & 161.3 & 374.9 & 148.9 & - & 263.5 & 198.1 & 236.7 & 152.3 & 238.8 \\
\hline 1033 & 168.5 & 393.1 & 155.5 & - & 280.1 & 209.4 & 248.7 & 160.5 & 250.3 \\
\hline 1066 & 177.1 & 411.4 & 163.4 & - & 296.7 & 220.7 & 259.4 & 168.6 & 263.1 \\
\hline 1099 & 185.8 & 431.0 & 171.3 & - & 313.3 & 232.1 & 271.4 & 175.4 & 276.0 \\
\hline 1133 & 193.0 & 450.7 & 179.3 & - & 329.9 & 243.4 & 282.1 & 183.6 & 287.4 \\
\hline 1166 & 201.6 & 468.9 & 185.8 & - & 347.6 & 254.7 & 294.1 & 190.4 & 300.3 \\
\hline 1199 & 210.2 & 490.0 & 193.8 & - & 365.3 & 266.0 & 304.8 & 197.2 & 313.2 \\
\hline 1233 & 218.9 & 509.7 & 203.0 & - & 384.1 & 278.8 & 315.5 & 205.4 & 326.0 \\
\hline 1266 & 227.5 & 530.7 & 210.9 & - & 401.8 & 291.5 & 326.2 & 212.2 & 338.9 \\
\hline 1299 & 237.6 & 546.2 & 218.8 & - & - & 304.2 & 336.9 & 219.0 & 351.8 \\
\hline 1333 & 246.2 & - & 226.7 & - & 438.4 & 315.6 & 347.6 & 225.8 & 364.7 \\
\hline 1366 & 256.3 & - & 234.6 & - & 456.1 & 328.3 & 358.3 & 233.9 & 379.0 \\
\hline 1399 & 266.4 & - & 234.6 & - & 473.8 & 339.6 & 367.7 & 243.4 & 393.3 \\
\hline
\end{tabular}


Table 10: (cont.)

\begin{tabular}{|c|c|c|c|c|c|c|c|c|c|}
\hline \multirow[b]{2}{*}{ Time (ms) } & \multicolumn{9}{|c|}{ Test Number } \\
\hline & 1 & 2 & 3 & $4^{a}$ & $5^{b}$ & 6 & 7 & 8 & 9 \\
\hline 1433 & 276.5 & - & 243.8 & - & 491.5 & 352.3 & 377.0 & 250.2 & 407.6 \\
\hline 1466 & 288.0 & - & 253.1 & - & - & 365.1 & 386.4 & 259.8 & 420.4 \\
\hline 1499 & 298.1 & - & 261.0 & - & 502.6 & 377.8 & 394.4 & 267.9 & 436.2 \\
\hline 1533 & 309.6 & - & 270.2 & - & - & 390.5 & 403.8 & 267.9 & 450.5 \\
\hline 1566 & 319.7 & - & 278.1 & - & - & 403.3 & 410.5 & 276.1 & 464.8 \\
\hline 1599 & 331.2 & - & 286.0 & - & - & 417.4 & 418.5 & 285.6 & 479.1 \\
\hline 1633 & 341.3 & - & 295.2 & - & - & 430.2 & 423.8 & 293.8 & 493.4 \\
\hline 1666 & 352.8 & - & 304.5 & - & - & 444.3 & 430.5 & 301.9 & 507.7 \\
\hline 1699 & 364.3 & - & 312.4 & - & - & 458.5 & 435.9 & 310.1 & 522.0 \\
\hline 1733 & 374.4 & - & 321.6 & - & - & 472.6 & 439.9 & 318.2 & 536.3 \\
\hline 1766 & 385.9 & - & 330.8 & - & - & 486.8 & 445.2 & 326.4 & 550.6 \\
\hline 1799 & 397.4 & - & 338.7 & - & - & 500.9 & 449.2 & 334.6 & - \\
\hline 1833 & 409.0 & - & 348.0 & - & - & 513.6 & 457.3 & 342.7 & - \\
\hline 1866 & 421.9 & - & 355.9 & - & - & 529.2 & 469.3 & 350.9 & - \\
\hline 1899 & 433.4 & - & 363.8 & - & - & 540.5 & 481.3 & 359.0 & - \\
\hline 1933 & 446.4 & - & 373.0 & - & - & - & 494.7 & 368.6 & - \\
\hline 1966 & 457.9 & - & 382.2 & - & - & - & 506.7 & 376.7 & - \\
\hline 1999 & 469.4 & - & 391.5 & - & - & - & 520.1 & 384.9 & - \\
\hline 2033 & 482.4 & - & 400.7 & - & - & - & 530.8 & 394.4 & - \\
\hline 2066 & 492.5 & - & 409.9 & - & - & - & - & 402.6 & - \\
\hline 2099 & 504.0 & - & 419.1 & - & - & - & - & 412.1 & - \\
\hline 2133 & 514.1 & - & 427.0 & - & - & - & - & 420.2 & - \\
\hline 2166 & 524.2 & - & 434.9 & - & - & - & - & 428.4 & - \\
\hline 2199 & 534.2 & - & 444.2 & - & - & - & - & 436.6 & - \\
\hline 2233 & 544.3 & - & 452.1 & - & - & - & - & 444.7 & - \\
\hline 2266 & - & - & 460.0 & - & - & - & - & 452.9 & - \\
\hline 2299 & - & - & 467.9 & - & - & - & - & 461.0 & - \\
\hline 2333 & - & - & 475.8 & - & - & - & - & 469.2 & - \\
\hline 2366 & - & - & 483.7 & - & - & - & - & 477.4 & - \\
\hline 2399 & - & - & 491.6 & - & - & - & - & 485.5 & - \\
\hline 2433 & - & - & 499.5 & - & - & - & - & 492.3 & - \\
\hline 2466 & - & - & 506.1 & - & - & - & - & 500.5 & - \\
\hline 2499 & - & - & 512.7 & - & - & - & - & 507.3 & - \\
\hline 2533 & - & - & 519.3 & - & - & - & - & 514.1 & - \\
\hline 2566 & - & - & 527.2 & - & - & - & - & 520.9 & - \\
\hline 2599 & - & - & 535.1 & - & - & - & - & 527.7 & - \\
\hline 2633 & - & - & 545.7 & - & - & - & - & 533.1 & - \\
\hline 2666 & - & - & - & - & - & - & - & 539.9 & - \\
\hline 2699 & - & - & - & - & - & - & - & 545.4 & - \\
\hline 2733 & - & - & - & - & - & - & - & 550.8 & - \\
\hline 2766 & - & - & - & - & - & - & - & 557.6 & - \\
\hline
\end{tabular}

a The leading edge data was not usable for this test.

$\mathrm{b}$ The final $1.5 \mathrm{in}$. of the substrate was out of the field of view of the IR camera. 
Table 11: Covered area $\left(\mathrm{mm}^{2}\right)$ of molten salt spreading on stainless steel substrate

\begin{tabular}{|c|c|c|c|c|c|c|c|c|c|}
\hline \multirow[b]{2}{*}{ Time (ms) } & \multicolumn{9}{|c|}{ Test Number } \\
\hline & 1 & 2 & 3 & $4^{a}$ & $5^{b}$ & 6 & 7 & 8 & 9 \\
\hline 0 & 0 & 0 & 0 & 0 & 0 & 0 & 0 & 0 & 0 \\
\hline 33 & 19.9 & 271.0 & 147.7 & 82.9 & 53.5 & 31.3 & 60.4 & 43.0 & 35.8 \\
\hline 66 & 105.3 & 403.1 & 187.3 & 145.5 & 131.0 & 116.3 & 166.6 & 101.9 & 73.1 \\
\hline 100 & 170.8 & 545.4 & 248.6 & 269.0 & 203.2 & 197.7 & 187.8 & 150.7 & 135.1 \\
\hline 133 & 232.0 & 726.9 & 307.1 & 382.9 & 285.0 & 280.3 & 260.0 & 203.1 & 135.1 \\
\hline 166 & 339.2 & 919.3 & 351.4 & 480.1 & 372.7 & 388.3 & 326.8 & 219.0 & 223.5 \\
\hline 200 & 418.1 & 1108.6 & 457.7 & 628.9 & 434.7 & 462.2 & 377.8 & 262.4 & 375.7 \\
\hline 233 & 503.2 & 1287.5 & 502.9 & 776.9 & 564.3 & 567.7 & 478.2 & 333.0 & 565.2 \\
\hline 266 & 601.1 & 1464.6 & 566.3 & 935.8 & 664.9 & 679.8 & 555.9 & 396.3 & 742.5 \\
\hline 300 & 676.5 & 1667.2 & 664.0 & 1120.6 & 761.5 & 779.5 & 622.3 & 489.2 & 883.3 \\
\hline 333 & 764.9 & 1878.2 & 733.8 & 1296.3 & 880.7 & 897.3 & 675.5 & 591.1 & 1028.7 \\
\hline 366 & 874.5 & 2065.3 & 805.1 & 1467.1 & 999.7 & 1013.3 & 789.9 & 679.9 & 1174.9 \\
\hline 400 & 972.9 & 2275.5 & 913.0 & 1660.8 & 1114.1 & 1140.0 & 847.5 & 790.5 & 1350.4 \\
\hline 433 & 1068.4 & 2522.9 & 1036.1 & 1829.8 & 1262.7 & 1277.4 & 904.2 & 910.3 & 1507.8 \\
\hline 466 & 1179.4 & 2770.2 & 1143.3 & 2046.6 & 1416.5 & 1424.9 & 988.0 & 1007.6 & 1648.6 \\
\hline 500 & 1300.2 & 2770.2 & 1256.4 & 2267.2 & 1585.7 & 1602.7 & 1055.6 & 1107.7 & 1791.9 \\
\hline 533 & 1398.6 & 3012.3 & 1366.0 & 2432.9 & 1752.4 & 1739.9 & 1127.6 & 1183.4 & 1964.1 \\
\hline 566 & 1520.5 & 3212.3 & 1498.3 & 2603.9 & 1939.9 & 1873.0 & 1215.0 & 1280.2 & 2107.4 \\
\hline 600 & 1651.6 & 3468.6 & 1635.5 & 2829.5 & 2105.0 & 2085.6 & 1286.1 & 1396.6 & 2284.0 \\
\hline 633 & 1770.7 & 3674.5 & 1757.6 & 3029.7 & 2293.9 & 2251.2 & 1357.5 & 1510.2 & 2434.6 \\
\hline 666 & 1897.5 & 3905.3 & 1885.2 & 3233.8 & 2448.6 & 2251.2 & 1433.0 & 1596.2 & 2650.1 \\
\hline 700 & & & 2055.4 & & 2602.7 & & & 1700.2 & 2837.0 \\
\hline 733 & 2145.8 & 4339.5 & 2198.6 & 3655.7 & 2768.6 & 2627.2 & 1593.6 & 1836.1 & 3032.2 \\
\hline 766 & 2263.6 & 4548.7 & 2338.2 & 3901.7 & 2910.1 & 2808.0 & 1648.8 & 1973.4 & 3186.7 \\
\hline 800 & 2391.4 & 4824.6 & 2491.6 & 4103.3 & 3027.9 & 2961.0 & 1710.7 & 2121.8 & 3345.4 \\
\hline 833 & 2541.6 & 5078.0 & 2662.3 & 4103.3 & 3199.9 & 3132.3 & 1763.5 & 2235.4 & 3463.6 \\
\hline 866 & 2647.1 & 5244.2 & 2839.6 & 4292.6 & 3382.4 & 3322.4 & 1820.8 & 2344.4 & 3638.1 \\
\hline 900 & 2769.5 & 5492.4 & 2985.1 & 4483.1 & 3571.8 & 3490.1 & 1904.2 & 2429.3 & 3752.6 \\
\hline 933 & 2903.1 & & & 4706.8 & 3708.1 & 3658.3 & 2011.5 & 2560.4 & 3887.2 \\
\hline 966 & 3038.2 & 5908.0 & 3252.3 & 4924.4 & 3819.1 & 3823.4 & 2058.1 & 2635.1 & 3980.5 \\
\hline 999 & 3168.3 & 6124.3 & 3453.3 & 5124.1 & 3967.2 & 4010.2 & 2141.7 & 2723.7 & 4060.6 \\
\hline 1033 & 3318.1 & 6352.3 & 3584.3 & 5308.8 & 4107.0 & 4178.4 & 2245.0 & 2794.0 & 4174.4 \\
\hline 1066 & 3456.6 & 6555.4 & 3725.1 & 5485.8 & 4238.1 & 4239.7 & 2292.9 & 2861.4 & 4311.1 \\
\hline 1099 & 3603.5 & 6740.4 & 3885.6 & 5681.7 & 4349.8 & 4408.9 & 2329.3 & 2954.8 & 4447.7 \\
\hline 1133 & 3711.7 & 6957.0 & 4038.5 & 5857.1 & 4431.0 & 4548.7 & 2346.1 & 2959.4 & 4527.5 \\
\hline 1166 & 3866.5 & 7141.8 & 4190.7 & 6049.6 & 4538.2 & 4712.2 & 2368.7 & 3040.4 & 4603.2 \\
\hline 1199 & 4022.9 & 7375.9 & 4343.5 & 6248.8 & 4663.4 & 4855.9 & 2416.2 & 3122.3 & 4744.5 \\
\hline 1233 & 4130.3 & 7564.5 & 4528.9 & 6436.6 & 4732.8 & 4980.2 & 2440.2 & 3201.6 & 4780.8 \\
\hline 1266 & 4252.2 & 7777.8 & 4672.1 & 6629.4 & 4750.1 & 5106.8 & 2463.2 & 3280.0 & 4899.3 \\
\hline 1299 & 4431.2 & 7952.4 & 4824.8 & 6820.3 & - & 5253.0 & 2512.2 & 3338.9 & 4926.0 \\
\hline 1333 & 4528.0 & - & 4993.2 & 6976.1 & 4858.8 & 5366.7 & 2550.8 & 3386.5 & 4964.9 \\
\hline 1366 & 4703.7 & - & 5113.3 & 7168.1 & 4926.6 & 5442.4 & 2596.1 & 3462.9 & 5042.2 \\
\hline 1399 & 4883.5 & - & 5113.3 & 7299.6 & 4932.3 & 5616.3 & 2634.2 & 3564.8 & 5095.3 \\
\hline
\end{tabular}


Table 11: (cont.)

\begin{tabular}{|c|c|c|c|c|c|c|c|c|c|}
\hline \multirow[b]{2}{*}{ Time (ms) } & \multicolumn{9}{|c|}{ Test Number } \\
\hline & 1 & 2 & 3 & $4^{a}$ & $5^{b}$ & 6 & 7 & 8 & 9 \\
\hline 1433 & 5020.3 & - & 5263.7 & 7483.3 & 5020.4 & 5772.1 & 2673.1 & 3624.8 & 5164.3 \\
\hline 1466 & 5166.4 & - & 5421.9 & 7596.0 & - & 5881.6 & 2722.4 & 3701.9 & 5238.9 \\
\hline 1499 & 5356.5 & - & 5546.7 & 7717.7 & 4862.5 & 6030.2 & 2748.0 & 3798.0 & 5315.4 \\
\hline 1533 & 5467.2 & - & 5685.0 & 7806.0 & - & 6149.1 & 2767.2 & 3798.0 & 5384.1 \\
\hline 1566 & 5626.3 & - & 5846.3 & 7918.7 & - & 6312.1 & 2820.6 & 3813.9 & 5465.5 \\
\hline 1599 & 5778.4 & - & 5980.0 & 8033.4 & - & 6467.6 & 2866.4 & 3890.0 & 5487.8 \\
\hline 1633 & 5920.4 & - & 6135.4 & 8147.3 & - & 6555.2 & 2912.6 & 3970.0 & 5478.5 \\
\hline 1666 & 6041.1 & - & 6260.4 & 8251.5 & - & 6696.3 & 2914.3 & 4005.0 & 5520.7 \\
\hline 1699 & 6189.6 & - & 6382.5 & 8333.6 & - & 6812.3 & 2924.6 & 4051.0 & 5532.4 \\
\hline 1733 & 6302.5 & - & 6518.0 & 8437.0 & - & 6896.0 & 2899.9 & 4088.5 & 5624.2 \\
\hline 1766 & 6462.7 & - & 6634.3 & - & - & 6972.0 & 2879.7 & 4150.1 & 5672.1 \\
\hline 1799 & 6590.3 & - & 6764.4 & - & - & 7089.3 & 2879.5 & 4175.7 & - \\
\hline 1833 & 6721.7 & - & 6879.4 & - & - & 7126.2 & 2938.8 & 4287.9 & - \\
\hline 1866 & 6853.0 & - & 7039.0 & - & - & 7189.0 & 3001.4 & 4307.7 & - \\
\hline 1899 & 6996.7 & - & 7180.7 & - & - & 7353.9 & 2974.5 & 4331.8 & - \\
\hline 1933 & 7122.6 & - & 7273.1 & - & - & - & 2967.5 & 4396.0 & - \\
\hline 1966 & 7253.4 & - & 7444.4 & - & - & - & 2961.4 & 4406.8 & - \\
\hline 1999 & 7364.1 & - & 7551.3 & - & - & - & 2928.1 & 4471.7 & - \\
\hline 2033 & 7485.4 & - & 7653.3 & - & - & - & 2978.0 & 4449.1 & - \\
\hline 2066 & 7592.1 & - & 7776.0 & - & - & - & - & 4480.6 & - \\
\hline 2099 & 7703.0 & - & 7883.8 & - & - & - & - & 4525.0 & - \\
\hline 2133 & 7812.9 & - & 7975.7 & - & - & - & - & 4548.5 & - \\
\hline 2166 & 7887.7 & - & 8075.2 & - & - & - & - & 4553.8 & - \\
\hline 2199 & 7999.2 & - & 8216.6 & - & - & - & - & 4564.8 & - \\
\hline 2233 & 8100.2 & - & 8302.8 & - & - & - & - & 4576.5 & - \\
\hline 2266 & - & - & 8412.9 & - & - & - & - & 4640.9 & - \\
\hline 2299 & - & - & 8514.3 & - & - & - & - & 4652.9 & - \\
\hline 2333 & - & - & 8592.4 & - & - & - & - & 4684.2 & - \\
\hline 2366 & - & - & 8686.9 & - & - & - & - & 4669.7 & - \\
\hline 2399 & - & - & 8775.9 & - & - & - & - & 4683.5 & - \\
\hline 2433 & - & - & 8847.4 & - & - & - & - & 4713.2 & - \\
\hline 2466 & - & - & 8925.5 & - & - & - & - & 4722.8 & - \\
\hline 2499 & - & - & 9026.2 & - & - & - & - & 4704.4 & - \\
\hline 2533 & - & - & 9075.7 & - & - & - & - & 4751.3 & - \\
\hline 2566 & - & - & 9171.5 & - & - & - & - & 4724.4 & - \\
\hline 2599 & - & - & 9256.8 & - & - & - & - & 4731.1 & - \\
\hline 2633 & - & - & 9329.6 & - & - & - & - & 4759.9 & - \\
\hline 2666 & - & - & - & - & - & - & - & 4760.8 & - \\
\hline 2699 & - & - & - & - & - & - & - & 4779.4 & - \\
\hline 2733 & - & - & - & - & - & - & - & 4797.6 & - \\
\hline 2766 & - & - & - & - & - & - & - & 4843.3 & - \\
\hline
\end{tabular}

${ }^{a} \mathrm{~A} 1$ in. wide platinum foil blocked the substrate bottom from the field of view of the IR camera.

$\mathrm{b}$ The final $1.5 \mathrm{in}$. of the substrate was out of the field of view of the IR camera. 


\subsection{Thermocouple measurements of atmosphere temperature above pool}

A thermocouple placed above the molten salt pool that forms at the bottom of the substrate was used to record the temperature of the atmosphere as a function of time since the salt was first poured onto the substrate. The location of the thermocouple was different in each test, and the thermocouple location relative to the center of the molten salt pool is provided in Table 12. The measurements from the thermocouple above the molten salt pool are provided in Table 13.

Table 12: Description of thermocouple location above molten salt pool ${ }^{\mathrm{a}}$

\begin{tabular}{cc}
\hline Test & $\begin{array}{c}\text { Thermocouple location relative to } \\
\text { molten salt pool }\end{array}$ \\
\hline 2 & $6 "$ " above \\
3 & 1 " above, 4 " off center of pool \\
5 & 6 " above \\
7 & 0.9 " above \\
8 & 2 " above, 1 " off center of pool \\
\hline
\end{tabular}

${ }^{a}$ Data from Tests 1, 4, 6, and 9 are unavailable.

Table 13: Temp. $\left({ }^{\circ} \mathrm{C}\right)$ measured by thermocouple above molten salt pool for spreading tests ${ }^{\mathrm{a}}$

\begin{tabular}{cccccc}
\hline Time (min.) & 2 & 3 & 5 & 7 & 8 \\
\hline 0.00 & 25.5 & 25.2 & 28.3 & 25.4 & 25.5 \\
0.25 & 25.6 & 25.4 & 28.4 & 37.3 & 25.9 \\
0.50 & 25.7 & 26.2 & 28.6 & 54.1 & 29.6 \\
0.75 & 25.8 & 26.8 & 28.8 & 69.7 & 33.3 \\
1.00 & 25.9 & 27.4 & 28.9 & 81.0 & 36.5 \\
1.25 & 25.9 & 28.0 & 29.0 & 93.0 & 39.6 \\
1.50 & 26.0 & 28.6 & 29.0 & 100.5 & 42.7 \\
1.75 & 26.0 & 29.4 & 29.1 & 106.4 & 45.8 \\
2.00 & 26.1 & 30.1 & 29.1 & 109.6 & 49.3 \\
2.25 & 26.1 & 30.9 & 29.2 & 111.2 & 51.8 \\
2.50 & 26.1 & 31.6 & 29.2 & 111.0 & 55.0 \\
2.75 & 26.1 & 32.4 & 29.2 & 110.4 & 56.4 \\
3.00 & 26.1 & 33.4 & 29.2 & 110.1 & 57.6 \\
3.25 & 26.2 & 34.4 & 29.2 & 109.0 & 59.1 \\
3.50 & 26.2 & 35.3 & 29.1 & 107.4 & 59.4 \\
3.75 & 26.2 & 36.4 & 29.1 & 105.8 & 59.7 \\
\hline
\end{tabular}

${ }^{a}$ Data from Tests 1, 4, 6, and 9 are unavailable. 
Table 13: (cont.)

\begin{tabular}{cccccc}
\hline Time (min.) & 2 & 3 & 5 & 7 & 8 \\
\hline 4.00 & 26.2 & 37.3 & 29.1 & 103.6 & 58.9 \\
4.25 & 26.2 & 38.1 & 29.1 & 101.5 & 58.3 \\
4.50 & 26.2 & 39.0 & 29.1 & 99.2 & 57.0 \\
4.75 & 26.1 & 39.7 & 29.1 & 97.3 & 56.4 \\
5.00 & 26.1 & 40.4 & 29.0 & 95.0 & 55.6 \\
5.25 & 26.1 & 40.7 & 29.0 & 93.1 & 54.9 \\
5.50 & 26.1 & 40.9 & 29.0 & 91.6 & 53.5 \\
5.75 & 26.1 & 41.2 & 29.0 & 89.9 & 52.3 \\
6.00 & 26.1 & 41.6 & 28.9 & 87.9 & 50.6 \\
6.25 & 26.1 & 41.8 & 28.9 & 85.9 & 49.1 \\
6.50 & 26.1 & 42.1 & 28.9 & 83.7 & 47.5 \\
6.75 & 26.0 & 42.4 & 28.9 & 81.8 & 46.4 \\
7.00 & 26.0 & 42.7 & 28.8 & 80.0 & 45.1 \\
7.25 & 26.0 & 42.8 & 28.8 & 78.3 & 44.2 \\
7.50 & 26.0 & 43.1 & 28.8 & 76.6 & 43.0 \\
7.75 & 26.0 & 43.0 & 28.8 & 75.0 & 41.8 \\
8.00 & 26.0 & 42.9 & 28.7 & 72.9 & 40.7 \\
8.25 & 26.0 & 43.0 & 28.7 & 71.5 & 40.3 \\
8.50 & 26.0 & 43.0 & 28.7 & 70.2 & 40.4 \\
8.75 & 26.0 & 43.2 & 28.7 & 68.8 & 39.4 \\
9.00 & 25.9 & 43.0 & 28.7 & 67.4 & 39.2 \\
9.25 & 25.9 & 42.7 & 28.7 & 66.0 & 38.4 \\
9.50 & 25.9 & 42.9 & 28.6 & 64.7 & 37.6 \\
9.75 & 25.9 & 43.0 & 28.6 & 63.4 & 36.9 \\
10.00 & 25.9 & 43.0 & 28.6 & 62.1 & 36.3 \\
10.25 & 25.9 & 43.1 & 28.6 & 60.8 & 35.7 \\
10.50 & 25.9 & 43.2 & 28.6 & 59.7 & 35.1 \\
10.75 & 25.9 & 43.0 & 28.6 & 58.5 & 34.8 \\
11.00 & 25.8 & 42.9 & 28.6 & 57.3 & 34.5 \\
11.25 & 25.8 & 42.8 & 28.5 & 56.3 & 33.9 \\
11.50 & 25.8 & 42.8 & 28.5 & 55.2 & 33.7 \\
11.75 & 25.8 & 42.9 & 28.5 & 54.3 & 33.3 \\
12.00 & 25.8 & 42.8 & 28.5 & 53.3 & 32.8 \\
12.25 & 25.8 & 42.5 & 28.5 & 52.3 & 32.6 \\
12.50 & 25.8 & 42.4 & 28.5 & 51.5 & 32.2 \\
12.75 & 25.8 & 42.3 & 28.5 & 50.7 & 31.7 \\
\hline aata from Tests 1, 4, 6, and 9 are unavailable. &
\end{tabular}




\subsection{Thickness measurements of frozen salt by location}

The thickness of the salt that pooled at the end of the substrate and the thickness of the crust that formed on the substrate upstream from the pooled salt were measured for some tests and the mean and standard deviation of three measurements are provided in Table 14.

Table 14: Thickness of frozen salt crust and pool after spreading $(\mathrm{mm})^{\mathrm{a}}$

\begin{tabular}{ccc}
\hline Test & Crust & Pool \\
\hline 1 & $1^{\mathrm{b}}$ & $3.0 \pm 0.2$ \\
4 & $1.8 \pm 0.3$ & $5.1 \pm 0.1$ \\
6 & $2.5 \pm 1.3$ & $4.4 \pm 0.3$ \\
9 & $2.0 \pm 0.6$ & $4.3 \pm 0.3$ \\
\hline
\end{tabular}

${ }^{\mathrm{a}}$ The provided values are the mean \pm one standard deviation of three measurements.

${ }^{\mathrm{b}}$ Only one measurement was taken.

\subsection{Glovebox conditions}

The measured temperature, $\mathrm{O}_{2}$ content, and $\mathrm{H}_{2} \mathrm{O}$ content of the atmosphere of the argon atmosphere glovebox recorded before and after each spreading test are provided in Table 15.

Table 15: The $\mathrm{O}_{2}$ content, $\mathrm{H}_{2} \mathrm{O}$ content, and temperature of the glovebox atmosphere measured before and after each test

\begin{tabular}{cccccccc}
\hline & \multicolumn{2}{c}{$\mathrm{O}_{2}$ content $(\mathrm{ppm})$} & \multicolumn{2}{c}{$\mathrm{H}_{2} \mathrm{O}$ content $(\mathrm{ppm})$} & \multicolumn{3}{c}{ Temp. $\left({ }^{\circ} \mathrm{C}\right)$ of atmosphere } \\
\cline { 2 - 7 } Test & Before & After & Before & After & Before & After & Max. \\
\hline 1 & 3 & 3 & $<1$ & $<1$ & 27 & 27 & 27 \\
2 & $<1$ & $<1$ & $<1$ & $<1$ & 26 & 26 & 26 \\
3 & $<1$ & $<1$ & $<1$ & $<1$ & 28 & 26 & 28 \\
4 & 5 & 5 & $<1$ & $<1$ & 26 & 26 & 27 \\
5 & $<1$ & $<1$ & $<1$ & $<1$ & 28 & 29 & 29 \\
6 & 2 & 2 & $<1$ & $<1$ & 28 & 27 & 28 \\
7 & $<1$ & $<1$ & $<1$ & $<1$ & 27 & 27 & 28 \\
8 & $<1$ & $<1$ & $<1$ & $<1$ & 30 & 28 & 30 \\
9 & 2 & 2 & $<1$ & $<1$ & 28 & 28 & 29 \\
\hline
\end{tabular}




\subsection{Composition of frozen salt}

The concentrations of the major cations and the trace metals in the source batch of FLiNaK used for these tests reported by Lichtenstein et al., 2020 are reproduced in Table 16 and Table 17, respectively. The major cation concentrations were determined by using ICP-OES and the trace metal concentrations were determined by using ICP-MS (Lichtenstein et al., 2020). Contamination from the ceramic mortar and pestle used to grind the FLiNaK into a powder is likely responsible for the high calcium concentration reported in Table 17. That contamination may affect the salt property values slightly but does not affect insights drawn from the studies described herein.

Table 16: Concentrations of major components in FLiNaK before the tests (mol \%)

\begin{tabular}{cccc}
\hline Sample & LiF & NaF & KF \\
\hline 1 & 47.0 & 11.6 & 41.4 \\
2 & 46.3 & 11.6 & 42.1 \\
3 & 46.1 & 11.3 & 42.6 \\
\hline Mean $\pm 1 \sigma$ & $46.5 \pm 0.5$ & $11.5 \pm 0.2$ & $42.0 \pm 0.6$ \\
\hline
\end{tabular}

Table 17: Concentrations of trace metals in the salt before the tests $(\mathrm{ppm})^{\mathrm{a}}$

\begin{tabular}{cccccccc}
\hline Sample & $\mathrm{Ca}$ & $\mathrm{Cr}$ & $\mathrm{Mn}$ & $\mathrm{Fe}$ & $\mathrm{Ni}$ & $\mathrm{Rb}$ & $\mathrm{Cs}$ \\
\hline 1 & 1490 & $<11$ & $<4$ & $<200$ & 115 & 31.9 & 2.3 \\
2 & 944 & $<8$ & $<2$ & $<140$ & 137 & 25.8 & 1.8 \\
3 & 510 & $<7$ & $<3$ & $<120$ & 118 & 26.3 & 1.4 \\
\hline Mean $\pm 1 \sigma$ & $980 \pm 490$ & - & - & - & $123 \pm 12$ & $28 \pm 3$ & $1.8 \pm 0.4$ \\
\hline
\end{tabular}

${ }^{a}$ The number after $<$ is the detection limit.

The salt that froze on the stainless steel sheet was analyzed for major cation (lithium, sodium, and potassium) and trace metal (calcium, manganese, iron, nickel, chromium, cesium, and rubidium) concentrations by using ICP-MS following the methods reported in Section 2.3 in Thomas and Jackson, 2021. Frozen salt samples were taken from different locations on the stainless steel substrate to compare compositions within the flow path. Table 18 and Table 19 provide the concentrations of major components and trace metals, respectively, that were measured in the salt that was recovered after spreading for each test. 
Table 18: Composition of frozen salt samples collected from spreading tests

\begin{tabular}{|c|c|c|c|c|c|c|c|}
\hline Test & $\begin{array}{c}\text { CsF \& Csl } \\
\text { in salt? }\end{array}$ & $\begin{array}{c}\text { Sample } \\
\text { type }\end{array}$ & $\begin{array}{c}\mathrm{LiF} \\
(\mathrm{mol} \%)\end{array}$ & $\begin{array}{c}\mathrm{NaF} \\
(\mathrm{mol} \%)\end{array}$ & $\begin{array}{c}\mathrm{KF} \\
(\mathrm{mol} \%)\end{array}$ & $\begin{array}{c}\mathrm{CsF} \\
(\mathrm{mol} \%)\end{array}$ & $\begin{array}{c}\mathrm{Csl}^{\mathrm{a}} \\
(\mathrm{mol} \%)\end{array}$ \\
\hline \multirow{2}{*}{1} & \multirow{2}{*}{ No } & Crust & 47.7 & 11.1 & 41.2 & - & - \\
\hline & & Pool & 46.7 & 11.0 & 42.3 & - & - \\
\hline \multirow{2}{*}{2} & \multirow{2}{*}{ No } & Crust & 47.3 & 11.2 & 41.5 & - & - \\
\hline & & Pool & 47.5 & 11.4 & 41.2 & - & - \\
\hline \multirow{2}{*}{3} & \multirow{2}{*}{ No } & Pool 1 & 47.5 & 11.3 & 41.2 & - & - \\
\hline & & Pool 2 & 47.4 & 11.4 & 41.2 & - & - \\
\hline \multirow{2}{*}{4} & \multirow{2}{*}{ Yes } & Crust & 46.3 & 11.0 & 41.8 & 0.82 & 0.04 \\
\hline & & Pool & 46.9 & 11.1 & 41.2 & 0.80 & 0.06 \\
\hline \multirow{2}{*}{5} & \multirow{2}{*}{ No } & Crust & 47.4 & 11.4 & 41.2 & - & - \\
\hline & & Pool & - & - & - & - & - \\
\hline \multirow{2}{*}{6} & \multirow{2}{*}{ No } & Crust & 47.2 & 11.1 & 41.7 & - & - \\
\hline & & Pool & 47.6 & 11.1 & 41.3 & - & - \\
\hline \multirow{2}{*}{7} & \multirow{2}{*}{ No } & Crust & - & - & - & - & - \\
\hline & & Pool & 47.3 & 11.7 & 41.0 & - & - \\
\hline \multirow{2}{*}{8} & \multirow{2}{*}{ No } & Crust & - & - & - & - & - \\
\hline & & Pool & 47.6 & 11.5 & 41.0 & - & - \\
\hline \multirow{2}{*}{9} & \multirow{2}{*}{ No } & Crust & 47.4 & 11.3 & 41.3 & - & - \\
\hline & & Pool & 47.6 & 11.3 & 41.1 & - & - \\
\hline \multicolumn{3}{|c|}{ Crust mean $( \pm 1 \sigma)^{b}$} & $47.4 \pm 0.2$ & $11.3 \pm 0.1$ & $41.3 \pm 0.2$ & - & - \\
\hline \multicolumn{3}{|c|}{ Pool mean $( \pm 1 \sigma)^{b}$} & $47.4 \pm 0.3$ & $11.3 \pm 0.3$ & $41.3 \pm 0.5$ & - & - \\
\hline
\end{tabular}

${ }^{a} \mathrm{Mol} \%$ of Csl was calculated assuming all detected iodine was present as Csl.

$\mathrm{b}$ The results from Test 4 were excluded from the mean. 
Table 19: Trace metal composition of frozen salt samples collected from spreading tests ${ }^{a, b}$

\begin{tabular}{|c|c|c|c|c|c|c|c|c|c|}
\hline Test & $\begin{array}{c}\text { CsF \& Csl } \\
\text { in salt? }\end{array}$ & $\begin{array}{c}\text { Sample } \\
\text { type }\end{array}$ & $\begin{array}{c}\mathrm{Ca} \\
(\mathrm{ppm})\end{array}$ & $\begin{array}{c}\mathrm{Cr} \\
(\mathrm{ppm})\end{array}$ & $\begin{array}{c}\mathrm{Mn} \\
(\mathrm{ppm})\end{array}$ & $\begin{array}{c}\text { Fe } \\
(\mathrm{ppm})\end{array}$ & $\begin{array}{c}\mathrm{Ni} \\
(\mathrm{ppm})\end{array}$ & $\begin{array}{c}\mathrm{Rb} \\
(\mathrm{ppm})\end{array}$ & $\begin{array}{c}\mathrm{Cs}^{\mathrm{c}} \\
(\mathrm{ppm})\end{array}$ \\
\hline \multirow{2}{*}{1} & \multirow{2}{*}{ No } & Crust & 1000 & $<1.5$ & $<2.2$ & $<38.9$ & 5.8 & 22.5 & 10.7 \\
\hline & & Pool & 616 & $<1.3$ & $<2.6$ & $<34.9$ & 23.2 & 22.4 & 2.2 \\
\hline \multirow{2}{*}{2} & \multirow{2}{*}{ No } & Crust & 359 & $<2.6$ & 1.5 & 13.7 & 26.3 & 24.2 & 1.4 \\
\hline & & Pool & 344 & $<1.3$ & 1.8 & 14.3 & 8.9 & 23.5 & 1.5 \\
\hline \multirow{2}{*}{3} & \multirow{2}{*}{ No } & Crust & 204 & $<1.7$ & 4.1 & 8.9 & 16.5 & 23.4 & 1.5 \\
\hline & & Pool & 1370 & $<1.7$ & 3.9 & 25.7 & 7.6 & 24.0 & 1.5 \\
\hline \multirow{2}{*}{4} & \multirow{2}{*}{ Yes } & Crust & $<181$ & $<1.5$ & 2.7 & $<46.0$ & 20.3 & 21.8 & - \\
\hline & & Pool & $<210$ & $<1.5$ & 2.8 & $<45.9$ & 43.3 & 21.8 & - \\
\hline \multirow{2}{*}{5} & \multirow{2}{*}{ No } & Crust & 932 & $<1.3$ & 6.2 & 12.9 & 2.6 & 23.9 & 1.5 \\
\hline & & Pool & - & - & - & - & - & - & - \\
\hline \multirow{2}{*}{6} & \multirow{2}{*}{ No } & Crust & 955 & $<1.9$ & 4.7 & $<51.7$ & 8.1 & 21.8 & 22.6 \\
\hline & & Pool & 392 & $<1.1$ & 3.4 & $<29.8$ & 17.8 & 21.5 & 2.1 \\
\hline \multirow{2}{*}{7} & \multirow{2}{*}{ No } & Crust & - & - & - & - & - & - & - \\
\hline & & Pool & 581 & $<1.3$ & 8.1 & 6.47 & $<0.5$ & 23.8 & 1.4 \\
\hline \multirow{2}{*}{8} & \multirow{2}{*}{ No } & Crust & - & - & - & - & - & - & - \\
\hline & & Pool & 338 & $<1.5$ & 6.0 & 23.1 & 3.8 & 22.7 & 1.3 \\
\hline \multirow{2}{*}{9} & \multirow{2}{*}{ No } & Crust & 912 & $<1.8$ & 5.0 & $<42.1$ & 10.6 & 21.2 & 5.4 \\
\hline & & Pool & 608 & $<1.1$ & 4.4 & $<28.2$ & 7.2 & 21.4 & 4.5 \\
\hline \multicolumn{3}{|c|}{ Crust mean $( \pm 1 \sigma)^{\mathrm{c}}$} & $730 \pm 350$ & - & $4 \pm 2$ & - & $13 \pm 9$ & $23 \pm 1$ & $7 \pm 8$ \\
\hline \multicolumn{3}{|c|}{ Pool mean $( \pm 1 \sigma)^{\mathrm{c}}$} & $610 \pm 360$ & - & $4 \pm 2$ & - & $16 \pm 14$ & $23 \pm 1$ & $2 \pm 1$ \\
\hline
\end{tabular}

a The number after $<$ is the detection limit.

b The Cs concentration of FLiNaK doped with CsF and CsI (Test 4) is reported in Table 18.

c The results from Test 4 were excluded from the mean.

\subsection{Composition of condensation on platinum foil}

Condensed vapor from the pool of molten salt containing cesium and iodine as surrogate fission products that formed at the end of the stainless steel sheet was collected on an acid-washed platinum foil $\left(7.5 \mathrm{~cm}^{2}\right)$. The foil was placed above the location where the salt pools and was supported by the wall of the stainless steel sheet and a stainless steel rod on two sides (Figure 3). A control foil that was exposed to the glovebox atmosphere for approximately one hour was also tested. After the test, the platinum foil was rinsed with water and the water was analyzed for lithium, sodium, potassium, cesium, and iodine by using ICP-MS. The results from the water rinse are provided in Table 20.

A subsequent acid leach was performed on the platinum foil and the leachate was analyzed for lithium, sodium, potassium, and cesium by using ICP-MS (Table 20). Little additional lithium, sodium, potassium, or cesium was recovered by the acid leach, which indicates a water leach is sufficient for quantifying condensate that forms on a platinum foil. 


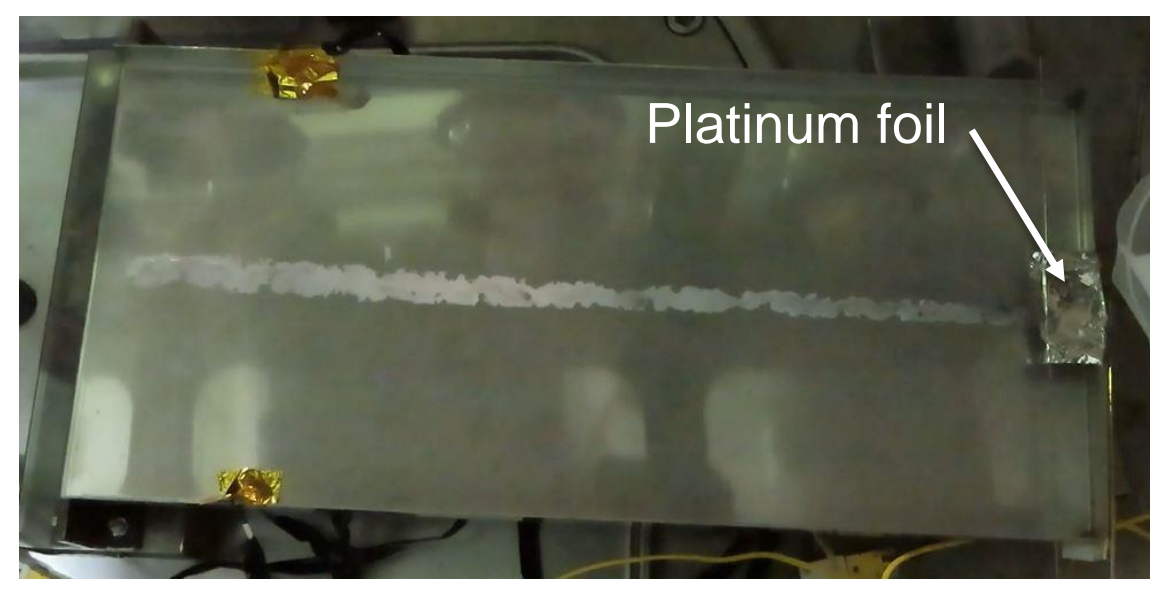

Figure 3: Image of stainless steel sheet that shows the position of the platinum foil for condensation collection.

Table 20: Composition of condensate collected on platinum foil above salt pool in Test 4

\begin{tabular}{ccccccc}
\hline Test & Method $^{\mathrm{b}}$ & $\begin{array}{c}\mathrm{Li} \\
(\mu \mathrm{g})\end{array}$ & $\begin{array}{c}\mathrm{Na} \\
(\mu \mathrm{g})\end{array}$ & $\begin{array}{c}\mathrm{K} \\
(\mu \mathrm{g})\end{array}$ & $\begin{array}{c}\text { Cs } \\
(\mu \mathrm{g})\end{array}$ & $\begin{array}{c}\mathrm{I} \\
(\mu \mathrm{g})\end{array}$ \\
\hline \multirow{3}{*}{ Control $^{\mathrm{c}}$} & Water & 0.31 & 5.29 & $<7.69$ & 0.08 & $<0.25$ \\
& Acid & 0.08 & 0.36 & $<3.91$ & 0.26 & - \\
\cline { 2 - 7 } & Combined & 0.39 & 5.65 & $\mathrm{BDL}$ & 0.34 & - \\
\cline { 2 - 7 } Sample & Water & 1.09 & 11.00 & 12.6 & 1.61 & $<0.31$ \\
& Acid & 0.15 & 0.19 & $<3.91$ & 0.09 & - \\
\cline { 2 - 7 } & Combined & 1.24 & 11.19 & 12.6 & 1.70 & - \\
\hline
\end{tabular}

${ }^{a}$ The number following the $<$ is the detection limit.

$\mathrm{b}$ The foils were first leached with water and then leached a second time with acid. The composition in both the water and acid leachate is provided.

c The control foil was exposed to the glovebox atmosphere for approximately one hour. 


\section{Molten salt flowing and freezing behavior through tubing}

The data provided in this section correspond to Section 4 in Thomas and Jackson, 2021, and the experimental design and methods are described therein. Table 21 provides a summary of the conditions of each test conducted to study FLiNaK flowing and freezing in stainless steel tubing.

Table 21: Summary of test conditions to study molten FLiNaK flowing through tubing ${ }^{\text {a }}$

\begin{tabular}{ccccc}
\hline Test & $\begin{array}{c}\text { Target initial salt } \\
\text { temp. }\left({ }^{\circ} \mathrm{C}\right)^{\mathrm{b}}\end{array}$ & $\begin{array}{c}\text { Max salt temp. } \\
\left({ }^{\circ} \mathrm{C}\right)^{\mathrm{c}}\end{array}$ & $\begin{array}{c}\text { Avg. pour rate } \\
\left(\mathrm{g} \mathrm{s}^{-1}\right)\end{array}$ & $\begin{array}{c}\text { Tube inner } \\
\text { diameter (in.) }\end{array}$ \\
\hline 1 & 675 & 660 & 14.8 & $1 / 4$ \\
2 & 675 & 660 & 16.4 & $3 / 16$ \\
3 & 525 & 490 & 18.3 & $3 / 16$ \\
4 & 800 & 775 & 10.7 & $3 / 16$ \\
\hline
\end{tabular}

${ }^{\text {a } A l l ~ t e s t s ~ w e r e ~ p e r f o r m e d ~ w i t h ~ a ~ p o u r ~ m a s s ~ o f ~} 25 \mathrm{~g}$.

${ }^{\mathrm{b}}$ The actual salt temperatures may have slightly differed from the target temperature.

c The maximum temperature of the salt measured by the IR camera (uncorrected for emissivity) provided an estimate of the actual salt temperature as it was poured.

\subsection{Visible video from tests of molten salt flowing on stainless steel funnel cone}

Visible video of the molten salt flowing on the stainless steel funnel cone and draining into the stainless steel tubing was filmed using a visible camera mounted on a tripod and is available upon reasonable request. A list of available videos is provided in Table 22.

Table 22: List of available visible video of flowing tests

\begin{tabular}{cc}
\hline Test & Filename \\
\hline 1 & FunnelVideo_Test1.mp4 \\
2 & FunnelVideo_Test2.mp4 \\
3 & FunnelVideo_Test3.mp4 \\
4 & FunnelVideo_Test4.mp4 \\
\hline
\end{tabular}

\subsection{Temperature measurement of the stainless steel tubing}

Frames from the processed IR video of the outer tube temperature were exported to .csv files at a rate of five frames per second and details on the available frames is provided in Table 23 . The data processing technique to correct for the emissivity of the outer tube surface is described in Section 4.1.2.3 of Thomas and Jackson, 2021. The video frames were cropped to only include the region of the camera field of view that shows the funnel tube. Some background is also included in the field of view, but the background is distinguished from the tube by its much lower temperature in comparison with the outer tube surface temperature. It should be noted that the funnel tube may have moved slightly throughout the test duration and careful attention should be 
paid to the position of the tube. A time stamp file is also available for each test to provide the time that the frame was collected after the salt was poured.

Table 23: Information on exported IR video frames from flowing tests

\begin{tabular}{ccc}
\hline Test & Number of frames & Total duration $(\mathrm{s})$ \\
\hline 1 & 790 & 131.5 \\
2 & 532 & 88.5 \\
3 & 637 & 106.0 \\
4 & 648 & 107.8 \\
\hline
\end{tabular}

\subsection{Temperature of the underside of the stainless steel beaker}

The temperature of the underside of the stainless steel beaker that collected the molten salt that passed through the funnel tube was recorded with thermocouples attached to the underside surface and the results for each test are provided in Table 24. A description of the temperature measurement method and its performance is presented in Appendix B of Thomas and Jackson, 2021.

Table 24: Temp. $\left({ }^{\circ} \mathrm{C}\right)$ measured by thermocouple attached to underside of stainless steel beaker

\begin{tabular}{ccccc}
\hline \multirow{2}{*}{ Time (min.) } & 1 & 2 & 3 & 4 \\
\hline 0.00 & 29.4 & 29.1 & 27.7 & 29.7 \\
0.25 & 288.9 & 256.5 & 191.2 & 226.1 \\
0.50 & 352.1 & 317.3 & 258.0 & 282.6 \\
0.75 & 384.5 & 350.1 & 291.8 & 314.4 \\
1.00 & 402.1 & 369.3 & 311.1 & 335.5 \\
1.25 & 412.4 & 379.9 & 322.4 & 350.1 \\
1.50 & 418.1 & 386.4 & 328.1 & 360.1 \\
1.75 & 421.0 & 390.2 & 329.9 & 367.2 \\
2.00 & 421.4 & 392.4 & 328.5 & 372.1 \\
2.25 & 419.1 & 392.4 & 324.2 & 375.3 \\
2.50 & 414.1 & 391.1 & 317.2 & 377.3 \\
2.75 & 405.0 & 389.9 & 308.8 & 378.0 \\
3.00 & 392.7 & 386.8 & 299.6 & 375.5 \\
3.25 & 379.2 & 380.2 & 290.3 & 368.6 \\
3.50 & 365.6 & 369.7 & 281.2 & 358.9 \\
3.75 & 352.2 & 357.6 & 272.3 & 348.5 \\
4.00 & 339.6 & 345.4 & 263.8 & 337.8 \\
\hline
\end{tabular}


Table 24: (cont.)

\begin{tabular}{|c|c|c|c|c|}
\hline \multirow{2}{*}{ Time (min.) } & \multicolumn{4}{|c|}{ Test } \\
\hline & 1 & 2 & 3 & 4 \\
\hline 4.25 & 327.7 & 333.5 & 255.5 & 327.1 \\
\hline 4.50 & 316.3 & 322.0 & 247.5 & 316.7 \\
\hline 4.75 & 305.5 & 310.9 & 239.9 & 306.5 \\
\hline 5.00 & 295.2 & 300.5 & 232.6 & 296.8 \\
\hline 5.25 & 285.3 & 290.5 & 225.5 & 287.6 \\
\hline 5.50 & 276.0 & 281.0 & 218.8 & 278.7 \\
\hline 5.75 & 267.1 & 271.9 & 212.3 & 270.0 \\
\hline 6.00 & 258.7 & 263.2 & 206.2 & 261.8 \\
\hline 6.25 & 250.6 & 254.8 & 200.3 & 253.9 \\
\hline 6.50 & 242.8 & 246.9 & 194.7 & 246.2 \\
\hline 6.75 & 235.5 & 239.3 & 189.2 & 239.0 \\
\hline 7.00 & 228.5 & 232.1 & 184.0 & 232.0 \\
\hline 7.25 & 221.9 & 225.1 & 179.1 & 225.3 \\
\hline 7.50 & 215.5 & 218.5 & 174.3 & 218.9 \\
\hline 7.75 & 209.3 & 212.2 & 169.7 & 212.7 \\
\hline 8.00 & 203.5 & 206.1 & 165.4 & 206.8 \\
\hline 8.25 & 197.9 & 200.2 & 161.2 & 201.2 \\
\hline 8.50 & 192.5 & 194.6 & 157.1 & 195.8 \\
\hline 8.75 & 187.4 & 189.3 & 153.3 & 190.6 \\
\hline 9.00 & 182.5 & 184.2 & 149.6 & 185.7 \\
\hline 9.25 & 177.8 & 179.4 & 146.0 & 180.9 \\
\hline 9.50 & 173.4 & 174.7 & 142.5 & 176.3 \\
\hline 9.75 & 169.0 & 170.2 & 139.2 & 171.9 \\
\hline 10.00 & 164.8 & 165.9 & 135.9 & 167.7 \\
\hline 10.25 & 160.9 & 161.8 & 132.8 & 163.6 \\
\hline 10.50 & 157.0 & 157.8 & 129.8 & 159.7 \\
\hline 10.75 & 153.3 & 154.0 & 126.9 & 155.9 \\
\hline 11.00 & 149.7 & 150.4 & 124.0 & 152.3 \\
\hline 11.25 & 146.3 & 146.8 & 121.3 & 148.8 \\
\hline 11.50 & 143.0 & 143.4 & 118.7 & 145.4 \\
\hline 11.75 & 139.7 & 140.1 & 116.2 & 142.1 \\
\hline 12.00 & 136.7 & 137.0 & 113.7 & 138.9 \\
\hline 12.25 & 133.7 & 133.9 & 111.4 & 135.9 \\
\hline 12.50 & 130.8 & 130.9 & 109.1 & 132.9 \\
\hline 12.75 & 128.0 & 128.0 & 106.9 & 130.1 \\
\hline 13.00 & 125.3 & 125.3 & 104.8 & 127.3 \\
\hline 13.25 & 122.6 & 122.6 & 102.7 & 124.6 \\
\hline 13.50 & 120.1 & 120.0 & 100.7 & 122.0 \\
\hline 13.75 & 117.6 & 117.5 & 98.8 & 119.5 \\
\hline 14.00 & 115.3 & 115.1 & 96.9 & 117.1 \\
\hline
\end{tabular}


Table 24: (cont.)

\begin{tabular}{|c|c|c|c|c|}
\hline \multirow{2}{*}{ Time (min.) } & \multicolumn{4}{|c|}{ Test } \\
\hline & 1 & 2 & 3 & 4 \\
\hline 14.00 & 115.3 & 115.1 & 96.9 & 117.1 \\
\hline 14.25 & 113.0 & 112.7 & 95.1 & 114.7 \\
\hline 14.50 & 110.8 & 110.5 & 93.3 & 112.5 \\
\hline 14.75 & 108.6 & 108.2 & 91.6 & 110.3 \\
\hline 15.00 & 106.5 & 106.1 & 90.0 & 108.2 \\
\hline 15.25 & 104.5 & 104.0 & 88.4 & 106.1 \\
\hline 15.50 & 102.5 & 102.0 & 86.8 & 104.1 \\
\hline 15.75 & 100.6 & 100.1 & 85.3 & 102.1 \\
\hline 16.00 & 98.8 & 98.2 & 83.8 & 100.3 \\
\hline 16.25 & 97.0 & 96.4 & 82.4 & 98.5 \\
\hline 16.50 & 95.3 & 94.6 & 81.0 & 96.7 \\
\hline 16.75 & 93.6 & 92.9 & 79.7 & 95.0 \\
\hline 17.00 & 92.0 & 91.3 & 78.4 & 93.3 \\
\hline 17.25 & 90.4 & 89.7 & 77.1 & 91.7 \\
\hline 17.50 & 88.8 & 88.1 & 75.9 & 90.2 \\
\hline 17.75 & 87.3 & 86.6 & 74.7 & 88.6 \\
\hline 18.00 & 85.9 & 85.1 & 73.5 & 87.1 \\
\hline 18.25 & 84.5 & 83.7 & 72.3 & 85.7 \\
\hline 18.50 & 83.1 & 82.3 & 71.2 & 84.3 \\
\hline 18.75 & 81.8 & 81.0 & 70.1 & 82.9 \\
\hline 19.00 & 80.5 & 79.6 & 69.1 & 81.6 \\
\hline 19.25 & 79.2 & 78.4 & 68.1 & 80.3 \\
\hline 19.50 & 78.0 & 77.1 & 67.1 & 79.0 \\
\hline 19.75 & 76.8 & 75.9 & 66.2 & 77.8 \\
\hline 20.00 & 75.7 & 74.7 & 65.2 & 76.7 \\
\hline 20.25 & 74.5 & 73.5 & 64.3 & 75.5 \\
\hline 20.50 & 73.4 & 72.4 & 63.5 & 74.4 \\
\hline 20.75 & 72.4 & 71.3 & 62.6 & 73.4 \\
\hline 21.00 & 71.4 & 70.2 & 61.8 & 72.3 \\
\hline 21.25 & 70.4 & 69.2 & 60.9 & 71.3 \\
\hline 21.50 & 69.4 & 68.2 & 60.2 & 70.3 \\
\hline 21.75 & 68.4 & 67.2 & 59.4 & 69.4 \\
\hline 22.00 & 67.5 & 66.2 & 58.7 & 68.5 \\
\hline 22.25 & 66.6 & 65.3 & 57.9 & 67.6 \\
\hline 22.50 & 65.7 & 64.4 & 57.3 & 66.7 \\
\hline 22.75 & 64.8 & 63.5 & 56.6 & 65.8 \\
\hline 23.00 & 64.0 & 62.7 & 56.0 & 65.0 \\
\hline 23.25 & 63.2 & 61.8 & 55.3 & 64.2 \\
\hline 23.50 & 62.4 & 61.0 & 54.7 & 63.4 \\
\hline 23.75 & 61.6 & 60.2 & 54.1 & 62.6 \\
\hline
\end{tabular}


Table 24: (cont.)

\begin{tabular}{ccccc}
\hline \multirow{2}{*}{ Time (min.) } & \multicolumn{4}{c}{ Test } \\
& 1 & 2 & 3 & 4 \\
\hline 24.00 & 60.8 & 59.5 & 53.5 & 61.9 \\
24.25 & 60.1 & 58.7 & 52.9 & 61.1 \\
24.50 & 59.4 & 58.0 & 52.3 & 60.4 \\
24.75 & 58.7 & 57.3 & 51.8 & 59.7 \\
25.00 & 58.0 & 56.6 & 51.3 & 59.0 \\
25.25 & 57.4 & 55.9 & 50.8 & 58.4 \\
25.50 & 56.7 & 55.3 & 50.2 & 57.7 \\
25.75 & 56.1 & 54.6 & 49.7 & 57.1 \\
26.00 & 55.5 & 54.0 & 49.2 & 56.5 \\
26.25 & 54.9 & 53.5 & 48.7 & 55.9 \\
26.50 & 54.4 & 52.9 & 48.2 & 55.3 \\
26.75 & 53.8 & 52.3 & 47.7 & 54.7 \\
27.00 & 53.3 & 51.8 & 47.2 & 54.2 \\
27.25 & 52.7 & 51.3 & 46.7 & 53.6 \\
27.50 & 52.2 & 50.7 & 46.2 & 53.1 \\
27.75 & 51.7 & 50.2 & 45.8 & 52.6 \\
28.00 & 51.2 & 49.7 & 45.4 & 52.1 \\
28.25 & 50.8 & 49.2 & 45.0 & 51.6 \\
28.50 & 50.0 & 48.7 & 44.7 & 51.1 \\
28.75 & 48.9 & 48.3 & 44.3 & 50.7 \\
29.00 & 47.9 & 47.9 & 43.9 & 50.2 \\
29.25 & 46.9 & 47.4 & 43.5 & 49.7 \\
29.50 & 46.0 & 47.0 & 43.1 & 49.2 \\
29.75 & 45.2 & 46.6 & 42.8 & 48.7 \\
30.00 & 44.4 & 46.2 & 42.4 & 48.3 \\
\hline & & & &
\end{tabular}

The maximum temperature recorded at the beaker underside and the average thickness of the frozen salt pool in the beaker after it had cooled to room temperature are summarized in Table 25.

Table 25: The maximum temperature recorded at the beaker underside, the thickness of the frozen salt pool, and salt mass in beaker ${ }^{\mathrm{a}}$

\begin{tabular}{cccc}
\hline Test & $\begin{array}{c}\text { Max. beaker underside } \\
\text { temp. }\left({ }^{\circ} \mathrm{C}\right)\end{array}$ & $\begin{array}{c}\text { Salt thickness } \\
(\mathrm{mm})^{\mathrm{b}}\end{array}$ & $\begin{array}{c}\text { Salt mass in } \\
\text { beaker }(\mathrm{g})\end{array}$ \\
\hline 1 & 422 & $4.5 \pm 0.6$ & 21.6 \\
2 & 393 & $5.6 \pm 0.3$ & 17.9 \\
3 & 330 & $5.3 \pm 0.2$ & 17.1 \\
4 & 378 & $5.0 \pm 0.1$ & 16.8 \\
\hline
\end{tabular}

${ }^{a}$ The conditions of each test are provided in Table 21.

${ }^{\mathrm{b}}$ Mean \pm one standard deviation of three measurements. 


\subsection{Flow rate of salt through tubing}

The flow rate of the salt through the tubing was determined by using a data logging scale that recorded the mass of the beaker at one-second intervals. The scale started to record once it detected a change from the tare weight. Table 26 reports the mass of salt in the stainless steel beaker as a function of time after the scale first detected a weight for the four tests in this section.

Table 26: The mass $(\mathrm{g})$ of salt recorded in stainless steel beaker as a function of time

\begin{tabular}{ccccc}
\hline & \multicolumn{4}{c}{ Test } \\
Time $(\mathrm{s})$ & 1 & 2 & 3 & 4 \\
\hline 0 & 2.0 & 10.1 & 5.8 & 5.7 \\
1 & 14.2 & 18.1 & 10.9 & 15.7 \\
2 & 20.4 & 18.2 & 14.4 & 16.8 \\
3 & 20.9 & 18.1 & 15.3 & 17.0 \\
4 & 21.1 & 18.1 & 15.9 & 17.0 \\
5 & 21.0 & 18.1 & 16.2 & 17.0 \\
6 & 21.0 & 18.1 & 16.4 & 16.9 \\
7 & 20.9 & 18.1 & 16.5 & 16.9 \\
8 & 20.9 & 18.1 & 16.5 & 16.9 \\
9 & 20.9 & 18.1 & 16.5 & 16.9 \\
10 & 20.9 & 18.1 & 16.5 & 16.9 \\
11 & 20.9 & 18.1 & 16.5 & 16.9 \\
12 & 20.9 & 18.1 & 16.5 & 16.9 \\
13 & 20.9 & 18.1 & 16.5 & 16.9 \\
14 & 20.9 & 18.0 & 16.5 & 16.9 \\
15 & 20.9 & 18.0 & 16.5 & 16.9 \\
16 & 20.9 & 18.0 & 16.5 & 16.9 \\
17 & 20.9 & 18.0 & 16.5 & 16.9 \\
18 & 20.9 & 18.0 & 16.5 & 16.9 \\
19 & 21.0 & 18.0 & 16.5 & 16.8 \\
20 & 21.0 & 18.0 & 16.5 & 16.8 \\
\hline
\end{tabular}

\subsection{Mass balance analysis}

Table 27 presents the amounts of frozen salt that were recovered from the top of the funnel, from inside the tubing, from the stainless steel beaker below the tubing, and not recovered as a fraction of the total mass poured for the four tests conditions in this section. 
Table 27: Results from mass balance analysis of frozen FLiNaK salt

\begin{tabular}{ccccccc}
\hline Test & $\begin{array}{c}\text { Mass } \\
\text { poured }(\mathrm{g})\end{array}$ & $\begin{array}{c}\text { Mass } \\
\text { recovered } \\
\text { from top }(\mathrm{g})\end{array}$ & $\begin{array}{c}\text { Mass } \\
\text { recovered } \\
\text { from tube }(\mathrm{g})\end{array}$ & $\begin{array}{c}\text { Mass } \\
\text { recovered from } \\
\text { beaker }(\mathrm{g})\end{array}$ & $\begin{array}{c}\text { Total mass } \\
\text { recovered }(\mathrm{g})\end{array}$ & $\begin{array}{c}\text { Mass } \\
\text { lost }(\mathrm{g})\end{array}$ \\
\hline 1 & 24.7 & 1.8 & 0.8 & 21.6 & 24.3 & 0.4 \\
2 & 24.5 & 2.1 & 3.6 & 17.9 & 23.6 & 1.0 \\
3 & 23.3 & 4.2 & 1.6 & 17.1 & 22.9 & 0.4 \\
4 & 24.3 & 2.1 & 4.2 & 16.8 & 23.1 & 1.2 \\
\hline
\end{tabular}

\subsection{Glovebox conditions}

The measured temperature, $\mathrm{O}_{2}$ content, and $\mathrm{H}_{2} \mathrm{O}$ content of the atmosphere of the argon atmosphere glovebox recorded before and after each test are provided in Table 28.

Table 28: The $\mathrm{O}_{2}$ content, $\mathrm{H}_{2} \mathrm{O}$ content, and temperature of the glovebox atmosphere measured before and after each test

\begin{tabular}{cccccccc}
\hline & \multicolumn{2}{c}{$\mathrm{O}_{2}$ content $(\mathrm{ppm})$} & \multicolumn{2}{c}{$\mathrm{H}_{2} \mathrm{O}$ content $(\mathrm{ppm})$} & \multicolumn{3}{c}{ Temp. $\left({ }^{\circ} \mathrm{C}\right)$ of atmosphere } \\
\cline { 2 - 7 } Test & Before & After & Before & After & Before & After & Max. \\
\hline 1 & 2 & 2 & 1 & 1 & 32 & 30 & 34 \\
2 & 1 & 1 & $<1$ & $<1$ & 27 & 26 & 27 \\
3 & 1 & 1 & 2 & 2 & 29 & 29 & 29 \\
4 & 2 & 2 & $<1$ & $<1$ & 29 & 29 & 30 \\
\hline
\end{tabular}

\section{Stainless steel corrosion kinetics in molten salt}

The data provided in this section correspond to Section 5 in Thomas and Jackson, 2021, and the experimental design and methods are described therein. Table 29 provides a summary of the electrochemical corrosion test conditions conducted with 316 stainless steel in molten FLiNaK.

Table 29: Summary of test conditions to study the corrosion of 316 stainless steel in molten FLiNaK

\begin{tabular}{ccccc}
\hline Test & Salt temp. $\left({ }^{\circ} \mathrm{C}\right)$ & PD scan $^{\mathrm{a}}$ & PS test $^{\mathrm{b}}$ & $\begin{array}{c}\text { PS hold potential } \\
(\text { V above EconR })\end{array}$ \\
\hline 1 & 500 & $\mathrm{Y}$ & $\mathrm{Y}$ & 0.2 \\
2 & 650 & $\mathrm{Y}$ & $\mathrm{N}$ & $\mathrm{n} / \mathrm{a}$ \\
3 & 800 & $\mathrm{Y}$ & $\mathrm{Y}$ & 0.1 \\
\hline a PD = Potentiodynamic & & & \\
b PS $=$ Potentiostatic & & &
\end{tabular}


The files of the potentiodynamic (PD) scans of 316 stainless steel coupons in molten FLiNaK at temperatures of $500^{\circ} \mathrm{C}, 650^{\circ} \mathrm{C}$, and $800^{\circ} \mathrm{C}$ are available upon reasonable request. The PD scan consists of the stainless steel surface potential (V vs. $\mathrm{Li} / \mathrm{Li}^{+}$) that was measured as a function of current density $\left(\mathrm{A} \mathrm{cm}^{-2}\right)$. The files of the potentiostatic (PS) tests are available upon reasonable request and consist of the current density $\left(\mathrm{A} \mathrm{cm}^{-2}\right)$ measured as a function of time at fixed temperature and stainless steel surface potential. Table 30 provides a summary of the steady corrosion currents that were measured during PS tests at fixed temperature and surface potential and the mass corrosion rates and penetration rates that were calculated from the steady corrosion currents.

Table 30: Summary of steady corrosion currents measured during PS tests and calculated mass corrosion rates and penetration rates

\begin{tabular}{|c|c|c|c|c|c|c|}
\hline \multirow[b]{2}{*}{ Test } & \multirow[b]{2}{*}{$\begin{array}{l}\text { Salt temp. } \\
\qquad\left({ }^{\circ} \mathrm{C}\right)\end{array}$} & \multicolumn{2}{|c|}{ PS hold potential } & \multirow{2}{*}{$\begin{array}{l}\text { Steady current } \\
\text { density }\left(\mathrm{A} \mathrm{cm}^{-2}\right)\end{array}$} & \multirow{2}{*}{$\begin{array}{c}\text { Mass } \\
\text { corrosion rate } \\
\left(\mathrm{g} \mathrm{cm}^{-2} \mathrm{~d}^{-1}\right)\end{array}$} & \multirow{2}{*}{$\begin{array}{l}\text { Penetration } \\
\text { rate }\left(\mathrm{mm} \mathrm{yr}^{-1}\right)\end{array}$} \\
\hline & & 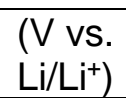 & $\begin{array}{c}\text { (V above } \\
\text { ECORR }_{\text {Con }}\end{array}$ & & & \\
\hline 1 & 500 & 1.64 & 0.2 & $1.8 \times 10^{-4}$ & $4.1 \times 10^{-3}$ & 1.9 \\
\hline 3 & 800 & 1.40 & 0.1 & $1.8 \times 10^{-4}$ & $4.1 \times 10^{-3}$ & 1.9 \\
\hline
\end{tabular}

\section{Molten salt splashing and aerosol formation}

The data provided in this section correspond to Section 6 in Thomas and Jackson, 2021, and the experimental design and methods are described therein. Table 31 provides a summary of the conditions of each FLiNaK splashing and aerosol generation test that was conducted. The conditions include the initial salt temperature; the average pour rate; the total mass of CsF, CsI, and FLiNaK added to the crucible prior to heating; and the mass that was poured into the spill containment box.

Table 31: Summary of splashing and aerosol generation test conditions for tests using molten FLiNaK with surrogate fission products ${ }^{a}$

\begin{tabular}{ccccccc}
\hline Test & $\begin{array}{c}\text { Initial salt temp. } \\
\left({ }^{\circ} \mathrm{C}\right) \text { estimate }^{\mathrm{b}}\end{array}$ & $\begin{array}{c}\text { Avg. pour } \\
\text { rate }\left(\mathrm{g} \mathrm{s}^{-1}\right)\end{array}$ & $\begin{array}{c}\text { Mass } \\
\text { CsF }(\mathrm{g})\end{array}$ & $\begin{array}{c}\text { Mass } \\
\text { Csl }(\mathrm{g})\end{array}$ & $\begin{array}{c}\text { Mass } \\
\text { FLiNaK }(\mathrm{g})\end{array}$ & $\begin{array}{c}\text { Mass } \\
\text { poured }(\mathrm{g})^{\mathrm{c}}\end{array}$ \\
\hline 1 & 500 & 18.4 & 0.51 & 0.10 & 14.45 & 14.15 \\
2 & 500 & 14.8 & 0.50 & 0.11 & 14.48 & 13.81 \\
3 & 650 & 19.6 & 0.48 & 0.09 & 14.44 & 14.52 \\
4 & 650 & 19.9 & 0.50 & 0.10 & 14.44 & 13.96 \\
5 & 800 & 21.2 & 0.49 & 0.11 & 14.45 & 14.66 \\
6 & 800 & 15.5 & 0.51 & 0.11 & 14.43 & 14.74 \\
7 & 800 & 16.9 & - & - & 15.08 & 14.86 \\
\hline
\end{tabular}

a The pour height was 15 in. for all tests.

$\mathrm{b}$ The initial salt temperature was estimated by a thermocouple placed in the furnace liner in which the salt was heated.

c The mass poured is equal to the mass of salt added to the crucible minus the mass of salt that remained in the crucible after pouring. 


\subsection{Video of splashing and mist formation}

Visible video of molten salt pouring and splashing into the spill containment box was filmed through a view port on the ceiling of the box using a visible camera that was mounted on a tripod. The visible video was filmed at 240 frames per second and real time and slow motion (20x slowed down) video of the splashing is available upon reasonable request. Visible video of mist floating above the molten salt pool in the spill containment box is also available for some tests. A list of available videos is provided in Table 32.

Table 32: List of available visible video of molten salt splashing tests

\begin{tabular}{|c|c|c|}
\hline Test & Video Description & Filename \\
\hline \multirow{2}{*}{1} & Splash video - real time & SplashingVideo_Test1.mp4 \\
\hline & Splash video $-20 x$ slow & SplashingVideo_Test1_slowmo.mp4 \\
\hline \multirow{2}{*}{2} & Splash video - real time & SplashingVideo_Test2.mp4 \\
\hline & Splash video - 20x slow & SplashingVideo_Test2_slowmo.mp4 \\
\hline \multirow{3}{*}{3} & Splash video - real time & SplashingVideo_Test3.mp4 \\
\hline & Splash video $-20 x$ slow & SplashingVideo_Test3_slowmo.mp4 \\
\hline & Mist video - real time & SplashingVideo_Test3_mist.mp4 \\
\hline \multirow{2}{*}{4} & Splash video - real time & SplashingVideo_Test4.mp4 \\
\hline & Splash video $-20 x$ slow & SplashingVideo_Test4_slowmo.mp4 \\
\hline \multirow{3}{*}{5} & Splash video - real time & SplashingVideo_Test5.mp4 \\
\hline & Splash video - 20x slow & SplashingVideo_Test5_slowmo.mp4 \\
\hline & Mist video - real time & SplashingVideo_Test5_mist.mp4 \\
\hline \multirow{3}{*}{6} & Splash video - real time & SplashingVideo_Test6.mp4 \\
\hline & Splash video $-20 x$ slow & SplashingVideo_Test6_slowmo.mp4 \\
\hline & Mist video - real time & SplashingVideo_Test6_mist.mp4 \\
\hline \multirow{2}{*}{7} & Splash video - real time & SplashingVideo_Test7.mp4 \\
\hline & Splash video - 20x slow & SplashingVideo_Test7_slowmo.mp4 \\
\hline
\end{tabular}

\subsection{Particle abundance and size distribution on coupons}

Coupons with dimensions of 1 in. $\times 1$ in. were hung on the walls of the catch pan surrounding the spill zone and on the wall furthest from the spill zone just below the aerosol collection filter to collect splatter for the determination of particle abundance and size distribution. The locations and labels of the coupons are shown in Figure 4. 


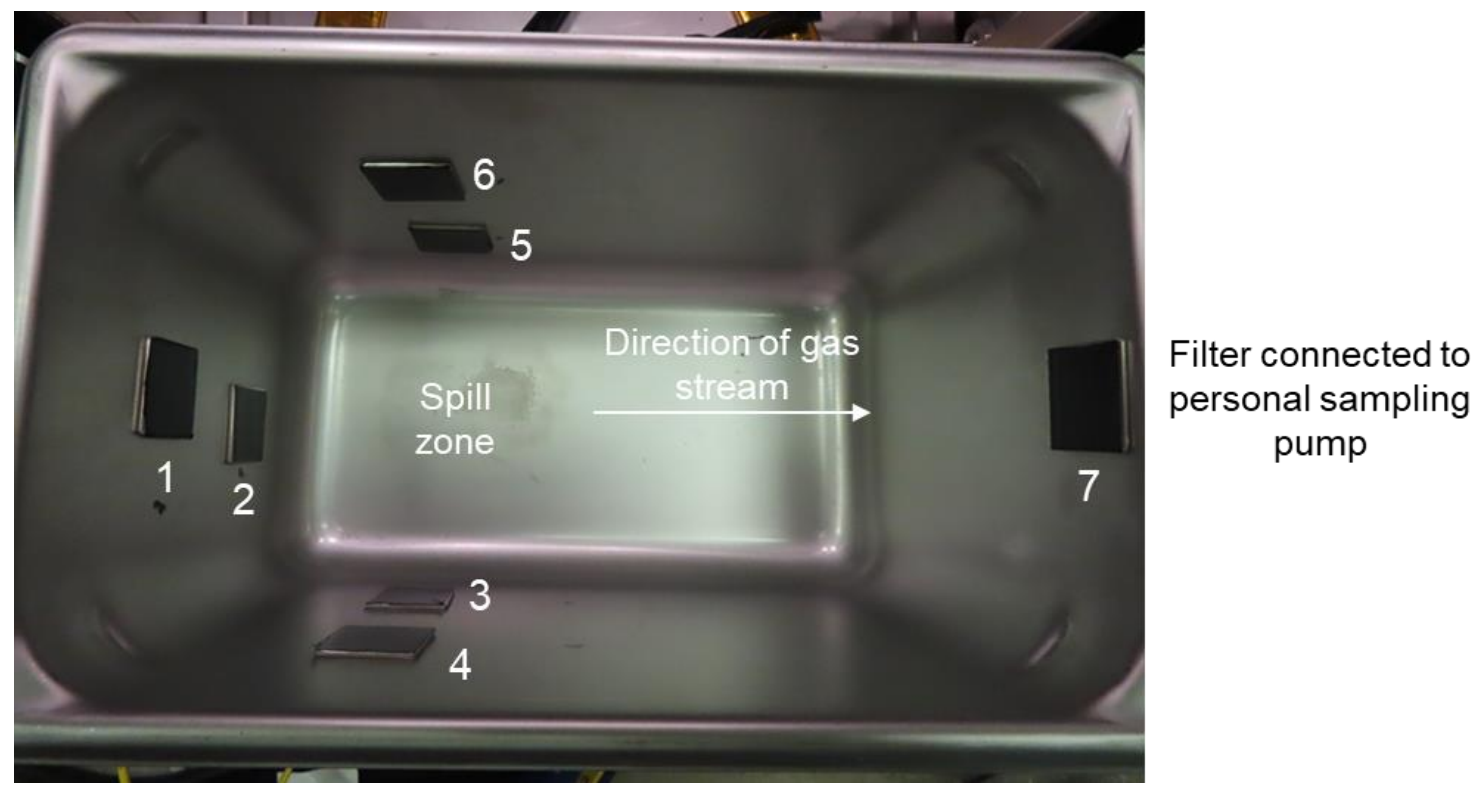

Figure 4: The coupon labels and locations in the catch pan relative to the salt spill zone. The filter for aerosol collection is positioned directly above Coupon 7 on the far wall from the spill zone.

Raw visible images of the coupons collected after the splash tests are available upon reasonable request. The splatter particle areas (2-dimensional projection) were determined from the processed images for each coupon as described in Section 6.1.2.7 in Thomas and Jackson, 2021 and the processed images and particle size data are available upon reasonable request. The total area analyzed for each coupon of each test is provided in Table 33.

Table 33: Summary of total area $\left(\mathrm{mm}^{2}\right)$ analyzed per coupon for each test

\begin{tabular}{cccccccc}
\hline & \multicolumn{7}{c}{ Coupon } \\
Test & 1 & 2 & 3 & 4 & 5 & 6 & 7 \\
\hline 1 & 424.5 & 332.0 & 399.7 & 158.8 & 295.5 & 516.2 & 392.9 \\
2 & 468.3 & 312.1 & 410.9 & 385.4 & 436.2 & 445.2 & 412.0 \\
3 & 514.8 & 342.6 & 457.5 & 469.1 & 558.6 & 168.7 & 354.7 \\
4 & - & - & 355.0 & 421.6 & 487.9 & 454.1 & 337.3 \\
5 & 552.2 & 503.3 & 491.7 & 405.8 & 360.0 & 494.7 & 445.1 \\
6 & 155.7 & 147.7 & 474.3 & 377.7 & 382.6 & 387.9 & 536.4 \\
7 & 286.0 & 567.6 & 574.3 & 407.2 & 573.6 & 451.1 & 426.9 \\
\hline
\end{tabular}

\subsection{Splatter quantification by mass fraction}

The total mass of salt, the mass of salt that formed a pool, and the mass of salt that formed splatter that was recovered from the catch pan after freezing is provided in Table 34 . The mass 
fraction of salt that was not recovered was determined from the difference between the mass of salt poured and the total mass of salt that was recovered.

Table 34: Mass balance results of salt recovered from catch pan after freezing

\begin{tabular}{ccccc}
\hline Test & $\begin{array}{c}\text { Total mass } \\
\text { recovered }(\mathrm{g})\end{array}$ & $\begin{array}{c}\text { Mass of } \\
\text { pooled salt }(\mathrm{g})\end{array}$ & $\begin{array}{c}\text { Mass of } \\
\text { splatter }(\mathrm{g})\end{array}$ & $\begin{array}{c}\text { Mass not } \\
\text { recovered }(\mathrm{g})\end{array}$ \\
\hline 1 & 13.98 & 10.84 & 3.13 & 0.17 \\
2 & 13.61 & 10.67 & 2.94 & 0.20 \\
3 & 14.25 & 11.48 & 2.77 & 0.27 \\
4 & 13.78 & 11.15 & 2.63 & 0.19 \\
5 & 14.36 & 11.75 & 2.61 & 0.30 \\
6 & 14.45 & 12.10 & 2.35 & 0.29 \\
7 & 14.59 & 12.03 & 2.56 & 0.27 \\
\hline
\end{tabular}

\subsection{Aerosol collection on filters}

Aerosol cassettes with 0.45 - $\mu$ m-pore size PTFE filters were used to sample the spill containment box atmosphere for suspended particulates using the methods described in Section 6.1.2.6 in Thomas and Jackson, 2021. The composition of collected particulates on the filters was analyzed as described in Section 2.3 of Thomas and Jackson, 2021 and the results are provided in Table 35. The filters were rinsed a second time with acid and the acid leachate was analyzed for total lithium, sodium, potassium, and cesium (Table 36).

Table 35: Elemental composition of particulates collected on $0.45-\mu \mathrm{m}$ filters measured in water leachate ${ }^{\mathrm{a}, \mathrm{b}}$

\begin{tabular}{ccccccccc}
\hline Test $^{\mathrm{c}}$ & $\begin{array}{c}\text { Target initial } \\
\text { temp. }\left({ }^{\circ} \mathrm{C}\right)\end{array}$ & $\begin{array}{c}\text { Sampling } \\
\text { time }(\mathrm{sec})\end{array}$ & $\begin{array}{c}\text { CsF \& CsI } \\
\text { in salt? }\end{array}$ & $\begin{array}{c}\mathrm{Li} \\
(\mu \mathrm{g})\end{array}$ & $\begin{array}{c}\mathrm{Na} \\
(\mu \mathrm{g})\end{array}$ & $\begin{array}{c}\mathrm{K} \\
(\mu \mathrm{g})\end{array}$ & $\begin{array}{c}\mathrm{Cs} \\
(\mu \mathrm{g})\end{array}$ & $\begin{array}{c}\mathrm{I} \\
(\mu \mathrm{g})\end{array}$ \\
\hline Blank $^{\mathrm{d}}$ & $\mathrm{n} / \mathrm{a}$ & 561 & $\mathrm{n} / \mathrm{a}$ & $<0.12$ & 0.63 & $<0.48$ & $<0.002$ & $<0.02$ \\
Control $^{\mathrm{e}}$ & $\mathrm{n} / \mathrm{a}$ & 553 & $\mathrm{n} / \mathrm{a}$ & $<0.01$ & 1.74 & $<0.55$ & 0.01 & $<0.03$ \\
1 & 500 & 573 & Yes & $<0.01$ & 1.30 & 0.65 & 0.12 & $<0.03$ \\
2 & 500 & 558 & Yes & $<0.12$ & 40.5 & $<0.48$ & 0.14 & $<0.02$ \\
3 & 650 & 524 & Yes & $<0.01$ & 2.29 & 1.97 & 0.41 & $<0.03$ \\
4 & 650 & 561 & Yes & $<0.12$ & 0.88 & $<0.48$ & 0.33 & $<0.05$ \\
5 & 800 & 553 & Yes & $<0.01$ & 1.45 & 1.44 & 1.76 & 0.15 \\
7 & 800 & 540 & No & $<0.01$ & 1.82 & 1.40 & 0.01 & $<0.03$ \\
\hline
\end{tabular}

a The number following the $<$ is the detection limit.

$\mathrm{b}$ The total mass of particulates collected on the filter was too small to measure.

${ }^{\mathrm{c}}$ A filter measurement was not conducted for Test 6 at an initial salt target temperature of $800{ }^{\circ} \mathrm{C}$ because aerosols were collected using cascade impactors instead.

d The composition of the blank filter was measured directly after removal from its packaging.

e The control filter sampled the background glovebox atmosphere for 10 minutes before any

FLiNaK splash tests were conducted. 
Table 36: Elemental composition of particulates collected on $0.45-\mu \mathrm{m}$ filters after acid leach ${ }^{\mathrm{a}, \mathrm{b}}$

\begin{tabular}{ccccccc}
\hline Test $^{\mathrm{c}}$ & $\begin{array}{c}\text { Target initial } \\
\text { temp. }\left({ }^{\circ} \mathrm{C}\right)\end{array}$ & $\begin{array}{c}\text { CsF \& CsI } \\
\text { in salt? }\end{array}$ & $\begin{array}{c}\mathrm{Li} \\
(\mu \mathrm{g})\end{array}$ & $\begin{array}{c}\mathrm{Na} \\
(\mu \mathrm{g})\end{array}$ & $\begin{array}{c}\mathrm{K} \\
(\mu \mathrm{g})\end{array}$ & $\begin{array}{c}\mathrm{Cs} \\
(\mu \mathrm{g})\end{array}$ \\
\hline Blank & $\mathrm{n} / \mathrm{a}$ & $\mathrm{n} / \mathrm{a}$ & 0.29 & 0.21 & $<1.96$ & $<0.004$ \\
Control & $\mathrm{n} / \mathrm{a}$ & $\mathrm{n} / \mathrm{a}$ & 0.14 & 0.07 & $<1.96$ & 0.01 \\
1 & 500 & Yes & 0.13 & 0.12 & $<1.96$ & $<0.008$ \\
2 & 500 & Yes & 0.22 & 0.10 & $<1.96$ & 0.03 \\
3 & 650 & Yes & 0.22 & 0.11 & $<1.96$ & 0.05 \\
4 & 650 & Yes & 0.16 & 0.07 & $<1.96$ & 0.02 \\
5 & 800 & Yes & 0.14 & 0.04 & $<1.96$ & 0.02 \\
7 & 800 & No & 0.13 & 0.07 & $<1.96$ & $<0.004$ \\
\hline
\end{tabular}

${ }^{a}$ The number following the $<$ is the detection limit.

${ }^{\mathrm{b}}$ lodine was not quantified in the acid leachate solution.

${ }^{\mathrm{C}} \mathrm{A}$ filter measurement was not conducted for Test 6 at an initial salt target temperature of $800{ }^{\circ} \mathrm{C}$ because aerosols were collected using cascade impactors instead.

\subsection{Temperatures measured in the spill containment box}

The temperature of the catch pan underside beneath the salt spill zone (see Figure 4) is provided as a function of time after spilling the salt for each test in Table 37.

Table 37: Temp. $\left({ }^{\circ} \mathrm{C}\right)$ measured by thermocouple attached to underside of stainless steel catch pan

\begin{tabular}{cccccccc}
\hline Time $(\min )$. & Test 1 & Test 2 & Test 3 & Test 4 & Test 5 & Test 6 & Test 7 \\
\hline 0.00 & 26.5 & 25.2 & 26.4 & 25.1 & 29.2 & 25.0 & 27.8 \\
0.25 & 54.4 & 37.6 & 90.9 & 71.2 & 163.1 & 147.2 & 135.9 \\
0.50 & 80.7 & 53.7 & 150.3 & 113.6 & 241.4 & 234.5 & 209.0 \\
0.75 & 100.2 & 68.2 & 191.9 & 140.9 & 272.0 & 287.1 & 251.3 \\
1.00 & 113.0 & 79.6 & 218.8 & 156.8 & 280.5 & 309.8 & 273.9 \\
1.25 & 120.2 & 87.5 & 233.6 & 165.6 & 278.4 & 312.2 & 284.1 \\
1.50 & 123.2 & 92.7 & 239.9 & 169.8 & 270.7 & 305.3 & 286.1 \\
1.75 & 123.4 & 95.8 & 240.8 & 171.0 & 260.3 & 293.5 & 282.2 \\
2.00 & 122.2 & 97.4 & 237.8 & 170.0 & 248.7 & 279.9 & 274.6 \\
2.25 & 120.1 & 97.8 & 232.2 & 167.4 & 236.9 & 265.6 & 265.1 \\
2.50 & 117.4 & 97.4 & 224.7 & 163.6 & 225.2 & 251.3 & 254.5 \\
2.75 & 114.4 & 96.4 & 216.3 & 158.9 & 213.9 & 237.6 & 243.4 \\
3.00 & 111.3 & 95.0 & 207.4 & 153.8 & 203.0 & 224.6 & 232.5 \\
3.25 & 108.1 & 93.2 & 198.2 & 148.3 & 192.7 & 212.3 & 221.8 \\
3.50 & 104.9 & 91.2 & 189.1 & 142.7 & 183.0 & 200.8 & 211.4 \\
3.75 & 101.7 & 89.0 & 180.3 & 137.0 & 173.9 & 190.1 & 201.5 \\
4.00 & 98.5 & 86.8 & 171.9 & 131.4 & 165.4 & 180.2 & 192.0 \\
4.25 & 95.4 & 84.6 & 163.8 & 125.9 & 157.6 & 171.0 & 183.1 \\
4.50 & 92.3 & 82.4 & 156.1 & 120.6 & 150.2 & 162.4 & 174.7 \\
4.75 & 89.4 & 80.2 & 148.9 & 115.5 & 143.3 & 154.5 & 166.7 \\
5.00 & 86.6 & 77.9 & 141.9 & 110.7 & 136.8 & 147.0 & 159.2 \\
\hline
\end{tabular}


Table 24: (cont.)

\begin{tabular}{cccccccc}
\hline Time $(\min )$. & Test 1 & Test 2 & Test 3 & Test 4 & Test 5 & Test 6 & Test 7 \\
\hline 5.25 & 83.9 & 75.8 & 135.2 & 106.1 & 130.8 & 140.0 & 152.3 \\
5.50 & 81.3 & 73.7 & 128.8 & 101.8 & 125.1 & 133.5 & 145.7 \\
5.75 & 78.9 & 71.7 & 123.0 & 97.7 & 119.9 & 127.4 & 139.5 \\
6.00 & 76.5 & 69.7 & 117.4 & 93.8 & 114.9 & 121.7 & 133.7 \\
6.25 & 74.2 & 67.8 & 112.3 & 90.2 & 110.3 & 116.4 & 128.2 \\
6.50 & 72.1 & 66.0 & 107.5 & 86.8 & 106.0 & 111.5 & 123.0 \\
6.75 & 69.9 & 64.2 & 103.0 & 83.5 & 102.1 & 106.8 & 118.2 \\
7.00 & 67.9 & 62.5 & 98.9 & 80.5 & 98.3 & 102.5 & 113.7 \\
7.25 & 66.0 & 60.9 & 95.0 & 77.5 & 94.6 & 98.5 & 109.3 \\
7.50 & 64.2 & 59.4 & 91.3 & 74.7 & 91.3 & 94.7 & 105.3 \\
7.75 & 62.5 & 57.9 & 87.9 & 72.1 & 88.2 & 91.1 & 101.5 \\
8.00 & 60.8 & 56.5 & 84.7 & 69.6 & 85.2 & 87.8 & 97.9 \\
8.25 & 59.3 & 55.1 & 81.6 & 67.2 & 82.4 & 84.6 & 94.6 \\
8.50 & 57.8 & 53.8 & 78.8 & 65.0 & 79.8 & 81.7 & 91.4 \\
8.75 & 56.4 & 52.6 & 76.2 & 62.9 & 77.4 & 79.0 & 88.4 \\
9.00 & 55.1 & 51.5 & 73.7 & 61.0 & 75.0 & 76.4 & 85.6 \\
9.25 & 53.8 & 50.3 & 71.4 & 59.2 & 72.9 & 73.9 & 83.0 \\
9.50 & 52.6 & 49.3 & 69.1 & 57.5 & 70.8 & 71.6 & 80.4 \\
9.75 & 51.5 & 48.2 & 67.1 & 55.9 & 68.9 & 69.5 & 78.0 \\
10.00 & 50.4 & 47.3 & 65.1 & 54.4 & 67.0 & 67.4 & 75.8 \\
\hline
\end{tabular}

The temperatures of various locations within the spill containment box atmosphere are provided as a function of time after spilling the salt in Table 38, Table 39, Table 40, Table 41, Table 42, Table 43, and Table 44 for Tests $1,2,3,4,5,6$, and 7, respectively. The locations of thermocouples TC2, TC3, and TC4 measuring the temperatures of the spill containment box atmosphere are shown in Figure 5.

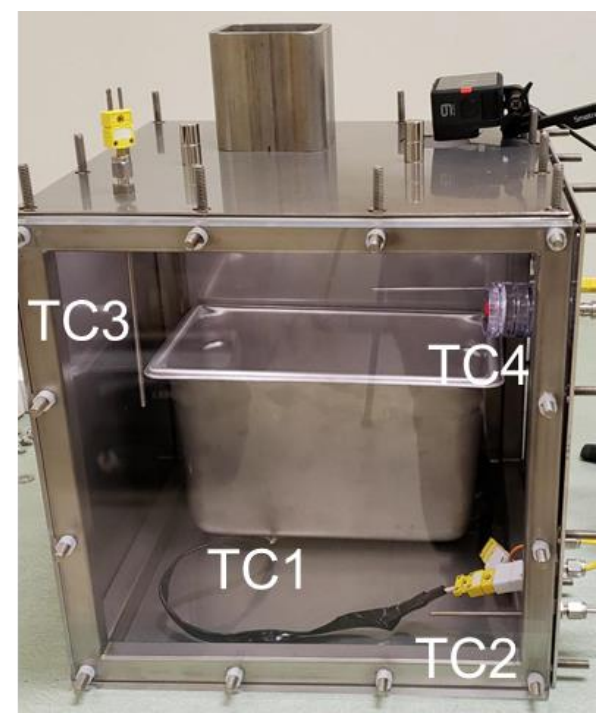

Figure 5: The locations of thermocouples TC2, TC3, and TC4 that measured the temperature of the spill containment box atmosphere. TC1 is the thermocouple that measured the temperature of the catch pan underside. 
Table 38: The temp. $\left({ }^{\circ} \mathrm{C}\right)$ of the spill containment box atmosphere by thermocouple for Test 1

\begin{tabular}{cccc}
\hline Time (min.) & TC2 & TC3 & TC4 \\
\hline 0.0 & 28.0 & 29.7 & 27.2 \\
0.5 & 28.2 & 30.1 & 28.6 \\
1.0 & 28.5 & 30.6 & 29.8 \\
1.5 & 28.7 & 31.0 & 30.5 \\
2.0 & 28.9 & 31.4 & 31.0 \\
2.5 & 29.1 & 31.7 & 31.3 \\
3.0 & 29.2 & 32.0 & 31.5 \\
3.5 & 29.4 & 32.2 & 31.5 \\
4.0 & 29.5 & 32.4 & 31.5 \\
4.5 & 29.5 & 32.5 & 31.5 \\
5.0 & 29.6 & 32.7 & 31.4 \\
5.5 & 29.6 & 32.8 & 31.3 \\
6.0 & 29.7 & 32.9 & 31.2 \\
6.5 & 29.7 & 33.0 & 31.1 \\
7.0 & 29.7 & 33.0 & 31.0 \\
7.5 & 29.7 & 33.1 & 30.8 \\
8.0 & 29.7 & 33.1 & 30.7 \\
8.5 & 29.8 & 33.2 & 30.6 \\
9.0 & 29.8 & 33.2 & 30.5 \\
9.5 & 29.7 & 33.2 & 30.4 \\
10.0 & 29.7 & 33.2 & 30.2 \\
\hline
\end{tabular}

Table 39: The temp. $\left({ }^{\circ} \mathrm{C}\right)$ of the spill containment box atmosphere by thermocouple for Test 2

\begin{tabular}{cccc}
\hline Time $(\min )$. & TC2 & TC3 & TC4 \\
\hline 0.0 & 26.6 & 28.6 & 26.0 \\
0.5 & 26.7 & 29.5 & 27.6 \\
1.0 & 26.9 & 30.1 & 28.6 \\
1.5 & 27.1 & 30.4 & 29.1 \\
2.0 & 27.3 & 30.7 & 29.2 \\
2.5 & 27.4 & 31.0 & 29.3 \\
3.0 & 27.5 & 31.2 & 29.2 \\
3.5 & 27.7 & 31.3 & 29.1 \\
4.0 & 27.8 & 31.5 & 29.0 \\
4.5 & 27.9 & 31.6 & 28.9 \\
5.0 & 28.0 & 31.8 & 28.8 \\
5.5 & 28.1 & 31.8 & 28.7 \\
6.0 & 28.1 & 31.9 & 28.6 \\
6.5 & 28.2 & 31.9 & 28.6 \\
7.0 & 28.2 & 31.9 & 28.5 \\
7.5 & 28.3 & 32.0 & 28.4 \\
8.0 & 28.3 & 31.9 & 28.3 \\
8.5 & 28.3 & 31.9 & 28.3 \\
9.0 & 28.4 & 31.9 & 28.2 \\
9.5 & 28.4 & 31.9 & 28.1 \\
10.0 & 28.3 & 31.9 & 28.1 \\
\hline
\end{tabular}


Table 40: The temp. $\left({ }^{\circ} \mathrm{C}\right)$ of the spill containment box atmosphere by thermocouple for Test 3

\begin{tabular}{cccc}
\hline Time (min.) & TC2 & TC3 & TC4 \\
\hline 0.0 & 27.6 & 29.7 & 27.0 \\
0.5 & 27.7 & 30.2 & 28.2 \\
1.0 & 27.9 & 30.7 & 29.4 \\
1.5 & 28.1 & 31.3 & 30.3 \\
2.0 & 28.3 & 31.7 & 30.9 \\
2.5 & 28.5 & 32.1 & 31.3 \\
3.0 & 28.6 & 32.4 & 31.5 \\
3.5 & 28.7 & 32.7 & 31.7 \\
4.0 & 28.8 & 32.9 & 31.7 \\
4.5 & 28.9 & 33.1 & 31.7 \\
5.0 & 29.0 & 33.3 & 31.7 \\
5.5 & 29.1 & 33.5 & 31.6 \\
6.0 & 29.2 & 33.6 & 31.5 \\
6.5 & 29.3 & 33.7 & 31.4 \\
7.0 & 29.4 & 33.8 & 31.3 \\
7.5 & 29.4 & 33.8 & 31.1 \\
8.0 & 29.5 & 33.9 & 31.0 \\
8.5 & 29.5 & 33.9 & 31.0 \\
9.0 & 29.5 & 33.9 & 30.9 \\
9.5 & 29.6 & 33.9 & 30.8 \\
10.0 & 29.6 & 33.9 & 30.7 \\
\hline
\end{tabular}

Table 41: The temp. $\left({ }^{\circ} \mathrm{C}\right)$ of the spill containment box atmosphere by thermocouple for Test 4

\begin{tabular}{cccc}
\hline Time $(\min )$. & TC2 & TC3 & TC4 \\
\hline 0.0 & 26.5 & 28.8 & 25.9 \\
0.5 & 26.7 & 29.4 & 27.6 \\
1.0 & 27.0 & 30.1 & 28.9 \\
1.5 & 27.2 & 30.6 & 29.7 \\
2.0 & 27.4 & 31.1 & 30.1 \\
2.5 & 27.6 & 31.5 & 30.2 \\
3.0 & 27.8 & 31.8 & 30.2 \\
3.5 & 27.9 & 32.2 & 30.2 \\
4.0 & 28.0 & 32.4 & 30.1 \\
4.5 & 28.2 & 32.6 & 30.0 \\
5.0 & 28.3 & 32.8 & 30.0 \\
5.5 & 28.3 & 32.9 & 29.9 \\
6.0 & 28.4 & 33.0 & 29.8 \\
6.5 & 28.5 & 33.1 & 29.7 \\
7.0 & 28.5 & 33.1 & 29.6 \\
7.5 & 28.6 & 33.2 & 29.5 \\
8.0 & 28.6 & 33.2 & 29.4 \\
8.5 & 28.6 & 33.2 & 29.3 \\
9.0 & 28.6 & 33.2 & 29.3 \\
9.5 & 28.7 & 33.2 & 29.2 \\
10.0 & 28.7 & 33.1 & 29.1 \\
\hline
\end{tabular}


Table 42: The temp. $\left({ }^{\circ} \mathrm{C}\right)$ of the spill containment box atmosphere by thermocouple for Test 5

\begin{tabular}{cccc}
\hline Time (min.) & TC2 & TC3 & TC4 \\
\hline 0.0 & 29.2 & 31.6 & 28.8 \\
0.5 & 29.5 & 32.2 & 30.6 \\
1.0 & 29.8 & 32.8 & 31.8 \\
1.5 & 30.1 & 33.4 & 32.8 \\
2.0 & 30.4 & 34.0 & 33.5 \\
2.5 & 30.6 & 34.5 & 33.9 \\
3.0 & 30.8 & 35.0 & 34.2 \\
3.5 & 31.0 & 35.4 & 34.3 \\
4.0 & 31.1 & 35.7 & 34.3 \\
4.5 & 31.3 & 36.0 & 34.3 \\
5.0 & 31.4 & 36.3 & 34.2 \\
5.5 & 31.4 & 36.5 & 34.1 \\
6.0 & 31.5 & 36.7 & 34.0 \\
6.5 & 31.6 & 36.8 & 33.8 \\
7.0 & 31.7 & 37.0 & 33.8 \\
7.5 & 31.7 & 37.0 & 33.6 \\
8.0 & 31.7 & 37.1 & 33.5 \\
8.5 & 31.7 & 37.1 & 33.4 \\
9.0 & 31.8 & 37.1 & 33.3 \\
9.5 & 31.8 & 37.2 & 33.2 \\
10.0 & 31.8 & 37.2 & 33.1 \\
\hline
\end{tabular}

Table 43: The temp. $\left({ }^{\circ} \mathrm{C}\right)$ of the spill containment box atmosphere by thermocouple for Test 6

\begin{tabular}{cccc}
\hline Time $($ min.) & TC2 & TC3 & TC4 \\
\hline 0.0 & 26.3 & 28.5 & 25.8 \\
0.5 & 26.6 & 29.0 & 27.5 \\
1.0 & 26.9 & 29.6 & 29.0 \\
1.5 & 27.2 & 30.1 & 30.1 \\
2.0 & 27.5 & 30.7 & 30.9 \\
2.5 & 27.8 & 31.1 & 31.4 \\
3.0 & 28.0 & 31.5 & 31.6 \\
3.5 & 28.3 & 31.9 & 31.8 \\
4.0 & 28.5 & 32.2 & 31.8 \\
4.5 & 28.6 & 32.4 & 31.8 \\
5.0 & 28.8 & 32.7 & 31.8 \\
5.5 & 28.9 & 32.8 & 31.7 \\
6.0 & 29.0 & 33.0 & 31.6 \\
6.5 & 29.1 & 33.1 & 31.5 \\
7.0 & 29.3 & 33.2 & 31.4 \\
7.5 & 29.3 & 33.3 & 31.3 \\
8.0 & 29.4 & 33.3 & 31.2 \\
8.5 & 29.4 & 33.4 & 31.1 \\
9.0 & 29.5 & 33.5 & 31.0 \\
9.5 & 29.5 & 33.4 & 30.9 \\
10.0 & 29.6 & 33.5 & 30.8 \\
\hline
\end{tabular}


Table 44: The temp. $\left({ }^{\circ} \mathrm{C}\right)$ of the spill containment box atmosphere by thermocouple for Test 7

\begin{tabular}{cccc}
\hline Time $(\min )$. & TC2 & TC3 & TC4 \\
\hline 0.0 & 29.3 & 31.5 & 29.0 \\
0.5 & 29.6 & 32.2 & 30.8 \\
1.0 & 29.9 & 33.0 & 32.0 \\
1.5 & 30.2 & 33.7 & 32.7 \\
2.0 & 30.4 & 34.3 & 33.1 \\
2.5 & 30.7 & 34.8 & 33.4 \\
3.0 & 30.9 & 35.3 & 33.5 \\
3.5 & 31.1 & 35.8 & 33.6 \\
4.0 & 31.3 & 36.2 & 33.6 \\
4.5 & 31.4 & 36.5 & 33.6 \\
5.0 & 31.5 & 36.8 & 33.5 \\
5.5 & 31.6 & 37.0 & 33.4 \\
6.0 & 31.7 & 37.1 & 33.4 \\
6.5 & 31.8 & 37.3 & 33.3 \\
7.0 & 31.9 & 37.4 & 33.2 \\
7.5 & 31.9 & 37.5 & 33.1 \\
8.0 & 32.0 & 37.5 & 33.1 \\
8.5 & 32.0 & 37.5 & 33.0 \\
9.0 & 32.0 & 37.6 & 32.9 \\
9.5 & 32.1 & 37.6 & 32.9 \\
10.0 & 32.1 & 37.6 & 32.8 \\
\hline
\end{tabular}

\subsection{Glovebox conditions}

The $\mathrm{O}_{2}$ content and $\mathrm{H}_{2} \mathrm{O}$ content of the argon atmosphere glovebox was recorded before and after each test and is provided in Table 45. The temperature of various locations within the spill containment box atmosphere was recorded throughout the duration of the tests and is discussed in Section 5.5.

Table 45: The $\mathrm{O}_{2}$ content and $\mathrm{H}_{2} \mathrm{O}$ content of the glovebox atmosphere measured before and after each test

\begin{tabular}{ccccc}
\hline & \multicolumn{2}{c}{$\mathrm{O}_{2}$ content $(\mathrm{ppm})$} & \multicolumn{2}{c}{$\mathrm{H}_{2} \mathrm{O}$ content $(\mathrm{ppm})$} \\
\cline { 2 - 5 } Test & Before & After & Before & After \\
\hline 1 & $<1$ & $<1$ & 5 & 5 \\
2 & $<1$ & $<1$ & 4 & 4 \\
3 & 28 & 28 & $<1$ & $<1$ \\
4 & $<1$ & $<1$ & 1.5 & 1.5 \\
5 & $<1$ & $<1$ & 4 & 4 \\
6 & 1 & 1 & $<1$ & $<1$ \\
7 & 1.5 & 1.5 & 2 & 2 \\
\hline
\end{tabular}




\subsection{Salt composition analysis by ICP-MS}

Table 46 provides the concentration of major cations (lithium, sodium, potassium, and cesium) and iodine and Table 47 provides the concentration of trace metals that were measured in the salt after splashing for each test. The samples from each test were taken from the salt that pooled at the bottom of the catch pan (pool) and the splatter particles that dispersed within the catch pan (splatter). The measurement methods are described in Section 2.3 in Thomas and Jackson, 2021.

Table 46: Composition of frozen salt samples collected from splash tests ${ }^{a}$

\begin{tabular}{|c|c|c|c|c|c|c|c|c|}
\hline Test & $\begin{array}{c}\text { Target } \\
\text { initial temp. } \\
\left({ }^{\circ} \mathrm{C}\right)\end{array}$ & $\begin{array}{c}\text { CsF \& Csl } \\
\text { in salt? }\end{array}$ & $\begin{array}{c}\text { Sample } \\
\text { type }\end{array}$ & $\begin{array}{c}\mathrm{LiF} \\
(\mathrm{mol} \%)\end{array}$ & $\begin{array}{c}\mathrm{NaF} \\
(\mathrm{mol} \%)\end{array}$ & $\begin{array}{c}\mathrm{KF} \\
(\mathrm{mol} \%)\end{array}$ & $\begin{array}{c}\mathrm{CsF} \\
(\mathrm{mol} \%)\end{array}$ & $\begin{array}{c}\mathrm{Cs}^{\mathrm{a}} \\
(\mathrm{mol} \%)\end{array}$ \\
\hline \multirow{2}{*}{1} & \multirow{2}{*}{500} & \multirow{2}{*}{ Yes } & Splatter & 48.40 & 11.27 & 39.28 & 0.97 & 0.09 \\
\hline & & & Pool & 48.63 & 11.26 & 39.12 & 0.91 & 0.08 \\
\hline \multirow{2}{*}{2} & \multirow{2}{*}{500} & \multirow{2}{*}{ Yes } & Splatter & 44.94 & 11.55 & 42.58 & 0.84 & 0.10 \\
\hline & & & Pool & 46.39 & 11.48 & 41.25 & 0.79 & 0.08 \\
\hline \multirow{2}{*}{3} & \multirow{2}{*}{650} & \multirow{2}{*}{ Yes } & Splatter & 47.72 & 11.54 & 39.67 & 0.98 & 0.09 \\
\hline & & & Pool & 48.49 & 11.32 & 39.18 & 0.92 & 0.09 \\
\hline \multirow{2}{*}{4} & \multirow{2}{*}{650} & \multirow{2}{*}{ Yes } & Splatter & 46.06 & 11.68 & 41.26 & 0.88 & 0.12 \\
\hline & & & Pool & 45.18 & 11.87 & 42.01 & 0.82 & 0.11 \\
\hline \multirow{2}{*}{5} & \multirow{2}{*}{800} & \multirow{2}{*}{ Yes } & Splatter & 47.22 & 11.72 & 40.05 & 0.89 & 0.12 \\
\hline & & & Pool & 48.78 & 11.25 & 38.97 & 0.88 & 0.12 \\
\hline \multirow{2}{*}{6} & \multirow{2}{*}{800} & \multirow{2}{*}{ Yes } & Splatter & 46.02 & 11.17 & 41.78 & 0.92 & 0.11 \\
\hline & & & Pool & 46.37 & 11.26 & 41.39 & 0.87 & 0.11 \\
\hline \multirow{2}{*}{7} & \multirow{2}{*}{800} & \multirow{2}{*}{ No } & Splatter & 50.6 & 11.0 & 38.5 & $\mathrm{n} / \mathrm{a}$ & $\mathrm{n} / \mathrm{a}$ \\
\hline & & & Pool & 49.3 & 11.3 & 39.4 & $\mathrm{n} / \mathrm{a}$ & $\mathrm{n} / \mathrm{a}$ \\
\hline
\end{tabular}

${ }^{a} \mathrm{Mol} \%$ of CsI was calculated assuming all detected iodine was present as Csl. 
Table 47: Trace metal composition of frozen salt samples collected from splash tests ${ }^{a, b}$

\begin{tabular}{|c|c|c|c|c|c|c|c|c|c|}
\hline Test & $\begin{array}{c}\text { Target } \\
\text { initial temp. } \\
\left({ }^{\circ} \mathrm{C}\right)\end{array}$ & $\begin{array}{l}\text { CsF \& } \\
\text { Csl in } \\
\text { salt? }\end{array}$ & $\begin{array}{c}\text { Sample } \\
\text { type }\end{array}$ & $\begin{array}{c}\mathrm{Ca} \\
\text { (ppm) }\end{array}$ & $\begin{array}{c}\mathrm{Cr} \\
(\mathrm{ppm})\end{array}$ & $\begin{array}{c}\mathrm{Mn} \\
(\mathrm{ppm})\end{array}$ & $\begin{array}{c}\mathrm{Ni} \\
(\mathrm{ppm})\end{array}$ & $\begin{array}{c}\mathrm{Rb} \\
(\mathrm{ppm})\end{array}$ & $\begin{array}{c}\mathrm{Cs}^{\mathrm{c}} \\
(\mathrm{ppm})\end{array}$ \\
\hline \multirow{2}{*}{1} & \multirow{2}{*}{500} & \multirow{2}{*}{ Yes } & Splatter & $<203$ & $<1.52$ & 1.31 & 41.7 & 24.6 & - \\
\hline & & & Pool & $<127$ & 13.3 & 1.57 & 28.7 & 25.0 & - \\
\hline \multirow{2}{*}{2} & \multirow{2}{*}{500} & \multirow{2}{*}{ Yes } & Splatter & 373 & $<1.27$ & $<1.30$ & 47.9 & 25.3 & - \\
\hline & & & Pool & 577 & $<1.37$ & 3.03 & 26.1 & 24.6 & - \\
\hline \multirow{2}{*}{3} & \multirow{2}{*}{650} & \multirow{2}{*}{ Yes } & Splatter & $<581$ & $<1.83$ & 2.77 & 177 & 24.6 & - \\
\hline & & & Pool & $<656$ & $<1.81$ & 6.87 & 8.05 & 24.7 & - \\
\hline \multirow{2}{*}{4} & \multirow{2}{*}{650} & \multirow{2}{*}{ Yes } & Splatter & 418 & 6.20 & 2.39 & 96.5 & 24.6 & - \\
\hline & & & Pool & 517 & $<1.19$ & 2.18 & 52.5 & 23.5 & - \\
\hline \multirow{2}{*}{5} & \multirow{2}{*}{800} & \multirow{2}{*}{ Yes } & Splatter & $<488$ & $<1.66$ & 32.9 & 24.3 & 23.3 & - \\
\hline & & & Pool & $<635$ & $<1.93$ & 4.49 & 26.1 & 24.3 & - \\
\hline \multirow{2}{*}{6} & \multirow{2}{*}{800} & \multirow{2}{*}{ Yes } & Splatter & $<186$ & 2.78 & 2.86 & 24.3 & 25.4 & - \\
\hline & & & Pool & 648 & 2.83 & 3.73 & 22.6 & 25.1 & - \\
\hline \multirow{2}{*}{7} & \multirow{2}{*}{800} & \multirow{2}{*}{ No } & Splatter & $<104$ & $<2.09$ & 4.50 & $<0.54$ & 25.4 & 38.9 \\
\hline & & & Pool & $<635$ & $<1.86$ & 6.08 & $<0.66$ & 23.8 & 13.2 \\
\hline
\end{tabular}

a The concentration of $\mathrm{Fe}$ in all samples was below the detection limit of approximately $70 \mathrm{ppm}$.

$\mathrm{b}$ The number after $<$ is the detection limit.

c The Cs concentration of FLiNaK doped with CsF and Csl is reported in Table 46.

\subsection{Composition of condensation on ceiling of spill containment box}

The ceiling of the spill containment box was wiped with a methanol-soaked glass fiber filter and analyzed for total lithium, sodium, potassium, cesium, and iodine by using ICP-MS as described in Section 6.2.6 in Thomas and Jackson, 2021. The results from the first leach of the wipe with water are provided in Table 48 and the results from the second leach of the wipe in acid are provided in Table 49. The fact that a significant amount of sodium and potassium are acid-leached from the glass fiber filters of the blank and control indicate that acid leaching is not compatible with the filter material.

Table 48: Elemental composition of wipe sample from ceiling of spill containment box measured after first leach with water ${ }^{\mathrm{a}}$

\begin{tabular}{cccccc}
\hline Test & $\mathrm{Li}(\mu \mathrm{g})$ & $\mathrm{Na}(\mu \mathrm{g})$ & $\mathrm{K}(\mu \mathrm{g})$ & $\mathrm{Cs}(\mu \mathrm{g})$ & $\mathrm{I}(\mu \mathrm{g})$ \\
\hline Blank $^{\mathrm{b}}$ & $<0.14$ & 166 & 7.6 & $<0.003$ & $<0.03$ \\
Control $^{\mathrm{c}}$ & $<0.16$ & 174 & 9.7 & 0.05 & 0.17 \\
Sample & 2.00 & 102 & 10.6 & 0.23 & 0.13
\end{tabular}

${ }^{a}$ The number following the $<$ is the detection limit.

${ }^{\mathrm{b}}$ The Blank was a wipe that was removed from its packaging and directly analyzed.

${ }^{c}$ The Control was a wipe that was soaked in methanol and then analyzed. 
Table 49: Elemental composition of wipe sample from ceiling of spill containment box measured after second leach with acida,b

\begin{tabular}{ccccc}
\hline Test & $\mathrm{Li}(\mu \mathrm{g})$ & $\mathrm{Na}(\mu \mathrm{g})$ & $\mathrm{K}(\mu \mathrm{g})$ & $\mathrm{Cs}(\mu \mathrm{g})$ \\
\hline Blank $^{\mathrm{c}}$ & 1.50 & 5760 & 2150 & 0.23 \\
Control $^{\mathrm{d}}$ & 1.67 & 5520 & 2120 & 2.51 \\
Sample & 1.50 & 2850 & 1090 & 4.18 \\
\hline
\end{tabular}

a The number following the $<$ is the detection limit.

${ }^{b}$ lodine was not quantified in the acid leachate solution.

$c$ The Blank was a wipe that was removed from its packaging and directly analyzed.

$d$ The Control was a wipe that was soaked in methanol and then analyzed.

\subsection{Splatter and aerosol particle composition by SEM-EDS}

Splatter that was collected on coupons was analyzed using scanning electron microscopy (SEM) with associated energy dispersive X-ray spectroscopy (EDS), and a description of select SEM micrographs, EDS emission maps, EDS line scans, and EDS spectra are described in Section 6.2.7 of Thomas and Jackson, 2021. Additional SEM micrographs and data from EDS analysis is available from the authors upon reasonable request. 


\section{References}

Lichtenstein, T., Rose, M. A., Krueger, J., Wu, E., and Williamson, M. A. (2020). "Thermochemical Property Measurements of FLiNaK and FLiBe in FY 2020." Argonne National Laboratory report. ANL/CFCT-20/37.

Thomas, S. and Jackson, J. (2021). "Testing to Evaluate Processes Expected to Occur during MSR Salt Spill Accidents." Argonne National Laboratory report. ANL/CFCT-21/22. 


\section{Argonne}

\section{Chemical and Fuel Cycle Technologies Division}

Argonne National Laboratory

9700 South Cass Avenue, Bldg. 205

Argonne, IL 60439

www.anl.gov 